\title{
Selected abstracts from the XXXVIII National Congress of the Italian Society of Hypertension (SIIA)
}

Metabolic Aspects and Associated Risk Factors

NEUROADRENEGIC ACTIVATION

IN OBSTRUCTIVE SLEEP APNEA SYNDROME: A SYSTEMATIC REVIEW AND META-ANALYSIS

Gino Seravalle ${ }^{1}$, Annalisa Biffi ${ }^{2}$, Matilde Bonzani ${ }^{3}$, Fosca Quarti Trevano ${ }^{4}$, Giovanni Corrao ${ }^{3}$, Giuseppe Mancia ${ }^{5}$, Guido Grassi ${ }^{4}$

${ }^{1}$ Cardiology Dept, IRCCS Italian Auxology Institute, Milan, Italy, ${ }^{2}$ Statistic Dept, University Milan-Bicocca, Milan, Italy, ${ }^{3}$ National Center for Health Research and Pharmaco-Epidemiology, Milan, Italy, ${ }^{4}$ Medicine Dept, University Milan-Bicocca, Monza, Italy, ${ }^{5}$ Policlinic of Monza and University Milan-Bicocca, Monza, Italy

Introduction: Neuroadrenergic overdrive occurs in obstructive sleep apnea syndrome (OSAS). However, the small sample size of the microneurographic studies, heterogeneity of the patients examines, presence of comorbidities, represented major weaknesses not allowing to precisely define the main features of the phoenomenon, particularly in non-obese patients.

Aim: The meta-analysis evaluated 850 patients recruited in 26 microneurographic studies, based on muscle sympathetic nerve activity (MSNA) quantification in uncomplicated OSAS of different clinical severity.

Methods: The evaluation was extended to the relationships of MSNA with heart rate (HR), anthropometric and blood pressure values, metabolic variables, apnea-hypopnea index (AHI) and oxygen saturation ( $\mathrm{sO} 2)$

Results: MSNA is activated markedly and almost homogeneously between studies, showing a progressive increase going from the healthy state to mild, moderate and severe OSAS (41.6, 48.3, $65.5 \mathrm{e}$ 70.7 bursts $/ 100$ heart beats, respectively, $\mathrm{P}<0.01$ ). Of special interest are the findings that (1) MSNA is significantly related to the AHI, a marker of OSAS severity $(\mathrm{r}=0.55, \mathrm{P}<0.02)$ and $\mathrm{sO} 2$ but not to body weight and body mass index, as it occurs in OSAS associated with obesity, and (2) HR is significantly and directly related to MSNA and AHI $(r=0.56$ and $r=0.46, P<0.03$ for both), thus representing a surrogate marker of the sympathetic overdrive.

Conclusions: OSAS, even when uncomplicated by other cardiometabolic disease, displays a marked sympathetic activation, reflexted by the MSNA and HR behaviour, becoming a target of therapeutic interventions aimed at exerting sympathomoderating effects, such as continuous positive airway pressure.

\section{PROGNOSTIC ROLE OF SERUM URIC ACID ON ALL-CAUSE AND CARDIOVASCULAR MORTALITY IN OLDER ADULTS: DATA FROM THE URIC ACID RIGHT FOR HEART HEALTH (URRAH) STUDY}

Giulia Rivasi ${ }^{1}$, Andrea Ungar ${ }^{1}$, Agostino Virdis ${ }^{2}$, Edoardo Casiglia ${ }^{3}$, Stefano Masi ${ }^{2}$, Giovambattista Desideri ${ }^{4}$, Claudio Ferri ${ }^{4}$, Maria Lorenza Muiesan ${ }^{5}$, Guido Grassi ${ }^{6}$, Claudio Borghi ${ }^{7}$ on behalf of the Working Group on Uric Acid and Cardiovascular Risk of the Italian Society of Hypertension (SIIA)

${ }^{1}$ Department of Geriatric and Intensive Care Medicine, Careggi Hospital and University of Florence, Florence, Italy, ${ }^{2}$ Department of Clinical and Experimental Medicine, University of Pisa, Pisa, Italy, ${ }^{3}$ Studium Patavinum, Department of Medicine, University of Padua, Padua, Italy, ${ }^{4}$ Department of Life, Health and Environmental Sciences, University of L'Aquila, L'Aquila, Italy, ${ }^{5}$ Department of Clinical and Experimental Sciences, University of Brescia, Brescia, Italy, ${ }^{6}$ Clinica Medica, Department of Medicine and Surgery, University of Milano-Bicocca, Monza, Italy, ${ }^{7}$ Department of Medical and Surgical Science, Alma Mater Studiorum University of Bologna, Bologna, Italy

Introduction: In older people, the role of serum uric acid (SUA) as risk factor for mortality is debated.

Aim: To investigate the association of SUA with all-cause and cardiovascular (CV) mortality in older adults participating in the large multicentre observational URic acid Right for heArt Health (URRAH) study and to explore the influence of age on SUA levels. Methods: 8000 URRAH participants aged $65+$ were included in the analysis. The predictive role of SUA was assessed using Cox regression models stratified according to the cut-off age of 75. SUA was tested as continuous and categorical variable (age-specific quartiles). The prognostic threshold of SUA for mortality was analysed using receiver operating characteristic curves. 
Results: SUA was independently associated with age, with a different trend in women and men. Among participants aged 65-74, multivariate Cox regression adjusted for $\mathrm{CV}$ risk factors and comorbidities identified an independent association of SUA with both all-cause (HR 1.169, 95\% CI 1.107-1.235) and CV mortality (HR 1.146, 95\% CI 1.064-1.235). The cut-off value of $4.8 \mathrm{mg} / \mathrm{dl}$ discriminated mortality status. In participants aged $75+$, we observed a J-shaped relationship of SUA with all-cause and CV mortality, with risk increasing at extreme SUA levels.

Conclusions: these results confirmed the predictive role of SUA for all-cause and CV mortality in older people, while revealing considerable age-related differences. Mortality risk increased at higher SUA levels in participants aged 65-74, with a prognostic threshold of 4.8 $\mathrm{mg} / \mathrm{dl}$. The relationship between SUA and mortality was J-shaped in oldest participants.

\section{SATURATED FATTY ACIDS IN ERYTHROCYTE MEMBRANES IN ADOLESCENTS WITH TYPE 1 DIABETES MELLITUS}

Denise Marcon ${ }^{1}$, Angela Tagetti ${ }^{1}$, Alice Giontella ${ }^{1}$, Giada Monamì ${ }^{1}$, Lorella Branz ${ }^{1}$, Stefano Bortolotti ${ }^{1}$, Claudia Piona ${ }^{2}$, Anita Morandi ${ }^{2}$, Laura Trento ${ }^{1}$, Pietro Minuz ${ }^{1}$, Claudio Maffeis ${ }^{2}$, Cristiano Fava ${ }^{1}$

\section{${ }^{1}$ Department of Medicine, University of Verona, Italy, ${ }^{2}$ Department of Surgery, Dentistry, Paediatrics and Gynaecology, University of Verona, Italy}

Introduction: Type 1 diabetes mellitus (T1DM) is associated with early development of atherosclerosis.

Aim: The aim of this study was to assess the association between saturated fatty acids (SFAs) contained in erythrocyte membranes and some indices of vascular damage in a sample of adolescents with T1DM.

Methods: Participants in regular follow-up at the Paediatric Diabetology Unit of Verona were sent for evaluation at the Vascular Laboratory of the General Medicine \& Hypertension Unit Central blood pressure (BP), Pulse Wave Velocity (PWV) and Pulse Wave Analysis (PWA) were measured by the SphygmoCor Xcel, carotid Intima Media Thickness (cIMT) and carotid distensibility (DC) by ultrasound and elaborated with a dedicated hardware. Blood samples were evaluated for erythrocyte membrane SFA, expressed as a percentage of total Fatty Acids (FAs).

Results: Hundred and forty-seven adolescents (78 males and 69 females, mean age 17, 25 $\pm 1,93$ years) were studied. Fifteen patients were classified as hypertensive $(10,2 \%)$ according to current European Guidelines. Thirteen patients had central systolic BP (cSBP) (8, $8 \%), 60$ cIMT $(40,8 \%), 49(33,3 \%)$ DC and 1 PWV $(0,7 \%)$ above the $95^{\text {th }}$ percentile for sex and age/height. We observed a correlation between total SFAs and cSB and z-cSBP ( $r=0,260$ and 0,269 respectively, $\mathrm{p}<0,05)$, not confirmed when each SFA was analysed separately. Furthermore, a positive correlation between total SFAs and either PWV or z-PWV was detectable $(r=0,241$ and 0,251 respectively, $\mathrm{p}<0,05)$. The latter association remained significant even after adjustment for age, sex, Body Mass Index (BMI), duration of diabetes, HbAlc, total cholesterol, triglycerides-HDL ratio. Total SFAs were also associated with BMI $(r=0,191, \mathrm{p}<0,05)$.

Conclusions: Erythrocyte membrane FAs are associated with vascular (cSBP and PWV) and anthropometric parameters in patients with T1DM. The amount of SFAs in membranes does not depend only on diet, as they are also neo-synthetized from glucidic precursors. Thus, it can be speculated that a bad glycemic control could translate into a higher substrate amount to produce the different SFAs. Further studies are needed to better understand the mechanisms and the regulations of these pathways.

\section{EFFECT OF BARIATRIC SURGERY ON BLOOD PRESSURE AND WORKLOAD-INDEXED PRESSURE DURING SUBMAXIMAL AND MAXIMAL EXERCISE}

Francesca Battista ${ }^{1}$; Marco Baldan ${ }^{1}$; Giulia Quinto ${ }^{1}$; Giulia Foccardi ${ }^{1}$; Marco Vecchiato ${ }^{1}$; Anna Centanini ${ }^{1}$; Silvia Bettini ${ }^{2}$; Andrea Gasperetti $^{1}$; Luca Busetto ${ }^{2}$; Daniel Neunhaeuserer ${ }^{1}$; Andrea Ermolao ${ }^{1}$

${ }^{I}$ Department of Medicine, Sports and Exercise Medicine Division, University of Padua, Padua, Italy; ${ }^{2}$ Department of Medicine, Internal Medicine 3, University Hospital of Padua, Padua, Italy

Introduction: Obesity is accompanied by increased resting blood pressure. Exaggerated blood pressure during exercise correlates with cardiovascular events independently from the baseline blood pressure levels. High Workload-indexed blood pressure (W-SBP) is significantly associated with mortality.

Aim: To evaluate the blood pressure response and W-SBP during a cardio-pulmonary exercise test (CPET) before and after bariatric surgery (BS).

Methods: 257 patients with severe obesity performed maximal incremental CPET one month before and six months after BS under the same protocol. The systolic blood pressure was measured at rest (SBPrest), at the same submaximal intensity of 3 METs (SBPsub$\max )$, at the exercise peak (SBPmax) and in the recovery phase. The submaximal and maximal W-SBP was calculated with a dedicated formula.

Results: Age was on average $45 \pm 10.3$ years, BMI before BS was $43.9 \pm 6.4 \mathrm{Kg} / \mathrm{m}^{2}$ and $73.5 \%$ were females. SBPrest decrease significantly after BS $(\Delta$ SBPrest $-10.2 \pm 15.8 \mathrm{mmHg} ; \mathrm{p}<0.001)$, also when considering percentage variation of pre BS values. SBPsubmax and SBPmax showed significant reduction after BS both as absolute change $(-15.0 \pm 19.7 \mathrm{mmHg}$ and $-10.3 \pm 25.1 \mathrm{mmHg} ; \mathrm{p}<0.001$, respectively) and as percentage change $(-9.6 \pm 13.0 \%$ and $-5.0 \pm$ $14.0 \% ; \mathrm{p}<0.001$, respectively). Furthermore, W-SBP decreased significantly during submaximal exercise $(-3.0 \pm 12.2 \mathrm{mmHg} / \mathrm{METs}$; $\mathrm{p} 0.001)$ and at peak of exercise $(-2.1 \pm 4.8 \mathrm{mmHg} / \mathrm{METs} ; \mathrm{p}<0.001)$, despite a higher exercise tolerance after BS (Figure 1). Lastly, also systolic blood pressure during the recovery phase showed a significant reduction $(-7.5 \pm 7.0 \mathrm{mmHg} ; \mathrm{p}<0.001)$. A Spearman's correlation analysis showed a significant but weak correlation between $\Delta \mathrm{SBP} \%$ submax and $\%$ of weight loss (rho $=0.138 ; \mathrm{p}=0.027$ ).

Conclusions: After BS, a marked reduction of resting and exercise blood pressure values was observed. W-SBPsubmax and W-SBPmax, as expression of load independent pressure response, decreased significantly, despite a significant increase of exercise tolerance after BS. The reduction of the submaximal blood pressure was weakly correlated with changes in body weight.

Figure

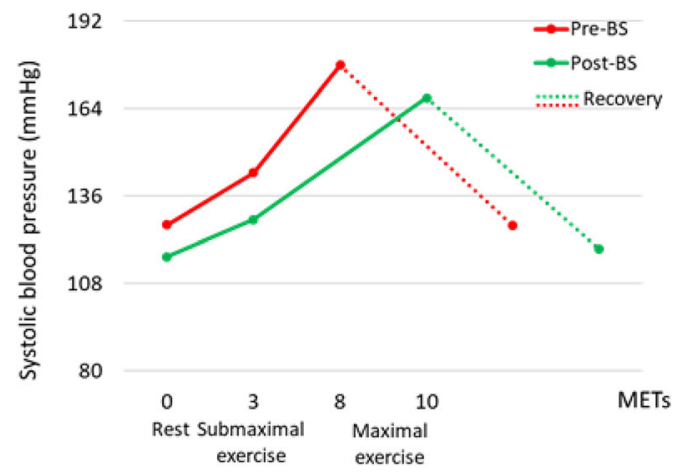




\section{EPICARDIAL \\ ADIPOSE \\ CARDIOVASCULAR HEMODYNAMICS \\ AND METABOLIC PROFILE IN HEART FAILURE WITH PRESERVED AND REDUCED EJECTION FRACTION}

Nicola Riccardo Pugliese ${ }^{1}$, Nicolò De Biase ${ }^{1}$, Matteo Mazzola ${ }^{2}$, Alessio Balletti ${ }^{1}$, Lavinia Del Punta ${ }^{1}$, Francesco Filidei ${ }^{1}$, Alessandro Mengozzi ${ }^{1}$, Alessandra Pieroni ${ }^{1}$, Agostino Virdis ${ }^{1}$, Stefano Taddei ${ }^{1}$, Stefano Masi ${ }^{1}$.

${ }^{1}$ Department of Clinical and Experimental Medicine, University of Pisa, Pisa, Italy, ${ }^{2}$ Cardiac, Thoracic and Vascular Department, University of Pisa, Pisa, Italy

Introduction: Epicardial adipose tissue (EAT) acts as a paracrine organ and could exert direct mechanical compression on the myocardium.

Aim: We investigated the impact of echocardiographic EAT thickness on metabolic profile and cardiovascular hemodynamics at rest and during exercise in heart failure (HF) patients with reduced (HFrEF) and preserved (HFpEF) ejection fraction

Methods: We prospectively enrolled 393 consecutive HF outpatients (205 HFrEF, $188 \mathrm{HFpEF}$ ) referred to the our hospital due to dyspnoea and/or effort intolerance. We performed a resting clinical and biohumoral evaluation, followed by combined cardiopulmonaryechocardiography exercise stress. The protocol also included 44 healthy controls.

Results: Patients with HFpEF displayed the greatest EAT thickness (median $8 \mathrm{~mm}$, interquartile range [IQR] 4-12 mm), while those with HFrEF had thinner EAT (median $3 \mathrm{~mm}$, IQR 2-6 mm) than controls (median $5 \mathrm{~mm}$, IQR 3-7 mm; p < 0.0001). In HFrEF, EAT thickness was inversely associated with natriuretic peptides, Troponin $\mathrm{T}$, and C-reactive protein levels. In $\mathrm{HFpEF}$, it was directly correlated with Troponin- $\mathrm{T}$ and $\mathrm{C}$-reactive protein levels. EAT thickness was inversely correlated with peak oxygen consumption $\left(\mathrm{VO}_{2}\right)$ and peripheral oxygen extraction $\left(\mathrm{AVO}_{2}\right.$ diff $)$ in $\mathrm{HFpEF}$, while a direct association was observed in HFrEF. EAT resulted in an independent predictor of peak $\mathrm{VO}_{2}$ and $\mathrm{AVO}_{2}$ diff in $\mathrm{HFrEF}$ and $\mathrm{HFpEF}$ regardless of body mass index, but the relationships were direct in HFrEF (standard regression coefficient [SRC] for peak $\mathrm{VO}_{2}: 0.18, \mathrm{p}=0.02$; SRC for peak $\mathrm{AVO}_{2}$ diff: $0.17, \mathrm{p}=0.03$ ) and indirect in $\mathrm{HFpEF}$ (SRC for peak $\mathrm{VO}_{2}$ : -0.33, $\mathrm{p}<0.0001$; SRC for peak $\mathrm{AVO}_{2}$ diff: $-0.25, \mathrm{p}<0.0001$ ). The analysis of cardiac mechanics revealed that thinner EAT was associated with worse left ventricle systolic dysfunction (average $S$ ') and remodeling (3D left ventricle mass) in HFrEF. In HFpEF, increased EAT was related to more severe left atrio-ventricular (left atrium reservoir strain/left ventricle global longitudinal strain) and right ventriculo-arterial (tricuspid annular plane systolic excursion/ systolic pulmonary artery pressure) coupling.

Conclusions: In HFpEF, EAT accumulation is associated with worse hemodynamic and metabolic profile. In HFrEF, conversely, EAT thinning portends more severe LV dysfunction and impaired global functional capacity.

\section{PROGNOSTIC SIGNIFICANCE OF EPICARDIAL ADIPOSE TISSUE IN HEART FAILURE WITH PRESERVED AND REDUCED EJECTION FRACTION}

Nicola Riccardo Pugliese ${ }^{1}$, Nicolò De Biase ${ }^{1}$, Matteo Mazzola ${ }^{2}$, Alessio Balletti ${ }^{1}$, Lavinia Del Punta ${ }^{1}$, Francesco Filidei ${ }^{1}$, Alessandro Mengozzi ${ }^{1}$, Alessandra Pieroni ${ }^{1}$, Agostino Virdis ${ }^{1}$, Stefano Taddei ${ }^{1}$, Stefano Masi ${ }^{1}$

${ }^{1}$ Department of Clinical and Experimental Medicine, University of Pisa, Pisa, Italy, ${ }^{2}$ Cardiac, Thoracic and Vascular Department, University of Pisa, Pisa, Italy

Introduction: Increased epicardial adipose tissue (EAT) thickness correlates with metabolic syndrome, insulin resistance, microvascular dysfunction and enhanced pericardial restraint.

Aim: We measured echocardiographic EAT thickness in heart failure (HF) patients with reduced (HFrEF) or preserved (HFpEF) ejection fraction to determine whether EAT could bear prognostic significance at clinical follow-up.

Methods: We prospectively enrolled 393 consecutive HF outpatients 393 subjects (205 HFrEF, $188 \mathrm{HFpEF}$ ) referred to our hospital due to dyspnoea and/or effort intolerance. We performed a resting clinical and biohumoral evaluation, followed by combined cardiopulmonaryechocardiography exercise stress. A composite of cardiovascular death and HF-related hospitalization was chosen as endpoint during follow-up.

Results: Patients with HFpEF displayed higher EAT thickness (median $8 \mathrm{~mm}$, interquartile range [IQR] 4-12 mm) than HFrEF (median $3 \mathrm{~mm}$, IQR 2-6 mm; p < 0.0001). During a median follow-up of 20.9 months (IQR 15-25 months), we reported 34 cardiovascular deaths and $146 \mathrm{HF}$ hospitalizations, with no significant differences between the two HF subsets. EAT predicted adverse events independently from body mass index and well-established markers of poor prognosis (e.g., NT-proBNP, peak oxygen consumption). The risk of adverse events increased with increasing EAT thickness in HFpEF and EAT thinning in HFrEF. Kaplan-Meier analyses for the composite endpoint showed that in $\mathrm{HFpEF}$, the survival probability was significantly lower in patients with thicker EAT than those with thinner EAT. In HFrEF, conversely, patients with thicker EAT had a higher survival probability than those with reduced EAT thickness.

Conclusions: EAT accumulation is more marked in HFpEF than HFrEF and carries different prognostic meanings in the two subsets. In HFpEF, EAT thickening portends adverse outcome, which may be due to increased mechanical restraint and secretion of pro-inflammatory and pro-atherogenic adipokines. In HFrEF, greater EAT thickness seems to have a protective role, while EAT thinning is associated with a worse prognosis, probably reflecting a catabolic state (e.g. cardiac cachexia). Larger studies will clarify whether EAT is a bystander or an active player in the different HF phenotypes. 


\section{THE FUNCTIONAL VASCULAR DAMAGE IS ASSOCIATED TO LDL CHOLESTEROL IN HYPERTENSIVE PATIENTS WITH NORMAL TOTAL CHOLESTEROLEMIA}

Pietro Nazzaro, Adele Nardecchia, Maria Contini, Gianfranco Amodio, Gabriella Schirosi, Lorenzo Debenedittis, Gabriella Aceto, Serena Calò, Mariangela Lisena

Dept. Biomedical Sciences and Human Oncology, Hypertension Unit "AM. Pirrelli", University of Bari "Aldo Moro", Bari, Italy

Introduction: Different studies highlighted the association between elevated LDL cholesterol (LDL-C) and cerebrocardiovascular diseases.

Aim: To discern the association of LDL-C with vascular impairment in hypertensives with total cholesterol (TOT-C) normal levels, established by diet or therapy (simvastatin 20-40 mg/atorvastatin 10-20 mg), within recommended limit $(<190 \mathrm{mg} / \mathrm{dl})$.

Methods: We studied 264 hypertensives, treated by ACEi or ARB, with similar history and hypertensive state, validated by ABPM, partitioned in tertiles by LDL-C (mg/dl): 72 Low $(38,4-72,2) ; 135$ Med (73, 2-103, 4); 57 High (103, 8-131, 2). They, after medical visit (off) and detection of the metabolic syndrome factors (nMS), underwent tonometry to obtain central (car) SBP, arterial stiffness index (PWVcf) and 18-item questionnaire to evaluate the cognitive impairment (COGtot).

Results: Patients presented similar SBP/DBP off $(133 \pm 11 / 81 \pm 7$ vs $135 \pm 17 / 81 \pm 10$ vs $136 \pm 12 / 79 \pm 9 \mathrm{mmHg}$ ), smoke habit, BMI $(29,9 \pm 5,7$ vs $30,6 \pm 6,2$ vs $29,4 \pm 3,9)$ and body composition by bioimpedance.

\section{METABOLIC SYNDROME IS RELATED TO VASCULAR STRUCTURAL ALTERATIONS BUT NOT TO FUNCTIONAL ONE BOTH IN HYPERTENSIVES AND HEALTHY SUBJECTS}

Filippo Leidi ${ }^{1,2}$, Alessandro Maloberti ${ }^{1,2}$, Michele Bombelli ${ }^{2}$, Massimiliano Monticelli ${ }^{1,2}$, Giacomo Ruzzenenti ${ }^{1,2}$, Marco Biolcati $^{1,2}$, Valentina Giani ${ }^{1,2}$ Benedetta De Chiara ${ }^{1}$, Salvatore Riccobono $^{1}$, Guido Grassi ${ }^{2}$ and Cristina Giannattasio ${ }^{1,2}$

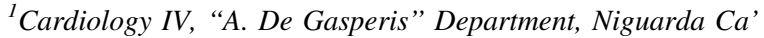
Granda Hospital, Milan, Italy, ${ }^{2}$ School of Medicine and Surgery, Milano-Bicocca University, Milan, Italy

Introduction: Metabolic Syndrome (MS) has been related to an impairment in arterial structural and functional properties with heterogeneous results.

Aim: In this study we focused on the effects of MS on arterial carotid-femoral Pulse Wave Velocity (PWV) and common carotid Intima Media Thickness (IMT) in two different populations, one of hypertensive patients and one of healthy controls.

Methods: We enrolled 816 consecutive hypertensive (HT) and 536 healthy controls. Vascular structural (IMT) and functional (PWV) properties were evaluated. NCEP-ATP-III criteria were used for diagnosis of MS.

Results: MS was diagnosed in $26.9 \%$ and $6.9 \%$ in hypertensive and control subjects, respectively. PWV was similar in controls with and without MS $(7.7 \pm 1.9$ vs $7.6 \pm 1.1 \mathrm{~m} / \mathrm{s}, \mathrm{p}=0.69)$, while IMT was higher in controls with than those without MS $(0.64 \pm 0.18$ vs $0.57 \pm$

\begin{tabular}{|l|c|c|c|c|c|c|}
\hline pts/var & TOT-C & LDL-C & nMS & SBPcar & PWVcf & COGtot \\
\hline Low LDL-C & $137 \pm 12$ & $65 \pm 6$ & $2.8 \pm 1.4$ & $121 \pm 15$ & $8.9 \pm 2.2$ & $22.1 \pm 6.6$ \\
\hline Med LDL-C & $165 \pm 12 * * *$ & $98 \pm 9 * * *$ & $2.9 \pm 1.3$ & $127 \pm 15^{*}$ & $11.5 \pm 3.3 * * *$ & $25.5 \pm 7.2 * *$ \\
\hline High LDL-C & $176 \pm 6 * * * \wedge \wedge \wedge$ & $114 \pm 8 * * * \wedge \wedge \wedge$ & $2.6 \pm 1.1$ & $131 \pm 12 * * *$ & $12.2 \pm 3.3 * * *$ & $31.2 \pm 9.6 * * * \wedge \wedge \wedge$ \\
\hline
\end{tabular}

(mean \pm s.d; * $p<.05, * * p<.01, * * * p<.001$ vs Low LDL-C; ${ }^{\wedge} p<.05, \wedge \wedge p<.01, \wedge \wedge \wedge p<.001$ vs Med LDL-C).

Pearson test, controlled by age, SBP/DBPoff, C-TOT e C-HDL, showed that LDL-C was associated with SBPcar (.199*), PWVcf $\left(.217^{* *}\right)$ and COGtot $\left(.285^{* * *}\right)$.

Conclusions: The findings indicate that LDL-C, since nearby at the $70 \mathrm{mg} / \mathrm{dl}$ or more may represent a prognostic marker of vascular damage and reduced arterial compliance and mild cognitive impairment even in hypertensive patients with recommended total cholesterolemia.
$0.13 \mathrm{~mm}, \mathrm{p}=0.02)$. Hypertensives with MS were older $(57.9 \pm 12.2$ vs $52.7 \pm 14.1$ years, $\mathrm{p}<0.001)$ and showed higher PWV $(9.0 \pm 2.3$ vs $8.4 \pm 2.1 \mathrm{~m} / \mathrm{s}, \mathrm{p}=0.001)$ and IMT $(0.72 \pm 0.22$ vs $0.65 \pm 0.17$ $\mathrm{mm}, \mathrm{p}<0.001)$ than those without MS, however at the age-adjusted analysis only the difference in IMT was confirmed $(\mathrm{p}=0.007)$. Regression models showed that MS was an independent determinant of IMT in both controls $(\beta=0.08, p=0.03)$ and hypertensives $(\beta=$ $0.08, \mathrm{p}=0.01)$, but not of PWV either in controls $(\beta=0.006, \mathrm{p}=$ 0.886 and $\beta=0.04, p=0.19$, respectively).

Conclusions: the main finding of our work is that MS is a significant determinant of IMT while this is not the case for PWV. This result has been confirmed both in hypertensive subjects and in healthy controls. 


\section{HYPERURICEMIA PREVALENCE IN HEALTHY SUBJECTS AND ITS RELATIONSHIP WITH CARDIOVASCULAR TARGET ORGAN DAMAGE}

Valentina Giani ${ }^{2}$, Alessandro Maloberti ${ }^{1,2}$, Marco Biolcati ${ }^{2}$ Lucia Occhi $^{1,2}$, Sun Jinwei ${ }^{2}$, Enzo Grasso ${ }^{2}$, Chiara Tognola ${ }^{2}$, Martina Milani $^{2}$, Michele Bombelli ${ }^{2,3}$, Guido Grassi ${ }^{2,3}$, Cristina Giannattasio $^{1,2}$

${ }^{1}$ Cardiology IV, “A.De Gasperis” Department, ASTT GOM Niguarda Ca' Granda, Milan, Italy, ${ }^{2}$ University of Milano-Bicocca, Milan, Italy, ${ }^{3}$ Laboratory Medicine, San Gerardo Hospital, ASST Monza, Italy

Introduction: Heterogeneous results have been obtained in the relationship between Uric Acid (UA) and Target Organ Damage (TOD).

Aim: In the present study we sought to assess the prevalence of hyperuricemia in healthy subjects as well as the role of UA in determining TOD. We evaluated vascular, cardiac and renal TODs in the whole population as well as sub-grouped by gender.

Methods: 379 blood donors participated at the present analysis. TOD was evaluated as Pulse Wave Velocity (PWV), Left Ventricular Mass Index (LVMI) carotid Intima-Media Thickness (IMT) and Glomerular Filtration Rate (GFR). Hyperuricemia was defined with the classic cut-off ( $>7.0$ in men and $>6.0 \mathrm{mg} / \mathrm{dL}$ in women) but also with a most recently defined one $(5.6 \mathrm{mg} / \mathrm{dL}$ for both sex).

Results: Hyperuricemia was present in $6.3 \%$ of the whole population (7.3\% males, $2.8 \%$ females) considering the classic cut-off, while, with the recently identified one, it was present in $28.2 \%$ of the whole population (37.3\% males, $4.7 \%$ females). Despite all the evaluated TODs significantly correlated with UA, linear multivariate regression analysis showed that none of them, except for GFR, displayed UA as a significant covariate. Similar figures were found also when both correlation and linear regression analyses were repeated in the two genders separately.

Conclusions: Hyperuricemia is an important problem also in healthy subjects and its prevalence could further increase if lower cut-off will be used. In this specific population, UA is statistically associated only with renal impairment while this was not the case for cardiac and vascular damage.

\section{Atherosclerosis and Inflammation}

\section{THE SELECTIVE ACTIVATION OF FORMYL PEPTIDE RECEPTOR 2 PREVENTS THE INFLAMMATORY AND PRO-CALCIFIC DIFFERENTIATION OF INTERSTITIAL AORTIC VALVE CELLS}

Maristella Donato ${ }^{1}$, Chiara Bolego ${ }^{1}$, Elisabetta Faggin ${ }^{2}$, Alessandro Bressan $^{2}$, Francesco Cinetto ${ }^{2,3}$, Chiara Felice ${ }^{2,3}$, Carlo Agostini ${ }^{2,3}$, Marcello Rattazzi $i^{2,3}$

${ }^{1}$ Department of Pharmaceutical and Pharmacological Sciences, University of Padua, Padua, Italy, ${ }^{2}$ Department of Medicine-DIMED, University of Padua, Padua, Italy, ${ }^{3}$ Medicina Generale $I^{\wedge}, \mathrm{Ca}^{\prime}$ Foncello Hospital, Treviso, Italy

Introduction: Calcific aortic valve disease (CAVD) is the most common valvulopathy in the general population; however, no effective pharmacological therapy has proven to halt or delay its progression.

Aim: We aimed to investigate the effects of the activation of Formyl Peptide Receptor 2 (FPR2), a known receptor for lipoxins, resolvins and anti-inflammatory molecules, on the pro-calcific and inflammatory differentiation of interstitial aortic valve cells (VICs).

Methods: A clone of primary VICs seeded on culture plates or type I collagen scaffolds was treated with lipopolysaccharide (LPS, $500 \mathrm{ng}$ / $\mathrm{mL}$ ) for 12 days to acquire a pro-calcific profile, with or without the concomitant supplementation of two synthetic FPR2 agonists (MMK1, $50 \mu \mathrm{M}$ and Ac2-26, 3.2 $\mu \mathrm{M}$ ). At the end of the treatment, proteins and RNA were extracted for western blotting and gene expression analysis (RT-PCR). Alkaline phosphatase (ALP) activity and calcium deposition in collagen scaffolds were determined through colorimetric assays. The expression of FPR2 on human pathological and healthy aortic valves was investigated through immunohistochemistry and gene expression analysis.

Results: The gene expression analysis on human valves showed an increase in ALP, IL-6 and FPR2 levels in pathological valves compared to healthy tissue. Concurrently, the in vitro exposure of VICs to LPS increased the expression of inflammatory cytokines (such as IL-1beta, IL-6, TNF-alpha), ALP and FPR2. Moreover, it induced the deposition of calcium on collagen scaffolds. The treatment of cultured VICs with two selective FPR2 agonists (MMK1 and Ac2-26) reduced the overexpression of ALP $(\mathrm{p}<0.05)$ and inflammatory cytokines (such as IL6, $\mathrm{p}<$ 0.05 ) induced by LPS. The same treatments were effective in reducing the deposition of calcium in collagen scaffolds $(\mathrm{p}<0.05)$.

Conclusions: The receptor FPR2 is overexpressed under pathological conditions and its selective activation is associated with reduced procalcific and inflammatory differentiation of VICs. These promising data offer new insights for a novel therapeutic strategy for CAVD.

\section{SUPERVISED AND UNSUPERVISED LEARNING TO DEFINE THE CARDIOVASCULAR RISK OF PATIENTS ACCORDING TO AN EXTRACELLULAR VESICLE MOLECULAR SIGNATURE}

Jacopo Burrello ${ }^{1,2}$, Alessio Burrello ${ }^{3}$, Elena Vacchi ${ }^{4,}$, , Giovanni Bianco $^{6}$, Elena Caporali ${ }^{1}$, Lorenzo Grazioli ${ }^{1}$, Martina Amongero ${ }^{7}$, Sara Bolis $^{1}$, Giuseppe Vassalli ${ }^{5,8}$, Carlo Cereda ${ }^{6}$, Paolo Mulatero ${ }^{2}$, Benedetta Bussolati ${ }^{9}$, Giorgia Melli ${ }^{4}, 5$, Giovanni G Camici $^{10}$, Silvia Monticone $^{2}$, Lucio Barile ${ }^{1,5}$

${ }^{1}$ Laboratory for Cardiovascular Theranostics, Cardiocentro Ticino Institute, Lugano, Switzerland, ${ }^{2}$ Division of Internal Medicine and Hypertension Unit, Department of Medical Sciences, University of Turin, Turin, Italy, ${ }^{3}$ Department of Electrical, Electronic and Information Engineering (DEI), University of Bologna, Bologna Italy, ${ }^{4}$ Laboratory for Biomedical Neurosciences, Neurocenter of Southern Switzerland, Ente Ospedaliero Cantonale, Lugano, Switzerland, ${ }^{5}$ Faculty of Biomedical Sciences, Università della Svizzera Italiana, Lugano, Switzerland, ${ }^{6}$ Neurology Clinic, Stroke Center, Neurocenter of Southern Switzerland, Lugano, Switzerland, ${ }^{7}$ Department of Mathematical Sciences G. L. Lagrange, Polytechnic University of Turin, Turin, Italy, ${ }^{8}$ Cellular and Molecular Cardiology Laboratory, Cardiocentro Ticino Institute, Lugano, Switzerland, ${ }^{9}$ Department of Molecular Biotechnology and Health Sciences, University of Turin, Italy, ${ }^{10}$ Center for Molecular Cardiology, University of Zürich, Switzerland

Introduction: Secreted extracellular vesicles (EVs) are membranebound nanoparticles released from cells. Since their content reflect the 
specific signatures of cellular activation and injury, EVs display a strong potential as biomarkers in the cardiovascular (CV) field.

Aim: We aimed at dissecting a specific EV signature able to stratify patients according to their $\mathrm{CV}$ risk and likelihood to develop fatal $\mathrm{CV}$ events.

Methods: A total of 404 patients were included in the analysis. For each subject, we evaluated several CV risk indicators (age, sex, BMI, hypertension, hyperlipidemia, diabetes, coronary artery disease, chronic heart failure, chronic kidney disease, smoking habit, organ damage) and the likelihood of fatal CV events at 10 years, according to the SCORE charts of the European Society of Cardiology. Serum EVs were isolated by immuno-capture and analyzed for the expression of $37 \mathrm{EV}$ surface antigens by flow cytometry. Unsupervised and supervised learning algorithms were applied for clustering patients according to $\mathrm{CV}$ risk.

Results: Based on expression levels of EV antigens, unsupervised learning classified patients into three clusters (cluster I, 288 patients; cluster II, 86 patients; cluster III, 30 patients). Prevalence of hypertension, diabetes, chronic heart failure and organ damage (defined as left ventricular hypertrophy and/or microalbuminuria) progressively increases from cluster I to cluster III, with an average 6.9-fold increase. Several EV antigens, including markers from platelets (CD41b-CD42a-CD62P), leukocytes (CD1c-CD2-CD3-CD4-CD8CD14-CD19-CD20-CD25-CD40-CD45-CD69-CD86), and endothelium (CD31-CD105) were independently associated to the $\mathrm{CV}$ risk indicators and correlated to age, blood pressure, glucometabolic profile, renal function, and SCORE risk. EV specific signature obtained by supervised learning allowed the accurate classification of patients according to their 10-year risk of future CV events, as estimated with the SCORE risk charts.

Conclusions: EV profiling, obtainable from minimally-invasive blood sampling, may be integrated into $\mathrm{CV}$ risk stratification, displaying a potential role in the tailored management of these patients.

\section{NETOSIS IN ATHEROTHROMBOTIC DISORDERS}

Bressan Alessandro ${ }^{1}$, Faggin Elisabetta ${ }^{1}$, Tonon Luca ${ }^{1,2}$, Donato Maristella $^{3}$, Tiepolo Marta ${ }^{1,2}$, Nardin Chiara ${ }^{1,2}$, Cinetto Francesco ${ }^{1,2}$, Felice Carla ${ }^{1,2}$, Buso Roberta ${ }^{1,2}$, Agostini Carlo ${ }^{1,2}$, Rattazzi Marcello $^{1,2}$

${ }^{1}$ Department of Medicine-DIMED, University of Padua, Padua, Italy, ${ }^{2}$ General Medicine I^, Ca' Foncello Hospital, Treviso, Italy, ${ }^{3}$ Department of Pharmaceutical and Pharmacological Science, University of Padua, Padua, Italy

Introduction: Neutrophils have been involved in the pathogenesis and progression of cardiovascular diseases. In particular, it has been suggested that NETosis, a specific cell death mechanism of neutrophils, may play an active role in the onset of acute thrombotic diseases.

Aim: to study the expression of neutrophil activation and NETosis biomarkers in subjects with acute venous thromboembolic disease (VTE), acute coronary syndrome (ACS) and acute cerebrovascular disease (CVD).

Methods: 165 patients with acute thrombotic events (60 acute coronary syndromes, 55 venous thrombotic events, 50 cerebrovascular disease) and 70 subjects without clinical/instrumental evidence of acute cardiovascular disease were enrolled. Clinical and biochemical data were collected for each patient, and circulating levels of NETosis markers (nucleosomes, MPO-DNA complex) and neutrophils activation markers (MPO, NGAL, elastase and lactoferrin) were measured.
Results: The analysis of NETosis biomarkers showed a significant increase in circulating MPO-DNA levels in patients with ACS, VTE and CVD compared to control subjects $(\mathrm{p}<0.001)$. No significant differences in MPO-DNA levels were observed within clinical subgroups of different pathological conditions (STEMI vs NSTEMI, atrial fibrillation vs non-atrial fibrillation, venous thromboembolism provoked vs unprovoked). A logistic regression analysis showed that plasma levels of MPO-DNA are associated with the presence of ACS regardless of the presence of traditional risk factors $(p=0.002)$. Concerning neutrophil activation biomarkers only NGAL showed a significant increase in circulating levels in all thrombotic conditions compared to the control group ( $\mathrm{p}<0.05$ ).

Conclusions: This study demonstrates that NETosis represents a pathological process common to major acute thrombotic diseases.

\section{EFFICACY AND SAFETY OF INCLISIRAN: A SYSTEMATIC REVIEW AND POOLED ANALYSIS OF AVAILABLE CLINICAL STUDIES}

Arrigo F. G. Cicero ${ }^{1,2}$, Federica Fogacci ${ }^{1,2}$, Alberto Zambon ${ }^{3,4}$, Peter P. Toth ${ }^{5}$, Claudio Borghi ${ }^{1,2}$

${ }^{1}$ Hypertension and Cardiovascular Risk Factors Research Group, Medical and Surgical Sciences Department, Sant'Orsola-Malpighi University Hospital, Bologna, Italy, ${ }^{2}$ IRCCS Azienda OspedalieroUniversitaria di Bologna, Bologna, Italy, ${ }^{3}$ IRCCS MultiMedica, Sesto S. Giovanni MI, Italy, ${ }^{4}$ Department of Medicine-DIMED, University of Padua, Padua, Italy, ${ }^{5}$ CGH Medical Center, Sterling, Illinois, and Cicarrone Center for the Prevention of Cardiovascular Disease, Johns Hopkins University School of Medicine, Baltimore, MD, USA

Introduction: Inclisiran, a small interfering RNA, is a new drug that inhibits proprotein convertase subtilisin/kexin type 9 (PCSK9) synthesis.

Aim: To assess the efficacy and safety of inclisiran treatment with a systematic review of the literature and a meta-analysis of the available clinical studies.

Methods: A systematic literature search was conducted in order to identify clinical trials assessing the effect on lipoproteins and the safety profile of inclisiran.

Results: Data were pooled from 5 clinical studies, which included 4226 subjects. Meta-analyses of data suggested that the multiple-dose regimen of inclisiran yielded a significant reduction in serum levels of proprotein convertase subtilisin/kexin type $9(\mathrm{MD}=-78.23 \%, 95 \% \mathrm{CI}$ : $-86.74,-69.71)$ and low-density lipoprotein cholesterol $(\mathrm{MD}=$ $45.48 \%$, 95\% CI: $-50.36 \%,-40.61 \%$ ) throughout the studies. Furthermore, treatment with inclisiran significantly affected total cholesterol (MD $=-13.67 \%, 95 \% \mathrm{CI}:-20.78 \%,-6.57 \%)$, high-density cholesterol $(\mathrm{MD}=8.29 \%$, 95\%CI: $4.66 \%, 11.93 \%)$, non-HDL cholesterol (MD $=-39.45 \%$, 95\% CI: $-43.6 \%,-35.31 \%$ ), apolipoprotein $\mathrm{B}(\mathrm{MD}=-34.58 \%, 95 \% \mathrm{CI}:-38.78 \%,-30.78 \%)$ and lipoprotein (a) $(\mathrm{MD}=-20.9 \%, 95 \% \mathrm{CI}:-25.8 \%,-15.99 \%)$. Multiple-dose regimens of inclisiran were associated with increased risk of injection-site reactions (any reaction: $\mathrm{OR}=5.86,95 \% \mathrm{CI}$ : $3.44,9.98$; mild reactions: $\mathrm{OR}=5.19,95 \% \mathrm{CI}: 1.68,16.07$; moderate reactions: $\mathrm{OR}=13.37$, 95\%CI: $3.17,56.46)$, and bronchitis (OR $=1.58,95 \% \mathrm{CI}: 1.10,2.26$ ), while the incidence of the pre-specified exploratory $\mathrm{CV}$ endpoint significantly decreased at 18 months $(\mathrm{OR}=0.74,95 \% \mathrm{CI}$ : $0.58,0.94)$. Conclusions: Inclisiran has favourable effects on lipids serum levels and an acceptable safety profile. Further well-designed studies are needed to explore its longer-term safety. 


\section{AN ELEVATED NEUTROPHIL-LYMPHOCYTE RATIO INCREASES THE RISK OF CARDIOVASCULAR EVENTS AND OF RENAL FUNCTION DECLINE IN HYPERTENSIVE PATIENTS WITH CKD}

Giuseppe Mule', Valeria Tranchida, Alessandra Sorce, Nicola Sinatra, Ettore Mancia, Maria Giovanna Vario, Santina Cottone

ESH Hypertension Excellence Centre and Nephrology Unit of the University of Palermo, Palermo, Italy

Introduction: Many epidemiological studies have shown that lowgrade chronic inflammation, estimated through the neutrophil-lymphocyte ratio (NLR), is linked to traditional and non-traditional cardiovascular $(\mathrm{CV})$ risk factors.

Aim: The aim of our study was to investigate the potential prognostic role of NLR regarding the progression of chronic kidney disease (CKD) and the development of CV events in a cohort of renal and hypertensive patients.

Methods: In this retrospective observational study, 403 patients consecutively attending our Nephrology and Hypertension clinics were included. At the baseline observation and control visits in all patients, in addition to the clinical-anamnestic evaluation, and the measurement of blood pressure values and anthropometric indices, a complete blood count and biohumoral data regarding renal function, lipid profile and to serum electrolytes were obtained.

Results: Median follow-up time was 38.95 [27-75] months. Patients were divided into two groups based on an NLR cut-off of 1.96, which resulted from the analysis of ROC curves as the optimal NLR value for identifying patients at greatest risk of $\mathrm{CV}$ events. During the observation period $103 \mathrm{CV}$ events occurred, 72 of which were in the group with the highest NLR. Analyzing the ROC curves, an NLR value of 1.96 was found to have a sensitivity of $78.7 \%$ and a specificity of $51.7 \%$ (AUC $=$ $0.672, \mathrm{P}=<0.0001$ ) in predicting the $\mathrm{CV}$ outcome. Multivariate Cox regression analyses showed that an NLR $>1.96$ predicted CV events, even after correction for gender, BMI, diabetes, BP values, dyslipidemia and hyperuricaemia (HR: $2.54 ; p=0.0003$ ). A more rapid progression of renal disease (defined as an annual decline in GFR $>3 \mathrm{ml} / \mathrm{min} / \mathrm{m}^{2}$ ) was observed in 170 patients. The ability of an NLR $>1.96$ to predict the risk of CKD progression was found to be independent of age, sex, obesity, dyslipidemia, hyperuricaemia, diabetes and BP values (HR: 1.60, $\mathrm{p}=$ 0.0123 ). An NLR value of 1.96 was found to have a sensitivity of $74 \%$ and a specificity of $63 \%(\mathrm{AUC}=0.582 ; \mathrm{p}=0.0054)$ in predicting a more rapid decline in renal function.

Conclusions: In conclusion, the results of our study suggest that, in patients with $\mathrm{CKD}$, an elevated neutrophil/lymphocyte ratio represents an independent predictor not only of the development of cardiovascular events, but also of a more rapid progression of renal disease.

\section{THE SUBFORNICAL ORGAN}

\section{AND THE PARAVENTRICULAR NUCLEUS} OF THE HYPOTHALAMUS MODULATE SPLENIC IMMUNITY IN RESPONSE TO HYPERTENSIVE STIMULI SUCH AS ANGIOTENSIN II

\footnotetext{
${ }^{1}$ Marialuisa Perrotta, ${ }^{2}$ Fabio Pallante, ${ }^{2}$ Lorenzo Carnevale, ${ }^{2}$ Francesco Mastroiacovo, ${ }^{2}$ Giuseppe Cifelli, ${ }^{1,2}$ Giuseppe Lembo,

${ }^{1,2}$ Daniela Carnevale
}

${ }^{I}$ Departement of Molecular Medicine, Sapienza University of Rome, Rome, Italy, ${ }^{2}$ Department of AngioCardioNeurology and Translational Medicine, IRCCS Neuromed, Pozzilli, Italy
Introduction: Angiotensin II (AngII) acts through neuronal AT1 (AT1R) receptors in the main cardiovascular control centers of the brain. The subfornical organ (SFO) is located in a ventricular region at the level of which the blood brain barrier is permeable to circulating substances such as AngII. Furthermore, it is structurally and functionally connected to the paraventricular nucleus of the hypothalamus (PVN) and both are rich in AT1R. In our previous studies, we have shown that chronic administration of AngII in mice activated immune responses in the spleen by the activation of the peripheral nervous system.

Aim: Brain areas where AT1R receptors are activated following AngII administration remain to be clarified.

Methods: To study the role of SFO, we injected, by stereotaxis, a recombinant adenovirus, encoding a Cre-recombinase and a GFP (AdCreGFP), or only GFP as control in the SFO of AT1aflox (mutants with loxP sites upstream and downstream to the exon 3 of the AT gene type 1a) or AT1a KO-SFO mice. To study the role of PVN, we crossed AT1aflox mice with Sim1Cre mice or AT1a KO-PVN mice. Results: We chronically treated mice of the two genotypes with AngII for 28 days by osmotic minipumps, observing that both AT1a $\mathrm{KO}-\mathrm{SFO}$ mice and the AT1a KO-PVN mice showed protection from increased blood pressure levels, measured by tail cuff plethysmography, after chronic infusion of AngII, when compared to their respective controls, Then, we also observed a reduction of $\mathrm{T}$ lymphocytes egression and migration from the spleen towards target organs of hypertension such as the kidneys. In fact, by immunohistochemistry we observed that both AT1a KO-SFO and AT1a KOPVN mice showed reduced infiltration of CD8 and CD4 T cells in kidneys and a protection from structural tissue damage, generally observed in WT mice after chronic infusion with AngII. Finally, to investigate the brain areas in which systemic AngII was capable to activate the neuronal signaling by ATlaR, we injected fluorescent labelled AngII in the right femoral vein, observing by confocal microscopy analysis that it accumulates in the SFO and also in the PVN.

Conclusions: We found that the SFO-PVN axis is the main integration nucleus of the hypertensive signal coming from the peripheral system.

\section{Coronavirus and Cardiovascular Risk Factors}

\section{MONOCENTRIC COHORT RETROSPECTIVE OBSERVATIONAL STUDY ON COVID-19 PATIENTS AIMED AT EXPLORING THE FACTORS OF CARDIOVASCULAR DEATH}

Chiara Grasselli, Angela Muoio, Angelo Ghirarduzzi

Hypertension Center, Cardiovascular Medicine Unit-AUSL-IRCCS of Reggio Emilia, Italy

Introduction: Several factors have been proposed to explain the high mortality rate of the Coronavirus disease epidemic 2019 (COVID-19), including hypertension and treatment with renin-angiotensin-aldosterone (RAAS) system inhibitors. Age and multimorbidity could be confounding factors.

Aim: Our Hypertension Center enlisted 261 Covid 19 patients, admitted from March $5^{\text {th }}$ to April $30^{\text {th }}, 2020$ at our Cardiovascular Medicine Unit (males $61.7 \%, 71 \pm 0.5$ years), of which 191 patients (73.2\%) had comorbidity.

Methods: The $65.5 \%$ of patients had previous cardiovascular diseases, of which hypertension was the most represented (61.7\%). In Table 1 the demographic characteristics of our patients at the onset, 
with fever in first place, followed by dyspnea/tachypnea and cough and in Table 2 the associated cardiovascular diseases.

Among the main cardiovascular complications, 6 pulmonary embolism, 4 deep venous thrombosis, 4 acute coronary syndrome, 5 strokes and 1 peripheral acute ischemia.

Sixty-five people have died $(24.9 \%)$. The main causes of deaths were ARDS (12.3\%) and sepsis and MOF (9.2\%).

Results: In Table 3 the comparison between demographic parameters of survivors and the deceased: the population that did not survive had an average age of almost 80 years, significantly higher than the group of living, and different comorbidities, mainly cardiovascular, that configured a greater degree of fragility. Treatment with RAAS inhibitors in the group of deceased has been more frequently suspended. Conclusions: The stop of therapy was found to be attributable to either erroneous beliefs borrowed from early Chinese reports or sepsis hypotension, and arterial hypertension or therapy with RAAS blockers cannot be considered risk factors due to the onset or to the manhood of Covid-19.

RAAS blockers certainly do not hurt, indeed it is hypothesized that their use is associated with protective effects on the prognosis of Covid-19, in line with what is stated in the Literature.

TABLE 1. DEMOGRAPHIC CHARACTERISTICS AT THE ONSET

$\begin{array}{ll}\text { Major Symptoms } & \text { Patients n.261 } \\ \text { fever } & 226(86.6 \%) \\ \text { dyspnea, tachypnea } & 122(46.7 \%) \\ \text { cough } & 116(44.4 \%) \\ \text { asthenia, anorexia } & 41(15.7 \%) \\ \text { diarrhea } & 27(10.3 \%) \\ \text { Minor Symptoms } & 13(5 \%) \\ \text { syncope } & 12(4.6 \%) \\ \text { lethargy } & 8(3.1 \%) \\ \text { arthralgia/myalgia } & 8(3.1 \%) \\ \text { nausea and vomit } & 8(3.1 \%) \\ \text { headache } & 8(3.1 \%) \\ \text { chest pain } & \end{array}$

\begin{tabular}{ll} 
TABLE 2. & \\
Associated cardiovascular diseases & Patients $\mathbf{n . 2 6 1}$ \\
\hline Hypertension & $161(61.7 \%)$ \\
Chronic heart failure & $57(21.8 \%)$ \\
Previous myocardial infarction or acute coronary syndrome & $31(11.8 \%)$ \\
Atrial fibrillation & $22(8.4 \%)$ \\
peripheral arteriopathy & $19(7.3 \%)$ \\
Previous stroke & $16(6.1 \%)$ \\
Chronic ischemic heart disease & $11(4.2 \%)$ \\
Other comorbidities & \\
Dyslipidemia & $100(38.3 \%)$ \\
Severe frailty & $69(26.4 \%)$ \\
Diabetes mellitus & $61(23.4 \%)$ \\
BMI > 30 kg/m2 & $58(22.2 \%)$ \\
Smoke & $47(18.0 \%)$ \\
COPD & $32(12.2 \%)$ \\
Active cancer & $26(10.0 \%)$ \\
CKD $\geq$ stage IV & $24(9.2 \%)$ \\
Hepatic cirrhosis & $7(2.7 \%)$ \\
Pulmonary hypertension & $4(1.5 \%)$
\end{tabular}

TABLE 3. COMPARISON BETWEEN DEMOGRAPHIC PARAMETERS OF SURVIVORS AND THE DECEASED

\begin{tabular}{llll|}
\hline Parameters & Alive n. 196 & Deceased n. 65 & $P$ \\
\hline Age & $65.9 \pm 14.2$ & $79.5 \pm 13.4$ & $<0.001$ \\
Male sex & $62.7 \%$ & $58.5 \%$ & NS \\
\hline Cardiovascular diseases & $15.8 \%$ & $38.5 \%$ & $<0.001$ \\
\hline Severe frailty & $7.6 \%$ & $41.5 \%$ & $<0.0001$ \\
\hline Visual score Ground Glass & $40.7 \%$ & $46.7 \%$ & NS \\
(\%) & $(5-90 \%)$ & $(10-80 \%)$ &
\end{tabular}

HEART RATE IN PATIENTS WITH SARS-COV-2 INFECTION: PREVALENCE OF HIGH VALUES AT DISCHARGE AND RELATIONSHIP WITH SEVERE DISEASE

Alessandro Maloberti ${ }^{1,2}$, Nicola $\mathrm{Ughi}^{3}$, Davida Paolo Bernasconi ${ }^{4}$, Marco Biolcati ${ }^{2}$, Valentina Giani ${ }^{2}$, Paola Rebora ${ }^{4}$, Enzo Grasso ${ }^{2}$, Iside Cartella ${ }^{2}$, Deborah Lenoci ${ }^{4}$, Francesca Del Gaudio ${ }^{4}$, Michela Algeri ${ }^{1}$, Sara Scarpellini ${ }^{1}$, Maria Grazia Valsecchi ${ }^{4}$, Claudio Rossetti $^{5}$, Oscar Massimiliano Epis ${ }^{4}$, Cristina Giannattasio ${ }^{1,2}$, On Behalf of the Covid-19 Niguarda Working Group

${ }^{1}$ Cardiology IV, “A.De Gasperis" Department, ASTT GOM Niguarda Ca' Granda, Milan, Italy, ${ }^{2}$ School of Medicine and Surgery,

University of Milano-Bicocca, Milan, Italy, ${ }^{3}$ Rheumatology, ASTT GOM Niguarda Ca' Granda, Milan, Italy, ${ }^{4}$ Center of Biostatistics for Clinical Epidemiology, School of Medicine and Surgery, University of Milano-Bicocca, Milan, Italy, ${ }^{5}$ Nuclear Medicine, ASTT GOM Niguarda Ca' Granda, Milan, Italy

Introduction: Despite the arrhythmic risk related to COronaVIrusrelated Disease (COVID) is still under evaluation, the most common arrhythmia associated with infection is sinus tachycardia. It is not known if high Heart Rate (HR) in COVID is simply a marker of an important systemic response to sepsis or if its persistence could be related to a long-term autonomic dysfunction.

Aim: The aim of our work is to assess the prevalence of elevated HR at discharge in patients hospitalized for COVID-19 and to evaluate the variables associated with it.

Methods: We enrolled 701 cases of SARS-CoV2 infection admitted in our hospital after February $21^{\text {st }}$ and discharged within July $23^{\text {rd }}$ 2020. We collected data on clinical history, vital signs, laboratory tests and pharmacological treatment. Severe disease was defined as the need for Intensive Care Unit (ICU) admission and/or mechanical ventilation.

Results: Median age was 59 years (IQR 49, 74), and male was the prevalent gender $(60.1 \%) .84 .7 \%$ of the subjects showed a SARSCoV-2 related pneumonia, and $13.4 \%$ resulted in a severe disease. Mean HR at admission was $90 \pm 18 \mathrm{bpm}$ with a mean variation of 10 bpm to discharge. Only $5.5 \%$ of subjects presented HR $>100 \mathrm{bpm}$ at discharge; they were younger (54-IQR 37-65 vs 60-IQR 50-74, years, $\mathrm{p}=0.004)$ and more frequently had a severe disease (36.8 vs $11.8 \%$, $\mathrm{p}$ $<0.001)$ with a longer hospital stay (21-IQR $10.5-34$ vs 15 IQR 9-24, days, $\mathrm{p}=0.036$ ) and a wider use of tocilizumab during their recovery (21.2 vs $9.9 \%, \mathrm{p}=0.055)$.

Furthermore, they presented higher HR (95-IQR 80-104 vs 88-IQR 77-101, bpm, $\mathrm{p}=0.037$ ) and body temperature (37.2-IQR 36.5-38 vs 36.7-IQR 36.1-37.6, $\left.{ }^{\circ} \mathrm{C}, \mathrm{p}=0.044\right)$ at admission as well as higher C-reactive protein (8.4-IQR 4.7-13.3 vs 4.3-IQR 1.4-9.3, mg/dL, p = 0.002). Significant predictors of discharge $\mathrm{HR}$ at multivariate model were admission HR ( $\beta=0.17,95 \%$ CI $0.11 ; 0.22, p<0.001)$, haemoglobin $(\beta=-0.64,95 \% \mathrm{CI}-1.19 ;-0.09, \mathrm{p}=0.023)$ and severe disease $(B=8.42,95 \%$ CI 5.39;11.45, $\mathrm{p}<0.001)$.

Conclusions: High HR at discharge in COVID-19 patients is not such a frequent problem, but when it occurs it seems strongly related to the evidence of a severe course of disease 


\section{THE ROLE OF TELEMEDICINE IN HYPERTENSION MANAGEMENT DURING SARS-COV-2 PANDEMIC: A PILOT STUDY IN A HYPERTENSION CENTRE}

Alberto Mazza ${ }^{1}$, Alice Bresolin ${ }^{2}$, Laura Schiavon ${ }^{1}$, Gioia Torin ${ }^{1}$, Ciro Rossetti $^{1}$, Rossana Redi ${ }^{1}$, Lorella Boreggio ${ }^{1}$, Marina Bellato ${ }^{1}$, Carla Destro $^{3}$, Stefano Cuppini ${ }^{4}$

${ }^{1}$ Unit of Hypertension and ESH Excellence Hypertension Centre, Department of Internal Medicine, Santa Maria della Misericordia General Hospital, Rovigo, Italy, ${ }^{2}$ School of Internal Medicine, University of Padua, Padua, Italy, ${ }^{3}$ Direction of the Hospital Function, AULSS5 Polesana, Santa Maria della Misericordia General Hospital, Rovigo, Italy, ${ }^{4}$ Internal Medicine Unit,

${ }^{2}$ Department of Internal Medicine, Santa Maria della Misericordia General Hospital, Rovigo, Italy

Introduction: With the emergence of the Sars-Cov-2 pandemic, Telemedicine (TM) has emerged as an important resource for improving the management of patients with chronic diseases such as arterial hypertension (HT).

Aim: To evaluate the efficacy and adherence to anti-hypertensive therapy in hypertensive outpatients with the TM service.

Methods: From January to April 2021, 51 hypertensive patients were analyzed (64\% females, average age $71.5 \pm 8.5$ years) and taking part in the Centro-Unico-Prenotazioni lists for hypertension visits. Patients had to have pathology exemption for HT (Codes 031-401 to 405) in order to guarantee their ticket-free service and were contacted by telephone to obtain consent to the TM service. If so, they filled a blood pressure (BP) diary, also noting the heart rate (HR) values of the 6 days before the doctor's telephone call. The diary was sent to them by e-mail or delivered to family members or caregivers. In addition to the antihypertensive therapy, the diary required to indicate the type of electronic BP devices used for BP measurement, as well as to report history of atrial fibrillation or use of oral anticoagulant drugs. Patients with systolic BP (SBP) and diastolic BP (DBP) values $<140 / 90 \mathrm{mmHg}$ were defined as having targeted BP values. Continuous variables were expressed as mean \pm standard deviation, while categorical variables were compared with Pearson's X2 test. The Student's T-test was used for the comparison of continuous variables by gender.

Results: SBP and DBP values were significantly increased in males than in females $(138.7 \pm 4.7$ vs. $134.3 \pm 12.4 \mathrm{mmHg}, \mathrm{p}<0.003$ and $81.7 \pm 3.0$ vs. $76.1 \pm 5.8 \mathrm{mmHg}, \mathrm{p}<0.001)$, while HR and number of antihypertensive drugs were not different by gender $(71.8 \pm 10.6$ vs. $71.3 \pm 8.4 \mathrm{bpm}$ and $2.5 \pm 0.8$ and $3.1 \pm 0.7$, NS). In $63.1 \%$ of subjects a target of BP was achieved and did not differ between males and females (61.1 vs. $64.0 \%$, NS). Among the antihypertensives, diuretics were the most frequently used $(70.6 \%)$, followed by calcium channel blockers $(64.7 \%)$, then ACE inhibitors (53\%), beta-blockers $(52.9 \%)$ and finally sartans $(35.3 \%)$; the prevalence of antihypertensive drugs were not different by gender. Anamnestic antihypertensive therapy remained unchanged.

ConclusionS: TM is effective in BP control, in monitoring adherence to therapy, in containing the costs of hypertension management and indirectly seems to have a potential reduction in waiting lists for HT visits.

\section{PULMONARY EMBOLISM AND LOCKDOWN: TREATMENT IN GREY ZONE}

Maria Lo Monaco, Francesco Cava, Ernesto Cristiano, Antonella Romaniello, Camillo Autore, Massimo Volpe

Department of Cardiovascular Disease, School of Medicine and Psychology, "Sapienza" University of Rome, Rome, Italy

Introduction: Pulmonary embolism (PE) represents a cardiovascular emergency in which treatment and diagnosis could be delayed because of the complex and non specific clinical manifestations.

In pre- Covid era, we observed less thrombotic complications. The main cause could be the reduction of physical activity and movements, during lockdown. Sedentary lifestyle and obesity can increase risk of trombotic complication in lower limbs which can cause pulmonary embolism.

Aim: Although anticoagulation is the standard treatment for PE, thrombolytic therapy has been considered an attractive alternative ${ }^{1-2}$ Methods: We describe a case of 79 years old woman, hemodynamically stable, with symptoms of dyspnoea. A dilated right ventricle (RV) and a moderate-severe tricuspid regurgitation (TR), due to massive PE, was detected by $2-\mathrm{D}$ transthoracic echocardiography (ETT).

Results: After 72 hours from thrombolytic therapy, ETT showed an improvement in RV size and TR jet, resulted in almost complete resolution of her PE.

Conclusions: Among patients with pulmonary embolism, including those who were hemodynamically stable with right ventricular dysfunction, thrombolytic therapy could be an attractive solution.

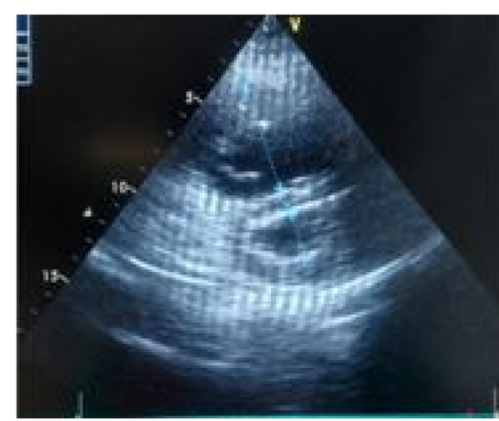

Pre-trombolisi

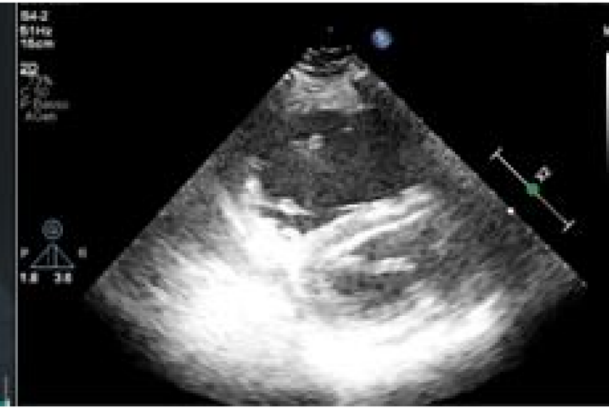

12h Post-trombolisi

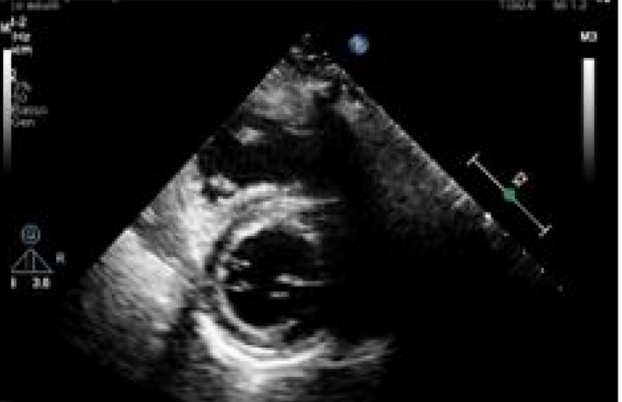

72h Post-trombolisi 


\section{ECHOCARDIOGRAPHIC ASPECTS IN PATIENTS WITH PREVIOUS SARS-COV-2 INFECTION}

Daniela Degli Esposti, Giulia Semprini, Arrigo FG Cicero, Stefano Bacchelli, Mario Soldati, Carmine Verde, Alessio Bragagni, Federica Fogacci, Alessandro Baracchi, Maddalena Veronesi, Claudio Borghi

Internal-Cardiovascular Medicine Department, Bologna University, Bologna, Italy

Introduction: Human corona-viruses impact on respiratory system had already well defined. Less known, conversely, is the impact on cardiovascular system, even if such virus has a well known myocardial tissue tropism, with cardiologic damage observed in about $20 \%$ of patients and a large range of clinical cardiologic manifestations.

Aim: To evaluate the impact of SARS-CoV-2 infection on cardiac structure and function, assessing echocardiographic aspects.

Methods: In a case-control study we compared 84 consecutive patients with previous SARS-CoV-2 infection, either with or without symptoms (CO: M/F 43/41, mean age $60.5 \pm 23.6$ years), with 84 patients who never had the infection (NoCO: M/F 43/41 mean age $60.3 \pm 23$ years, $\mathrm{p}=0.9$ ). Patients and controls received complete echocardiographic examination.

Results: 17 CO patients (20\%) had the infection without any clinical manifestations, while 49 patients (59\%) had clinical manifestations, mostly respiratory system disease. One patient died 4 weeks after severe respiratory disease partial recovery. No clinical information was available in 18 patients.

Basal characteristics were comparable in the two groups of patients, as well as the prevalence of comorbidity, such as hypertension, ischemic heart disease, diabetes, and atrial fibrillation.

As far as left ventricular echocardiographic aspects, $\mathrm{CO}$ patients showed a not significant trend towards a left ventricular concentric geometry (slightly higher values of wall thickness, left ventricular mass and relative wall thickness), and towards a relative worse left ventricular systolic function (FE CO $65 \pm 9 \%$, NoCO $67 \pm 6 \%, p=$ 0.06 ), while a slight but significant relative right ventricular dysfunction, assessed by tissue Doppler $\mathrm{S}$ wave reduction, was observed (S CO $13 \pm 3$, NoCO $14 \pm 3, \mathrm{p}=0.013$ ).

No differences between $\mathrm{CO}$ patients and controls was observed among diastolic function parameters.

Conclusions: SARS-Co-V-2 infection does not seem to be followed by overt left ventricular structural and functional damage, even if a trend towards concentric geometry and relative systolic dysfunction was observed.

Right ventricular systolic function was on the contrary significantly worse in $\mathrm{CO}$ patients respect to controls, despite only slightly and not significant differences in right ventricular systolic pressure, suggesting that the infection which mostly damages the respiratory system could however directly damage the right ventricle, the part of the heart which actually had the hemodynamic overload of the respiratory disease.

\section{ECHOCARDIOGRAPHIC LEFT VENTRICULAR GEOMETRY IN MEN AND WOMEN WITH PREVIOUS SARS-COV-2 INFECTION}

Daniela Degli Esposti, Giulia Semprini, Arrigo FG Cicero, Stefano Bacchelli, Mario Soldati, Alessio Bragagni, Federico Ruscelli, Federica Fogacci, Maddalena Veronesi, Claudio Borghi

Internal-Cardiovascular Medicine Department, Bologna University, Bologna, Italy

Introduction: SARS-CoV-2 infection, with its prevalent impact on respiratory system, is particularly ominous in hypertensive patients, mostly in those with bad blood pressure control.

Aim: To assess the impact of SARS-CoV-2 infection on cardiac structure and function, with particular attention to hypertensive organ damage.

Methods: In a case-control study we compared 84 consecutive patients with previous SARS-CoV-2 infection, either with or without symptoms (CO: M/F 43/41, mean age $60.5 \pm 23.6$ years), with 84 patients who never had the infection (NoCO: M/F 43/41 mean age $60.3 \pm 23$ years, $p=0.9)$. Patients and controls received complete echocardiographic examination.

Results: Basal characteristics were comparable in the two groups of patients, as well as the prevalence of comorbidity, in particular, hypertension was reported in $49 \%$ of CO patients and in $51 \%$ in NoCO ones.

As far as left ventricular echocardiographic aspects, $\mathrm{CO}$ patients showed a not significant trend towards higher values of wall thickness, left ventricular mass and relative wall thickness, and towards a worse left ventricular systolic function, therefore suggesting a higher prevalence of aspects usually considered as hypertensive left ventricular end organ damage.

When men and women are considered apart, we observed that while in men the differences were less evident, in women those global not significant differences became statistically significant. Women with previous COVID infection showed a more concentric left ventricular geometry, and worse left ventricular systolic function, that is left ventricular end organ damage (see table).

Conclusions: Hypertensive women, who, apart from COVID, usually have more hypertensive end organ damage, such as left ventricular hypertrophy, seem to emphasize such damage if exposed to COVID infection.

We cannot define if such end organ damage was present before the COVID infection or if it otherwise follows the COVID contact. In the first case it could be considered a sort of predisposition to the infection itself, while in the second one, it could be considered the persistence of a phlogistic condition with oedema localized in particular in myocardial tissue. 
TABLE: WOMEN WITH OR WITHOUT PREVIOUS SARS-COV-2 INFECTION

\begin{tabular}{|c|c|c|c|}
\hline $\begin{array}{l}\text { Echocardiographic } \\
\text { parameters }\end{array}$ & $\begin{array}{l}\text { Women with COVID-19 } \\
\text { (41 pz) }\end{array}$ & Controls (41 pz) & $P<0,05(1-2)$ \\
\hline IVSd & $1,02 \pm 0,27$ & $0,90 \pm 0,18$ & 0,019 \\
\hline LVIDd & $4,34 \pm 0,46$ & $4,30 \pm 0,40$ & 0,704 \\
\hline LVIDs & $2,74 \pm 0,31$ & $2,75 \pm 0,45$ & 0,916 \\
\hline LVPWd & $0,98 \pm 0,22$ & $0,88 \pm 0,15$ & 0,022 \\
\hline EDV Simpson $\mathrm{ml}$ & $70,47 \pm 21,72$ & $67,90 \pm 14,75$ & 0,534 \\
\hline ESV Simpson ml & $24,48 \pm 15,19$ & $21,03 \pm 5,83$ & 0,179 \\
\hline LV mas $g$ & $146,82 \pm 50,69$ & $125,68 \pm 39,69$ & 0,039 \\
\hline LV mas $\mathrm{g} / \mathrm{m}^{2}$ & $87,55 \pm 29,98$ & $77,39 \pm 23,19$ & 0,090 \\
\hline RWT & $0,46 \pm 0,14$ & $0,41 \pm 0,08$ & 0,059 \\
\hline EF Simpson \% & $65,91 \pm 9,75$ & $69,24 \pm 4,06$ & 0,047 \\
\hline FS & $37,79 \pm 5,10$ & $38,64 \pm 3,24$ & 0,620 \\
\hline $\mathrm{CO} \mathrm{L} / \mathrm{min}$ & $4,89 \pm 1,11$ & $5,31 \pm 1,15$ & 0,111 \\
\hline Septal $S_{1}$ & $7,30 \pm 1,85$ & $7,91 \pm 1,80$ & 0,140 \\
\hline Lateal $S_{1}$ & $8,84 \pm 2,21$ & $9,57 \pm 2,77$ & 0,195 \\
\hline Tricuspidal $\mathrm{S}_{1}$ & $12,65 \pm 2,53$ & $14,29 \pm 2,71$ & 0,006 \\
\hline
\end{tabular}

ROLE OF INTRACELLULAR PH IN THE SARSCOV-2 INFECTION: GITELMAN/BARTTER SYNDROMES, HUMAN MODEL OF POTENTIAL PROTECTION

Verdiana Ravarotto, Giovanni Bertoldi, Luca Sgarabotto, Lisa Gianesello, Laura Gobbi, Lorenzo A. Calò

Nephrology, Dialysis and Transplantation Unit, University of Padua, Padua, Italy

Introduction: ACE2, counter-regulatory of the renin-angiotensin system, serves both as protective toward oxidative stress and cardiovascular remodeling and as key entry for SARS-CoV-2. ACE2 is expressed as intracellular non-glycosylated and glycosylated isoform, being this latter accountable for the binding with SARS-CoV-2. After the binding, viruses uses proteases as cathepsin-L to entry the cells. Both glycosylation and cathepsin-L activity are $\mathrm{pH}$-dependent. Gitelman and Bartter syndromes (GSBS), rare genetic tubulopathies, are characterized by electrolytic alterations, activation of the reninangiotensin system, yet normo-hypotension, increased levels of ACE2 and metabolic alkalosis with likely increased intracellular $\mathrm{pH}$.
Aim: A survey in the first wave of COVID19 showed that none of GSBS patients located in the hotspots in Northern Italy has been infected. This prompted the rationale to exploit these patients as human model to investigate the binding and entry mechanisms of SARS-CoV-2.

Methods: Peripheral mononuclear cells isolated from 20 GSBS patients have been compared to those isolated from 15 heathy subjects. Western blot was used to assess ACE2 glycosylation and cathepsin-L activity has been evaluated by ELISA.

Results: Non-glycosylated ACE2 expression is significantly higher in GSBS compared to controls (GSBS $0.82 \pm 0.19$ d.u. vs $0.67 \pm 0.13$ d.u. $\mathrm{p}=0.01$ ), and, although not significant, the ratio between the two isoforms showed a lower glycosylation in GSBS compared to controls $(0.63+0.26$ vs $0.69+0.36, p=0.17)$. Cathepsin-L activity was significantly lower in GSBS $(3.90 \pm 1.13$ r.f.u. vs $5.31 \pm 0.8$ r.f.u. $\mathrm{p}=$ 0.0003).

Conclusions: These results demonstrate that GSBS's metabolic alkalosis, by increasing intracellular $\mathrm{pH}$, influences the glycosylation of proteins, specifically of ACE2, and the activity of cathepsin-L, supporting the hypothesis of a sort of endogenous protection of GSBS patients toward SARS-CoV-2 infection emerged in the survey. 


\section{CARDIOVASCULAR RISK SCORE DID NOT CORRELATE TO GAS EXCHANGE IN COVID19 PATIENTS}

Sebastiano Cicco $^{1}$, Chiara Mozzini ${ }^{2}$, Rossella Carella ${ }^{1}$, Giuditta De Fazio $^{1}$, Antonio Vacca $^{3}$, Christel Cariddo ${ }^{4}$, Angela Setti ${ }^{5}$, Fabrizio Pappagallo ${ }^{1}$, Antonio G. Solimando ${ }^{1}$, Roberto Ria ${ }^{1}$

${ }^{1}$ COVID section, Unit of Internal Medicine "Guido Baccelli", Department of Biomedical Sciences and Human Oncology, University of Bari, Bari, Italy; ${ }^{2}$ Department of Medicine, Section of Internal Medicine, Carlo Poma Hospital, Mantova, Italy; ${ }^{3}$ Division of Internal Medicine, Department of Medicine, Building 8, University of Udine, Udine, Italy; ${ }^{4}$ Department of Emergency and Organ transplants (DETO), Section of Anesthesiology and Resuscitation, Policlinico Hospital, University of Bari Aldo Moro, Bari, Italy; ${ }^{5}$ Department of Medicine, Section of Internal Medicine, University of Verona, Verona, Italy

Introduction: COVID-19 induce a robust systemic inflammation. Patients with cardiovascular disease (CVD) present an increased death risk. However, many efforts are spent to identify possible predictors of negative outcomes in order to have a more specific clinical setting. CVD score are useful tools in evaluation of risk of cardiovascular events

Aim: We evaluated oxygenation and characteristics in COVID-19 according to cardiovascular risk stratification performed using Framingham risk score (FRS) for cardiovascular disease.

Methods: We evaluated 155 COVID-19 patients (110 males and 45 females, aged $67.43 \pm 14.72$ yrs). All patients underwent to a complete physical examination, chest imaging, laboratory tests, and blood gas analysis at the time of diagnosis. Seventeen patients died (10 males and 7 females, aged $74.71 \pm 7.23$ yrs) while the remaining 138 patients (100 males and 38 females, aged $66.07 \pm 15.16$ yrs) were alive at discharge.

Results: Died patients have increased FRS compared to alive (27.37 \pm 5.03 vs $21.33 \pm 9.49, \mathrm{p}<0.05)$. No differences there were in $\mathrm{Hb}$, $\mathrm{C}$-reactive protein nor in d-dimers between the two groups. Compared to alive, died group presents a significant increase in white blood cells $(\mathrm{p}<0.05)$ and $\mathrm{d}$-dimers $(\mathrm{p}<0.05)$. No difference there were in $\mathrm{pCO} 2, \mathrm{SO} 2$, and in alveolar arteriolar oxygen difference $(\mathrm{A}-\mathrm{aDO} 2)$. On the contrary, in died patients there is an increased pO2 $(\mathrm{p}<0.05)$ and a decreased ratio between oxygen inspired and pO2 (P/F; p < $0.05)$. FRS presents a negative correlation to $\mathrm{P} / \mathrm{F}(\mathrm{r}-0.42, \mathrm{p}<0.05)$ in died while no correlation was found in alive. No other correlation has been found with blood gas parameters or in the phlogosis parameters evaluated in the two groups.

Conclusions: CVD may be considered as a major risk factor for death in COVID-19 patients. The increase risk relates to a reduced lung capacity but it is not related to alteration in gas exchange. Similarly, CV risk results independent from inflammatory state we found.

\section{ALTERATIONS OF BASAL SPIROMETRY IN LONG COVID PATIENTS. EFFECTS OF PHYSICAL ACTIVITY}

Pasquale Maria Parrella, Marco Rumolo, Roberto Bianco, Giada Annarumma, Eugenio di Vaia, Guido Iaccarino

Interdepartmental Center for Research in Arterial Hypertension and Related Pathologies, Federico II University of Naples
Introduction: The long-term sequelae of the COVID-19 pandemic, known as Long Covid (LC), are characterized by persistence, over 30 days, of fatigue, exercise intolerance, dyspnea and palpitations. Little is known about the pathophysiological mechanisms and the determinants of this syndrome.

Aim: Verify the impact of physical activity habits on LC, focusing on lung function, evaluated with traditional spirometry.

Methods: Out of 73 COVID-19 healed patients referred to the Sports Medicine Clinic of the Federico II University Hospital from December 2020 to May 2021 for sports fitness certification, we selected 19 patients aged between 40 and 65 years, with a mean age of $52.9 \pm 6.9$ years, of which 6 females and 13 males, with average body mass index (BMI) of $29.3 \pm 5.7$, negativized to the nasopharyngeal swab for at least 30 days. Inclusion criteria were COVID severity 2 , compliance to the spirometry test, absence of other conditions and medications that could interfere with spirometry. Patients were divided into sportsmen ( $\mathrm{S}, \mathrm{n}=7$, with a history of physical activity $>2 \mathrm{~h} /$ w) and non-sportsmen (NS, $\mathrm{n}=12$, with a sports history $<2 \mathrm{~h} / \mathrm{w}$, ) and subjected to traditional spirometry (Spirostick blue, Gerotherm), for the evaluation of forced vital capacity (FVC), tidal volume (CV), maximum expiratory volume in the first second (FEV1), Tiffenau index and maximum voluntary ventilation (MVV) calculated with reference to the expected theoretical values.

Results: Considering the population as whole, we observed the increase in FEV1/FVC compared to the theoretical value $(87.9 \pm 1.5$ vs. $79.9 \pm 0.6 \%, p<0.001)$. Dicotomising for sport habit, this difference was confirmed in both subgroups (S: $89.1 \pm 2.2$ vs. $79.4 \pm$ $1.0 \%, \mathrm{p}<0.001$; NS: $87.2 \pm 2.0$ vs $80.2 \pm 0.8 \%, \mathrm{p}<0.001)$. This increase is due to the significant reduction in FVC in NS patients (3.7 \pm 0.2 vs. $4.0 \pm 0.2 \mathrm{~L}, \mathrm{p}<0.05)$ but not in S patients $(4.2 \pm 0.4$ vs. 4.2 $\pm 0.3 \mathrm{~L}, \mathrm{~ns})$. Finally, CV reduction is also observed in NS patients $(3.6 \pm 0.2$ vs. $4.0 \pm 0.2 \mathrm{~L}, \mathrm{p}<0.05)$, but not in $\mathrm{S}$ patients $(4.5 \pm 0,3$ vs. $4.3 \pm 0.3 \mathrm{~L}$, ns).

Conclusion: LC is characterized by remodeling of lung function that is still observable over 1 month after negative swab. However, in NS patients, this is accompanied by structural changes, which are prevented in patients with a sport habit. Our data support the importance of physical activity in the prevention and treatment of LC outcomes.

\section{AGE AND COMORBIDITIES PREDICT THE WORST PROGNOSIS IN PATIENTS OF THE SECOND AND THIRD WAVE OF COVID- 19 PANDEMICS}

Guido Iaccarino ${ }^{1}$, Massimo Salvetti ${ }^{2}$, Leonardo Sechi $^{3}$, Claudio Borghi ${ }^{4}$, Massimo Volpe ${ }^{5}$, Maria Lorenza Muiesan ${ }^{2}$, Claudio Ferri ${ }^{6}$, Guido Grassi $^{7}$

${ }^{1}$ CIRIAPA, Federico II University, Naples, Italy, ${ }^{2}$ Department of Clinical and Experimental Sciences, University of Brescia, Brescia, Italy, ${ }^{3}$ Department of Area Medica, University of Udine, Udine, Italy, ${ }^{4}$ Department of Medical and Surgical Sciences, University of Bologna, Italy, ${ }^{5}$ Department of Medicina Clinica e Molecolare University of Roma La Sapienza, Italy, ${ }^{6}$ Department of Medicina Clinica, Sanità Pubblica, Scienze della Vita e dell'Ambiente, University of L'Aquila, Italy, ${ }^{7}$ Department of Medicine and Surgery, University of Milano, Bicocca, Italy

Introduction: We recently reported that during the first wave of COVID-19, age and multimorbidity were the major determinants of mortality. 
Aim: In this study we extend the observation also to patients of the second and third pandemic waves, with the intent to verify eventual differences between the determinants of mortality.

Methods: 1734 records were recorded (male 56, \%, $67.8 \pm 0.4$ years). At least 1 pre-existing comorbidity was observed in $79.3 \%$ of patients, and hypertension was the most represented (60\%). 148 deaths were recorded $(8.5 \%$, mean age $82.9 \pm 0.9$ years). In nonsurvivors, on binary correlation analysis, hypertension, diabetes, chronic obstructive pulmonary disease (COPD), chronic kidney disease (CKD), coronary artery disease (CAD), and heart failure (HF) were more represented with respect to the survivors.

Results: Similarly to what previously observed, in this new population, the Charlson Co-Morbidity Index (CCI), which combines age and comorbidities, was significantly higher in non-survivors than in survivors $(6.1 \pm 0.16$ vs $4.4 \pm 0.05, \mathrm{p}<0.001)$ and predicts mortality among COVID-19 patients $(0.433), \mathrm{p}<0.01)$. Among the chronic treatments taken by patients for their conditions, diuretics $(\mathrm{B}=0.959$; $\mathrm{p}<0.001)$ are associated with a worse prognosis, while ACE inhibitors $(\mathrm{B}=-0.547, \mathrm{p}<0.001)$ are protective. These associations continue to be significant even in multivariate analysis (Table 1), where however, the negative role of diuretic mortality is no longer evident.

Conclusions: Our data confirm that age and comorbidities predict death in covid-19 disease but suggest the protective role of ACE inhibitors. This data will be confirmed in the cumulative analysis of first, second and third wave patients.

\section{Heart}

\section{A NOVEL APPROACH TO LEFT VENTRICULAR FILLING PRESSURE ASSESSMENT: THE ROLE OF HEMODYNAMIC FORCES ANALYSIS}

Lorenzo Airale ${ }^{1}$, Fabrizio Vallelonga ${ }^{1}$, Tommaso Forni ${ }^{1}$, Dario Leone $^{1}$, Anna Astarita ${ }^{1}$, Giulia Mingrone ${ }^{1}$, Marco Cesareo ${ }^{1}$, Carlo Giordana $^{1}$, Pierluigi Omedè ${ }^{2}$, Claudio Moretti ${ }^{2}$, Franco Veglio ${ }^{1}$, Gianni Pedrizzetti ${ }^{3}$, Alberto Milan ${ }^{1}$

${ }^{1}$ Internal Medicine and Hypertension Division, Department of Medical Sciences, AOU "Città della Salute e della Scienza” Hospital, University of Turin, Turin, Italy, ${ }^{2}$ Hemodynamic Laboratory, Department of Medical Sciences, AOU "Città della Salute e della Scienza" Hospital, University of Turin, Turin, Italy, ${ }^{3}$ Department of Engineering and Architecture, University of Trieste, Trieste, Italy

Introduction: diastolic function in patients with heart failure (HF) is usually impaired, resulting in increased left ventricular (LV) filling pressures, whose gold standard assessment is right heart catheterization (RHC). Hemodynamic forces (HDFs) analysis is a novel echocardiographic tool, providing an original approach to cardiac function assessment through the speckle-tracking technology.

Aim: to evaluate the use of HDFs, both alone and included in a new predictive model, as a potential novel diagnostic tool of the diastolic function.

MethodS: HDFs analysis was retrospectively performed in 67 patients enrolled in the "Right1 study". All patients underwent RHC and echocardiography up to 2 hours apart. Increased LV filling pressure (ILFP) was defined as pulmonary capillary wedge pressure (PCWP) $\geq 15 \mathrm{mmHg}$.

Results: 33 patients out of $67(49.2 \%)$ showed ILFP at RHC. Diastolic longitudinal force (DLF) was associated with the presence of ILFP $(\mathrm{OR}=0.84$ [0.70;0.99], $\mathrm{p}=0.046)$. The PCWP prediction score we built including DLF, ejection fraction, left atrial enlargement and e' septal showed an AUC of 0.83 [0.76-0.89], with an optimal internal validation. When applied to our population, the score showed a sensitivity of $72.7 \%$ and a specificity of $85.3 \%$, which became $66.7 \%$ and $94.4 \%$ respectively, when applied to patients classified with "indeterminate diastolic function" according to current recommendations.

Conclusions: HDFs analysis could be an additional useful tool in diastolic function assessment. A scoring system including HDFs might improve echocardiographic accuracy in estimating LV filling pressures. Further carefully designed studies could be useful to clarify the additional value of this new technology.

\section{URIC ACID IS ASSOCIATED WITH ACUTE HEART FAILURE AND CARDIOGENIC SHOCK AT PRESENTATION IN ACUTE CORONARY SYNDROMES PATIENTS}

Alessandro Maloberti ${ }^{1,2}$, Paola Rebora ${ }^{3}$, Marco Centola ${ }^{4}$, MassiNuccia Morici ${ }^{5}$, Alice Sacco ${ }^{5}$, Giuseppe Occhino ${ }^{3}$, Giovanna Viola $^{5}$, Jacopo Oreglia ${ }^{5}$, Diego Castini ${ }^{4}$, Simone Persampieri ${ }^{4}$, Ludovico Sabatelli ${ }^{4}$, Giulia Ferrante ${ }^{4}$, Stefano Lucreziotti ${ }^{4}$, Stefano Carugo $^{4}$, Maria Grazia Valsecchi ${ }^{3}$, Fabrizio Oliva ${ }^{5}$, Cristina Giannattasio $^{1,2}$

${ }^{1}$ Cardiology 4, Cardio Center, ASST GOM Niguarda, Milan, Italy, ${ }^{2}$ School of Medicine and Surgery, Milano-Bicocca University, Milan, Italy, ${ }^{3}$ Bicocca Center of Bioinformatics, Biostatistics and Bioimaging (B4 center), School of Medicine and Surgery, University of Milano-Bicocca, Monza, Italy, ${ }^{4}$ Division of Cardiology, ASST Santi Paolo Carlo, Department of Cardio-Respiratory Disease, University of Milan, Milan, Italy, ${ }^{5}$ Cardiology 1 , Cardio Center, ASST GOM Niguarda, Milan, Italy

Introduction: We focused on the role of Uric Acid (UA) as a possible determinant of Heart Failure (HF) related issues in Acute Coronary Syndromes (ACS) patients.

Aim: Main outcomes were acute HF and cardiogenic shock at admission, secondary outcomes were the need of Non Invasive Ventilation (NIV) use and the admission Left Ventricular Ejection Fraction (LVEF).

Methods: We consecutively enrolled 1269 ACS patients admitted to the cardiological Intensive Care Unit of the Niguarda and San Paolo hospitals (Milan, Italy) from June 2016 to June 2019. Hyperuricemia was defined as a value higher than $6 \mathrm{mg} / \mathrm{dL}$ for females and $7 \mathrm{mg} / \mathrm{dL}$ for males.

Results: All the evaluated outcomes occurred more frequently in the hyperuricemic subjects $(\mathrm{n}=292)$ : acute HF 35.8 vs $11.1 \%(\mathrm{p}<$ 0.0001 ), cardiogenic shock 10 vs $3.1 \%$ ( $p<0.0001$ ), NIV 24.1 vs $5.1 \%(\mathrm{p}<0.0001)$ with lower admission LVEF $(42.9 \pm 12.8$ vs 49.6 $\pm 9.9, \mathrm{p}<0.0001)$. By multivariable analyses, UA was confirmed to be significantly associated with all the outcomes with the following Odds Ratio (OR): acute HF OR $=1.119 ; 95 \%$ C.I. 1.019;1.229; cardiogenic shock OR $=1.157 ; 95 \%$ C.I. $1.001 ; 1.337$; NIV use OR = 1.208; 95\% C.I. $1.078 ; 1.354$; LVEF $\beta=-0.999 ; 95 \%$ C.I. -1.413 ; 0.586 .

Conclusion: The main result of our study was the finding of a significant association between UA and acute HF, cardiogenic shock, NIV use and LVEF. Due to the cross-sectional nature of our study no definite answer on the direction of these relationship can be drawn and further longitudinal study on UA changes over time during an ACS hospitalization are needed. 


\section{IMPACT OF THE INCREASE OF LEFT VENTRICULAR MASS ON THE RISK OF ALL- CAUSE AND CARDIOVASCULAR MORTALITY: A PROSPECTIVE COHORT STUDY}

\author{
Jennifer Vanoli ${ }^{2}$, Raffaella Dell'Oro ${ }^{1,2}$, Michele Bombelli ${ }^{2,3}$, Fosca \\ Quarti-Trevano $^{1,2}$, Rita Facchetti ${ }^{2}$, Giuseppe Mancia ${ }^{2}$, Guido Grassi \\ $\underset{1,2}{2}$
}

\author{
${ }^{1}$ Division of Medical Clinic, San Gerardo Hospital, Monza, Italy, \\ ${ }^{2}$ University of Milano-Bicocca, Milan, Italy, ${ }^{3}$ Division of Internal \\ Medicine, Desio Hospital, Desio, Italy
}

Introduction: Left ventricular hypertrophy $(\mathrm{LVH})$ is a known independent predictor of nonfatal and fatal cardiovascular (CV) events as well as all-cause death. While the beneficial effect of the regression of LVH by treatment is well known, the association between its progression and cardiovascular risk has been the subject of only few studies.

Aim: To follow-up the increase of LVM over time and assess its prognostic impact in term of CV and all-cause mortality.

Methods: We studied by echocardiography subjects enrolled in the PAMELA study (Pressioni Arteriose Monitorate E Loro Associazioni), a cohort recruited to be representative of the general population. 1, 114 subjects have been followed up for an average of 16 years with LVM measurement by echocardiogram at the baseline and 10 years after.

Results: Mean changes of LVM from baseline ( $\triangle \mathrm{LVM}$ ) were $26.94 \pm$ $37.22 \mathrm{~g}$ in absolute value, and $13.5 \pm 20.42 \mathrm{~g} / \mathrm{m}^{2}$ and $7.49 \pm 9.94 \mathrm{~g} / \mathrm{h}^{2.7}$ in values indexed respectively for BSA and height ${ }^{2.7}$. In the whole population 59 deaths were recorded including 19 of proven cardiovascular origin. We observed an association between the increase of LVM and the risk of cardiovascular death, that was even more evident in those subjects without $\mathrm{LVH}$ at the baseline $(\mathrm{HR}=1.031, \mathrm{p}=$ 0.0005 for each unite increase in $g ; H R=1.061, p=0.0002$ for each unite increase in $\mathrm{g} / \mathrm{m}^{2} ; \mathrm{HR}=1.141, \mathrm{p}<0.0001$ for each unite increase in $\left.1 \mathrm{~g} / \mathrm{h}^{2.7}\right)$. This association hold true also after adjustment for principal confounding factors such as age, sex, previous cardiovascular nonfatal event, BMI, ambulatory SBP, antihypertensive treatment and basal LVM. Progression of LVM was also related to the increase risk of all-cause death in subjects without LVH at the baseline independently of other risk factors.

Conclusions: Our data suggest performing, in subjects exposed to the risk of progression or development of $\mathrm{LVH}$, echocardiographic monitoring of LVM for prognostic stratification and effective and prompt therapeutic intervention.

\section{PREVALENCE AND PREDICTORS OF SUBCLINICAL ATRIAL FIBRILLATION IN OLDER ADULTS HOSPITALIZED FOR ACUTE MEDICAL CONDITIONS}

Francesco Spannella ${ }^{1,2}$, Federicco Giulietti ${ }^{1,2}$, Lorenzo Pimpin ${ }^{3}$, Francesca Elena Lombardi ${ }^{1,2}$, Serena $\operatorname{Re}^{1,2}$, Paola Schiavi ${ }^{1,2}$, Gina Dragano $^{1}$, Roberto Antonicelli ${ }^{3}$, Riccardo Sarzani ${ }^{1,2}$

${ }^{1}$ Internal Medicine and Geriatrics, IRCCS INRCA, Ancona, Italy, ${ }^{2}$ Department of Clinical and Molecular Sciences, University "Politecnica delle Marche", Ancona, Italy, ${ }^{3}$ Cardiology Unit, IRCCS INRCA, Ancona, Italy

Introduction: Subclinical atrial fibrillation (SCAF) is associated with an increased risk of clinical AF, major cardiovascular events and death. Most of the published studies are focused on high-risk adult populations, such as patients with implantable cardiac devices. Shortterm evidence on SCAF in older population is scarce, especially in the hospital setting.

Aim: We performed a cross-sectional study on 60 multimorbid older consecutive patients (aged 80+) admitted to an Internal Medicine and Geriatrics Unit for acute medical diseases with no history of AF, in order to investigate prevalence and predictors of SCAF.

Methods: Portable 3-channel ECG monitoring was placed within the first 12 hours from admission and ECG recording lasted for 5 days. Results: Mean age: $85.7 \pm 4.9$ years. Female prevalence: $58.3 \%$. High burden of comorbidities: $87.9 \%$. Hypertension was present in $83.1 \%$ of patients. All enrolled patients had $\mathrm{CHA}_{2} \mathrm{DS}_{2}$-VASc score $\geq$ 3. SCAF was detected in 16 patients $(26.7 \%)$ and 11 patients $(18.4 \%)$ had at least a SCAF episode lasting 6 minutes or longer. No clinical, laboratory and echocardiographic parameters predicted SCAF. Patients with $\geq 2004$ supraventricular ectopic beats/24h (SVEBs/24h) had a 6-fold higher prevalence of SCAF than patients with $<411$ SVEBs/24h $(p=0.038)$. Time to first SCAF episode was within 3 days of ECG recording in all enrolled patients.

Conclusions: SCAF is highly prevalent in the older adults hospitalized for acute medical conditions, regardless of the history of hypertension. If confirmed, although there are still uncertainties about the prognostic value of SCAF, our preliminary findings may affect clinical management of these patients. Our study could foster larger multicenter studies in similar settings.

\section{ECHOCARDIOGRAPHIC AND HEMODYAMIC ASPECTS IN DIFFERENT HYPERTENSIVE DISORDERS OF PREGNANCY}

Daniela Degli Esposti, Marta Mariani, Arrigo FG Cicero, Stefano Bacchelli, Giacomo Nicolini, Eugenia Ianniello, Mario Soldati, Federica Fogacci, Maddalena Veronesi, Claudio Borghi

Internal-Cardiovascular Medicine Department, Bologna University, Bologna-Italy

Introduction: Hypertensive disorders in pregnancy comprehend different conditions.

Aim: To evaluate maternal echocardiographic aspects in different kind of hypertensive diseases during pregnancy, to assess if echocardiographic characteristics could help in identify patients who will actually develop more ominous conditions, such as preeclampsia, and who will therefore need a more strict follow-up during their pregnancy.

Methods: We studied, with clinical and echocardiographic evaluation during various trimesters of pregnancy, 221 women with highrisk pregnancy (previous preeclampsia, PE, chronic or gestational hypertension, $\mathrm{CGH}$, diabetes, or immunological diseases), with a total number of 550 clinical/echocardiographic exams. Women were divided in groups according to complications occurred during pregnancy or at delivery time.

Results: 57 women did not develop any complications (NC) (mean age $36.2 \pm 5.9$ years), 64 had PE (age $36.3 \pm 5.1$ years), 89 CGH (age $37.7 \pm 5.3$ years $), 11 \mathrm{D}(36.2 \pm 5.8$ years; $\mathrm{p}=0.02)$. Diabetes was considered among "hypertensive" complications because of its high cardiovascular risk profile shared with hypertension.

Among PE women there were more prevalence of first pregnancy, while CGH and D showed higher body weight and body mass index.

PE and CGH had higher blood pressure (NC 114/67 $\pm 11 / 8$, PE $126 / 8 \pm 16 / 9, \operatorname{cgh} 126 / 78 \pm 14 / 10, \mathrm{D} 115 / 71 \pm 11 / 9 ; \mathrm{p}=0.00)$, and peripheral vascular resistances (NC $1182.8 \pm 284.4$, PE $1529.8 \pm$ 324.1 , CGH $1319.8 \pm 316.9$, D $1228.9 \pm 288.9 ; \mathrm{p}=0.00)$. 
As regard as echocardiographic parameters, PE and CGH showed higher wall thickness, left ventricular mass, and relative wall thickness (see table), with a trend toward a more concentric left ventricular geometry, suggesting in these women a particular trend toward the development of hypertensive end organ damage, considering the relative short time in which hypertension was present, and in which it could have therefore caused its damages.

Moreover, PE showed worse left ventricular contractility parameters respect to other groups (see table).

Conclusions: PE could be considered an accelerating factor for hypertensive damage on left ventricular function. However, we cannot exclude that PE occurs just in women with an intrinsic predisposition toward a particularly vulnerable cardiovascular risk profile.

TABLE
CARDIAC PRESSURE OVERLOAD INDUCES THE EXPRESSION OF PLACENTAL GROWTH FACTOR IN THE SPLEEN WHERE IT IS NECESSARY TO ALLOW THE RECRUITMENT OF REPARATIVE MACROPHAGES IN THE LEFT VENTRICLE

${ }^{1}$ Sara Perrotta, ${ }^{2}$ Raimondo Carnevale, ${ }^{2}$ Fabio Pallante, ${ }^{2}$ Francesco Mastroiacovo, ${ }^{2}$ Giuseppe Cifelli, ${ }^{1,2}$ Giuseppe Lembo, ${ }^{1,2}$ Daniela Carnevale

${ }^{1}$ Department of Molecular Medicine, "Sapienza" University of Rome, Italy, ${ }^{2}$ Department of Angiocardioneurology and Translational Medicine, IRCCS Neuromed, Pozzilli (IS), Italy

\begin{tabular}{|l|c|c|c|c|c|}
\hline $\begin{array}{l}\text { Echo- } \\
\text { parameter }\end{array}$ & $\begin{array}{l}\text { Not } \\
\text { complicated } \\
\text { pregnancy } \\
(57)\end{array}$ & $\begin{array}{l}\text { Preeclampsia } \\
(64)\end{array}$ & $\begin{array}{l}\text { Chronic/gesta } \\
\text { tional } \\
\text { hypertension } \\
(89)\end{array}$ & Diabetes (11) & P $<0,05$ \\
\cline { 1 - 4 } IVSd & $0,85 \pm 0,08$ & $0,91 \pm 0,13$ & $0,91 \pm 0,13$ & $0,88 \pm 0,08$ & 0,00 \\
\hline LVMg & $131,3 \pm 21,8$ & $143,8 \pm 43,5$ & $146,2 \pm 37,3$ & $136,2 \pm 21,9$ & 0,00 \\
\hline LV mass(g2.7) & $35,5 \pm 6,2$ & $37,7 \pm 10,7$ & $38,5 \pm 9,4$ & $37,8 \pm 5,9$ & 0,01 \\
\hline LV mass(gm2) & $74,5 \pm 10,4$ & $79,5 \pm 18,1$ & $79,5 \pm 16,0$ & $74,8 \pm 10,8$ & 0,01 \\
\hline RWT & $0,37 \pm 0,04$ & $0,40 \pm 0,1$ & $0,39 \pm 0,1$ & $0,37 \pm 0,1$ & 0,00 \\
\hline CO & $5,8 \pm 1,2$ & $5,2 \pm 1,2$ & $5,9 \pm 1,3$ & $5,8 \pm 1,1$ & 0,01 \\
\hline $\begin{array}{l}\text { EF (Simpson) } \\
\text { \% }\end{array}$ & $70,1 \pm 4,3$ & $68,1 \pm 5,9$ & $70,3 \pm 6,1$ & $71,5 \pm 5,4$ & 0,00 \\
\hline LVPWd & $0,8 \pm 0,1$ & $0,9 \pm 0,1$ & $0,9 \pm 0,1$ & $0,9 \pm 0,1$ & 0,00 \\
\hline FS \% & $39,3 \pm 5,3$ & $38,5 \pm 4,7$ & $39,6 \pm 3,9$ & $41,0 \pm 3,3$ & 0,02 \\
\hline Sept S1 & $8,6 \pm 1,1$ & $7,8 \pm 1,1$ & $8,1 \pm 1,3$ & $8,3 \pm 1,0$ & 0,00 \\
\hline & & & & & \\
\hline
\end{tabular}

Introduction: Cardiac remodeling to pressure overload is a process of adaptation of the left ventricle (LV) that involves immune cells. We found that Placental Growth Factor (PIGF), a growth factor of the VEGF family, is necessary for the adaptive remodeling to pressure overload induced by transverse aortic coartaction (TAC).

Aim: Aim of this study was to investigate the role of PIGF as an immunomodulator in response to TAC. 
Methods: We analyzed the cardiac remodeling by echocardiographic analysis and the cardiac monocyte/macrophage populations by flow cytometry to discriminate resident and recruited macrophages.

Results: After TAC, WT mice, but not the PIGF KO mice, showed a significant infiltrate of non-resident macrophages, suggesting that PIGF is involved in the recruitment of immune cells. Since TAC induces the expression of PlGF in the spleen, we investigated the potential role of the spleen as a reservoir of myeloid cells with adaptive/reparative functions. We subjected to TAC splenectomized mice that exhibited early heart failure (HFrEF) and reduced recruitment of monocytes/macrophages in the LV. To investigate the potential involvement of Neuropilin-1 (NRP1) as a receptor of PIGF in the recruitment of monocytes/macrophages in $\mathrm{LV}$, we subjected to TAC mice with selective deletion of NRP1 in myeloid lineage. We observed HFrEF similar to splenectomized mice and PIGF KO mice. To characterize the contribution of adaptive/reparative macrophages, characterized by the expression of the $\mathrm{CX}_{3} \mathrm{CR} 1$ receptor, and of the pro-inflammatory monocytes expressing CCR2, we subjected $\mathrm{CX}_{3} \mathrm{CR} 1 \mathrm{KO}$ and CCR2 KO mice to TAC. While CCR2 KO mice showed adaptive remodeling such as WT, $\mathrm{CX}_{3} \mathrm{CR} 1 \mathrm{KO}$ mice developed HFrEF similar to splenectomized mice and PIGF KO mice.

Conclusions: Our results indicate that pressure overload induces PlGF in the spleen and the PlGF/Nrp1 pathway is necessary to promote the recruitment of adaptive/reparative macrophages in $\mathrm{LV}$ contributing to the establishment of an adaptive cardiac remodeling to pressure overload. echocardiogram including Doppler and Tissue Doppler recordings. Symmetric and Asymmetric AASI were obtained by blood pressure recordings. Diastolic function was evaluated by measuring Early (E) and Late (A) peak of mitral inflow velocities, Deceleration Time (DT) of early filling and Isovolumetric Relaxation Time (IVRT) obtained from pulsed-wave Doppler. Tissue Doppler Imaging (TDI) was performed for measuring the peak velocities in systole $(\mathrm{Sm})$, early $(\mathrm{Em})$ and late $(\mathrm{Am})$ diastole and $\mathrm{E} / \mathrm{Em}$, placing the sample volume on the lateral mitral annulus.

Results: According to univariate analysis, the strongest correlations were observed between left ventricular diastolic function parameters (E/Em and A) and symmetrical and asymmetric AASI (see Table). At multivariate analysis, the association between symmetric AASI and A remained statistically significant even after adjustment for potential confounding factors including age, Left Ventricular Mass normalized for height, sex, BMI and systolic blood pressure, while the association between symmetric-AASI and E/Em lost statistical significance when PP $24 \mathrm{~h}$ was inserted into the model

Conclusions: Our results showed an independent association between arterial stiffness indices and left ventricular diastolic function parameters.

\begin{tabular}{|c|c|c|c|c|c|c|c|c|c|c|}
\hline \multicolumn{1}{|l|}{ Table } \\
\hline AASI & r & NS & $0.37^{* * *}$ & $-0.18^{*}$ & $0.27^{* *}$ & NS & $-0.23^{* *}$ & $-0.24^{* * *}$ & NS & $0.33^{* * *}$ \\
\hline s-AASI & $r$ & NS & $0.40^{* * *}$ & $-0.19^{*}$ & $0.14^{*}$ & NS & $-0.19^{*}$ & $-0.26^{* * *}$ & NS & $0.36^{* * *}$ \\
\hline$* p<.05, * *$ & & & & & & & & \\
\hline
\end{tabular}

\section{RELATIONSHIP BETWEEN SYMMETRIC AND ASYMMETRIC AMBULATORY ARTERIAL STIFFNESS INDEXES AND LEFT VENTRICULAR DIASTOLIC FUNCTION PARAMETERS IN HYPERTENSIVE PATIENTS}

Ettore Mancia ${ }^{1}$, Emilio Nardi ${ }^{2}$, Alessandra Sorce ${ }^{1}$, Dario Altieri ${ }^{1}$, Alessandra Ferotti ${ }^{1}$, Caterina Carollo ${ }^{1}$, Santina Cottone ${ }^{1}$

${ }^{1}$ ESH Hypertension Excellence Centre and Nephrology UnitDepartment of Health Promotion, Mother and Child Care, Internal Medicine and Medical Specialties, University of Palermo, Palermo, Italy, ${ }^{2}$ Department of Health Promotion, Mother and Child Care, Internal Medicine and Medical Specialties-Unit of Internal Medicine, University of Palermo, Palermo, Italy

Introduction: AASI (Ambulatory arterial stiffness indices) has been associated with an increased risk of stroke. Little is known about the relationship of AASI with left ventricular diastolic function.

Aim: The aim of our study was to evaluate the relationship between left ventricular diastolic function assessed by echocardiography Doppler and the so-called "Ambulatory" arterial stiffness indices (AASI).

Methods: We enrolled 222 patients, of which $60 \%$ were male, (mean age $54 \pm 15$ years) not pharmacologically treated and with normal renal function. All the patients underwent ambulatory blood pressure monitoring (ABPM) using an oscillometer device and

\section{CARDIOVASCULAR RISK STRATIFICATION IN MULTIPLE MYELOMA PATIENTS UNDERGOING CARFILZOMIB: THE ROLE OF THE LEFT VENTRICULAR HAEMODYNAMIC FORCES}

Anna Astarita, Giulia Mingrone, Anna Colomba, Lorenzo Airale, Cinzia Catarinella, Marco Cesareo, Fabrizio Vallelonga, Dario Leone, Giulia Bruno, Franco Veglio, Alberto Milan

Hypertension Unit, Department of Medical Sciences, Division of Internal Medicine, AO "Città della Salute e della Scienza" University Hospital, Turin, Italy

Introduction: Carfilzomib (CFZ) therapy is linked to cardiotoxicity development in patients with multiple myeloma (MM). The role of echocardiography in the baseline assessment is pivotal, however are lacking parameters of subclinical ventricular damage, potentially involved in cardiovascular adverse events (CVAEs) development. The evaluation of the left ventricle haemodynamic forces (HFLV), through a new echocardiographic software using the speckle-tracking methodology, could be useful in identification of subclinical ventricle damage.

Aim: The aim of this prospective study was to investigate the role of HFLV at baseline in predicting CVAEs.

Methods: 40 patients with MM candidates for CFZ therapy were consecutively enrolled. They underwent to a baseline assessment 
including Echocardiography TT (ETT) and the study of HFLV (Software Diogenes, Italy). During the follow-up, the incidence of CVAEs was detected.

Results: Median age was $67 \pm 7.8$ years old, $50 \%$ males. During the follow-up, $57.5 \%$ of patients developed CVAEs. At ETT, there were no differences in left ventricular mass and ejection fraction between no CVAEs and CVAEs groups. HFLV analysis showed reduced longitudinal and later-septal haemodynamic forces (during the cardiac cycle and systole) in patients with CVAEs $(\mathrm{p}<0.005)$, demonstrated a potential ventricular disfunction. Indeed, the LV work during the systolic acceleration phase was reduced in patients with CVAEs. Through the ROC curve analysis, we investigated potential cut-off of each haemodynamic force, all predictors of CVAEs (OR > 4).

Conclusions: HFLV could be more sensible parameters of subclinical ventricular damage, compared to morphologic ones. Their application in clinical practice could be useful for the correct cardiovascular risk estimation at baseline, identifying the higher risk patients for CVAEs during CFZ therapy.
Epidemiology and Clinical Aspects

\section{PREVALENCE OF TARGET ORGAN DAMAGE IN SUBJECTS WITH HIGH-NORMAL BLOOD PRESSURE WITHOUT KNOWN CARDIOVASCULAR AND KIDNEY DISEASE}

Giacomo Ruzzenenti ${ }^{1,2}$, Alessandro Maloberti ${ }^{1,2}$, Paola Rebora ${ }^{3}$, Giuseppe Occhino ${ }^{3}$, Marta Alloni ${ }^{1}$, Francesco Musca ${ }^{2}$, Oriana Belli ${ }^{2}$, Francesca Spano ${ }^{2}$, Gloria Maria Santambrogio ${ }^{2}$, Francesca Casadei ${ }^{2}$, Antonella $\mathrm{Moreo}^{2}$, Maria Grazia Valsecchi ${ }^{3}$, Cristina Giannattasio ${ }^{1,2}$

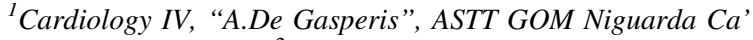
Granda, Milan, Italy, ${ }^{2}$ University of Milano Bicocca, Milan, Italy, ${ }^{3}$ Center for Bioinformatics, Biostatistics and Bioimaging Bicocca, (Center B4), School of Medicine and Surgery, University of Milano Bicocca, Milan, Italy

Table 1.

Comparison between no CVAEs and CVAEs group at baseline: main characteristics and haemodynamic ventricular forces

\begin{tabular}{|c|c|c|c|}
\hline Characteristics & $\begin{array}{l}\text { No CVAEs } \\
\mathbf{n}=17\end{array}$ & $\begin{array}{l}\text { CVAEs } \\
\mathrm{n}=\mathbf{2 3}\end{array}$ & $P$ value \\
\hline \multicolumn{4}{|l|}{ General } \\
\hline Age, years old, mean (SD) & $67.9 \pm 8.17$ & $66.3 \pm 7.7$ & 0.533 \\
\hline Male sex, $\mathrm{n}(\%)$ & $6(35.3)$ & $14(60.9)$ & 0.110 \\
\hline \multicolumn{4}{|l|}{ CV risk factors, $\mathrm{n}(\%)$} \\
\hline Tobacco use (prior/current) & $14(35)$ & $9(39.1)$ & 0.878 \\
\hline Arterial Hypertension & $6(35.3)$ & $13(56.5)$ & 0.184 \\
\hline Diabetes & $2(11.8)$ & $2(8.7)$ & 0.749 \\
\hline Chronic renal failure $(\mathrm{eGFR}<60 \mathrm{ml} / \mathrm{m}$ ) & $2(11.8)$ & $2(8.7)$ & 0.749 \\
\hline Ischemic heart disease & $1(5.9)$ & $2(8.7)$ & 0.738 \\
\hline Dyslipidaemia & $1(5.9)$ & $0(0)$ & 0.174 \\
\hline \multicolumn{4}{|l|}{ Office BP values, mean (SD) } \\
\hline $\mathrm{SBP}, \mathrm{mmHg}$ & $120 \pm 17.6$ & $132 \pm 16.7$ & 0.034 \\
\hline $\mathrm{DBP}, \mathrm{mmHg}$ & $68.6 \pm 11.2$ & $77.7 \pm 8.5$ & 0.006 \\
\hline \multicolumn{4}{|l|}{ Echocardiography, mean (SD) } \\
\hline LVMi, g/m2 & $85.6 \pm 15$ & $95.9 \pm 23.2$ & 0.124 \\
\hline LVEF, $\%$ & $63.0 \pm 6.83$ & $61.40 \pm 4.74$ & 0.231 \\
\hline \multicolumn{4}{|l|}{ LV Haemodynamic forces } \\
\hline Longitudinal forces cardiac cycle (LVLF), $\%$ & $12.6 \pm 4.76$ & $9.60 \pm 2.52$ & 0.015 \\
\hline Longitudinal forces systolic (LVsysLF), \% & $16.4 \pm 5.77$ & $12.3 \pm 3.06$ & 0.006 \\
\hline $\begin{array}{l}\text { Longitudinal forces positive systolic acceleration } \\
\text { (LVsys accLV), } \%\end{array}$ & $15.2 \pm 5.21$ & $11.7 \pm 3.26$ & 0.012 \\
\hline Systolic acceleration peak (LVacc_peakLV) & $28.5 \pm 12$ & $19.9 \pm 5.64$ & 0.04 \\
\hline Longitudinal forces systolic ejection (LVejLF), \% & $16.4 \pm 5.8$ & $12.3 \pm 3.7$ & 0.010 \\
\hline Latero-septal forces (LV sysLSF), $\%$ & $2.59 \pm 0.95$ & $1.97 \pm 0.61$ & 0.015 \\
\hline Systolic acceleration work (LVacc workLV) $\mathrm{mJ}$ & $4.58 \pm 1.99$ & $3.00 \pm 1.14$ & 0.003 \\
\hline
\end{tabular}

eGFR = estimated glomerular filtration rate; $\mathrm{LVMi}=$ left ventricular mass indexed to body surface area; $\mathrm{LVEF}=$ left ventricular ejection fraction

Table 2.

Regression analysis and potential cut-off of main haemodynamic forces

\begin{tabular}{|c|c|c|c|c|c|}
\hline Haemodynamic Forces & Cut-off & $\begin{array}{c}\text { No CVAEs } \\
\mathbf{N}=\mathbf{1 7} \\
\mathbf{n}(\%) \\
\end{array}$ & $\begin{array}{c}\text { CVAEs } \\
\text { N=23 } \\
\text { n (\%) } \\
\end{array}$ & OR & P value \\
\hline Longitudinal forces cardiac cycle (LVLF), $\%$ & $<11 \%$ & $6(35.3)$ & $16(69.6)$ & 4.2 & 0.035 \\
\hline Longitudinal forces systolic (LVsysLF), \% & $<15.4 \%$ & $7(41.2)$ & $20(86.9)$ & 9.5 & 0.004 \\
\hline $\begin{array}{l}\text { Longitudinal forces positive systolic acceleration } \\
\text { (LVsys_accLV), \% }\end{array}$ & $<12.7 \%$ & $5(29.4)$ & $15(65.2)$ & 4.5 & 0.029 \\
\hline Systolic acceleration peak (LVacc_peakLV) & $<27$ & $9(52.9)$ & $21(91.3)$ & 9.3 & 0.012 \\
\hline Longitudinal forces systolic ejection ( $\overrightarrow{L V e j L F}), \%$ & $<13.5 \%$ & $5(29.4)$ & $16(69.6)$ & 5.5 & 0.015 \\
\hline Latero-septal forces (LV_sysLSF), \% & $<2.4 \%$ & $7(41.2)$ & $19(82.6)$ & 6.8 & 0.010 \\
\hline Systolic acceleration work (LVacc_workLV), $\mathrm{mJ}$ & $<3.9 \mathrm{~mJ}$ & $5(29.4)$ & $18(78.3)$ & 8.6 & 0.003 \\
\hline
\end{tabular}


Introduction: Heterogeneous results have been obtained regarding the presence of Target Organ Damage (TOD) in subjects with highnormal Blood Pressure (BP) value (systolic 130-139, diastolic 85-89 $\mathrm{mmHg}$ ).

Aim: Aim of our study was to assess the prevalence of TOD in healthy subjects with high-normal BP comparing them with subjects with $\mathrm{BP}$ values that are considered normal $(<130 / 85 \mathrm{mmHg})$ or indicative of hypertension ( $\geq 140 / 90 \mathrm{mmHg}$ ).

Methods: 755 otherwise healthy subjects participated at the present analysis. TOD was evaluated as Pulse Wave Velocity (PWV), Left Ventricular Mass Index (LVMI) and carotid Intima-Media Thickness (IMT) and plaque.

Results: When subjects were classified according to BP levels we found that high-normal BP group showed intermediate values of PWV and LMVI with higher value of IMT. This corresponds to intermediate prevalence of arterial stiffness (PWV $>10 \mathrm{~m} / \mathrm{s}: 3.4 \%$ vs $2.3 \%$ for normal and $9.6 \%$ for high BP groups, $\mathrm{p}=0.0014$ ), Left Ventricular Hypertrophy-LVH (32.4\% vs $25.7 \%$ for normal and $46.6 \%$ for high BP groups, $\mathrm{p}=0.0001$ ) while there were no differences for IMT $>0.9$ or carotid plaque. At multivariable analysis the odds of having a PWV $>10 \mathrm{~m} / \mathrm{s}(\mathrm{OR}=1.75,95 \%$ C.I. 0.59-5.16), an IMT $>0.9 \mathrm{~mm}(\mathrm{OR}=1.81,95 \%$ C.I. $0.60-5.00)$ or a $\mathrm{LVH}(\mathrm{OR}=1.1$, $95 \%$ C.I. 0.72-1.67) in the high-normal group resulted not different to the normal group.

Conclusions: In our otherwise healthy population, high-normal BP values were not related to aortic, carotid or cardiac TOD.

\section{HAEMODYNAMIC CHARACTERISTICS AND EVOLUTION DURING FOLLOW-UP OF DIFFERENT HYPERTENSIVE SUBTYPES IN YOUNG STAGE I HYPERTENSIVES}

Francesca Saladini ${ }^{1-2}$, Lucio Mos ${ }^{3}$, Olga Vriz ${ }^{3}$, Andrea Mazzer ${ }^{4}$, Claudio Fania ${ }^{1}$, Marcello Rattazzi ${ }^{5}$, Palatini Palatini ${ }^{1}$

${ }^{1}$ Department of Medicine, University of Padua, Padua, Italy, ${ }^{2}$ Cardiology Unit, Cittadella Town Hospital, ALSS 6 Euganea, Italy, ${ }^{3}$ Cardiology Unit, San Daniele del Friuli Town Hospital, San Daniele del Friuli, Italy, ${ }^{4}$ Medicine Unit, Vittorio Veneto Town Hospital, Vittorio Veneto, Italy, ${ }^{5}$ Medicine Unit, Ca' Foncello Hospital, Treviso, Italy

Introduction: Diverse hypertensive subtypes present different evolution in young subjects. In particular, blood pressure (BP) evolution of young isolated systolic hypertensives (ISH) is still debated.

Aim: We evaluated haemodynamic characteristics and BP evolution of different hypertensive subtypes, untreated at the baseline, during 7 years of follow-up.

Methods: 1026 subjects from the HARVEST study, were divided according to their enrolment BP levels into: 81 normotensives (NT), 146 ISH, 281 isolated diastolic hypertensives (IDH) and 698 systolicdiastolic hypertensives (SDH).

Results: Males were more frequent in each hypertensive subgroup and in particular among ISH (90.4\%). ISH were younger (25.6 \pm 6.6 years, $\mathrm{p}<0.001)$ and thinner compared to SDH $(24.6 \pm .2 .6$ vs 25.8 $\pm 3.6 \mathrm{~kg} / \mathrm{m} 2, \mathrm{p}=0.025)$, were more active in sports $(55.5 \%, \mathrm{p}<$ $0.001)$, drank less alcohol and coffee compared to the other groups. ISH presented also an increased white coat effect both for systolic BP $(17.6 \pm 12.4 \mathrm{mmHg})$ and heart rate $(\mathrm{HR})(6.0 \pm 9.3 \mathrm{bpm}, \mathrm{p}<0.001)$, compared to others. With regard to haemodynamic characteristics ISH were characterized by an increased cardiac output $(82.8 \pm .15 .3$ $\mathrm{ml}, \mathrm{p}=0.009)$ and reduced peripheral resistances $(1371.3 \pm 281.5$ Dyne/sec/ $\left.\mathrm{cm}^{-5}, \mathrm{p}<0.001\right)$, compared to the other hypertensive subtypes. During follow-up systolic BP significantly decreased (-7.6 $\pm 14.4 \mathrm{mmHg})$ among ISH compared to the others $(\mathrm{p}<0.001$ for all comparisons), while diastolic BP slightly increased $(3.7 \pm 9.1 \mathrm{mmHg}$; $\mathrm{p}<0.001$ vs IDH e SDH). HR decreased in all categories, but to a higher extent among ISH $(-3.4 \pm 10.8 \mathrm{bpm}, \mathrm{p}=0.002)$. The percentage of patients who started pharmacological treatment was lower among ISH (41.1\%) compared to the other hypertensive subgroups ( $\mathrm{p}$ $<0.001)$.

Conclusions: Baseline characteristics and BP evolution during follow-up seemed to be favourable among ISH compared to the other hypertensive subtypes. However, more studies with longer follow-up and harder hand point are needed to establish whether the prognosis of these young patients is really benign.

\section{DIFFERENCES IN DIAGNOSIS AND TREATMENT OF HYPERTENSIVE URGENCY AND EMERGENCY ACCORDING TO THE DIFFERENT SPECIALITY OF THE PHYSICIANS WHO DEAL WITH ACUTE INCREASE OF BLOOD PRESSURE (DATA FROM GEAR STUDY)}

Francesca Saladini ${ }^{1,2}$, Costantino Mancusi ${ }^{3}$, Fabio Bertacchini ${ }^{4}$, Francesco Spannella $^{5 a-b}$, Alessandro Maloberti ${ }^{6 a-b}$, Alessandra Giavarini $^{7}$, Martina Rosticci ${ }^{8}$, Rosa Maria Bruno9', Giacomo Pucci ${ }^{10}$, Davide Grassi ${ }^{11}$, Martino Pengo ${ }^{12}$, Maria Lorenza Muiesan ${ }^{4}$

${ }^{I}$ Department of Medicine, University of Padua, Padua, Italy, ${ }^{2}$ Cardiology Unit, Cittadella Town Hospital, Padua, Italy, ${ }^{3}$ Hypertension Research Center, Department of Advanced Biomedical Science, Federico II University of Naples, Italy, ${ }^{4}$ Internal Medicine, University of Brescia-ASST Spedali Civili di Brescia, Brescia, Italy, ${ }^{5 a}$ Internal Medicine and Geriatrics, IRCCS-INRCA, Ancona, ${ }^{b}$ Department of Clinical and Molecular Sciences, University "Politecnica delle Marche”, Ancona, Italy, ${ }^{6 a}$ Cardiology 4, ASST Niguarda Hospital, Milan, ${ }^{b}$ School of Medicine and Surgery, MilanoBicocca University, Milan, Italy, ${ }^{7}$ Cardiology Unit, Luigi Sacco Hospital, ASST Fatebenefratelli Sacco, Milan, Italy, ${ }^{8}$ Medicine and Surgery Sciences Department, Alma Mater Studiorum University of Bologna, Bologna, Italy, ${ }^{9}$ Department of Clinical and Experimental Medicine, University of Pisa. Pisa, Italy, ${ }^{10}$ Internal Medicine, Department of Medicine, University of Perugia, Terni University Hospital, Terni, Italy, ${ }^{11}$ Department of Internal medicine and Public Health, University of Aquila, L'Aquila, Italy, ${ }^{12}$ Department of Cardiovascular, Neural and Metabolic Sciences, IRCCS Istituto Auxologico Italiano, Milan, Italy

Introduction: Diagnosis and treatment of hypertension emergency (HE) and urgency (HU) may vary according to the physicians involved and the hospital setting of the treatment.

Aim: The aim of this study was to investigate differences for management of HE and HU according to different doctors' specialities. Methods: The young investigators of the Italian Society of Hypertension developed a 23 -item questionnaire spread by e-mail invitation to the members of Italian Scientific societies involved in the field of Emergency Medicine and Hypertension.

Results: 665 questionnaires have been collected: $59.7 \%$ from Emergency Department (ED), 22\% from Emergency and Urgency Medicine wards, $8.7 \%$ from Cardiology Unit, $5.7 \%$ form Internal Medicine and $3.9 \%$ form Intensive Care Unit or Stroke Unit. ED physicians are more familiar with lung ultrasound $(43.8 \%$ vs $32.1 \% \mathrm{p}$ $=0.005)$, administration of intravenous urapidil $(67.8 \%$ vs $54.9 \% \mathrm{p}=$ $0.003)$ and oral captopril (59.6\% vs $39.2 \% \mathrm{p}=0.002)$, compared to 
other specialties. Cardiologists more frequently define epistaxis (55.2\% vs $33.2 \%, \mathrm{p}=0.001)$, tinnitus $(50.0 \%$ vs $35.0 \%, \mathrm{p}=0.027)$ and conjunctival haemorrhages (70.7 vs $38.5, \mathrm{p}<0.0001)$ as possible presentation of HE compared to ED physicians. Moreover, they report the highest prescription of i.v. furosemide $(79.3 \%$ vs $54.4 \% \mathrm{p}=$ $0.001)$ and s.1. nifedipine ( $43.1 \%$ vs $22.7 \%, \mathrm{p}=0.004)$. The approach of physicians working in Emergency and Urgency Medicine (22\%) and ED is very similar in terms of suggestive signs and symptoms, proposed exams, drugs administrated in front of HE and HU. The former group more frequently suggest specialistic visits $(25.9 \%$ vs $13.7 \%, \mathrm{p}=0.007)$, or ambulatory blood pressure monitoring $(30.4 \%$ vs $21.6 \%, \mathrm{p}=0.030$ ) compared to the latter one as follow-up after discharge form ED.

Conclusions: Treatment of HE and HU may be different according to the speciality of the doctor who faces these problems. Educational initiatives should be done to standardize treatment protocols and to improve medical knowledge on this topic.

\section{HIGH HEART RATE AMPLIFIES THE RISK OF CARDIOVASCULAR MORTALITY ASSOCIATED WITH HYPERURICEMIA}

Paolo Palatini, Gianfranco Parati, Agostino Virdis, Gianpaolo Reboldi, Stefano Masi, Alessandro Mengozzi, Edoardo Casiglia, Valerie Tikhonoff, Arrigo F.G. Cicero, Andrea Ungar, Giulia Rivasi, Massimo Salvetti, Carlo M. Barbagallo, Michele Bombelli, Raffaella Dell'Oro, Berardino Bruno, Luciano Lippa, Lanfranco D'Elia, Paolo Verdecchia, Fabio Angeli, Francesca Mallamaci, Massimo Cirillo, Marcello Rattazzi, Pietro Cirillo, Loreto Gesualdo, Alberto Mazza, Cristina Giannattasio, Alessandro Maloberti, Massimo Volpe, Giuliano Tocci, Georgios Georgiopoulos, Guido Iaccarino, Pietro Nazzaro, Ferruccio Galletti, Claudio Ferri, Giovambattista Desideri, Francesca Viazzi, Roberto Pontremoli, Maria Lorenza Muiesan, Guido Grassi, Claudio Borghi

Working Group on Uric Acid and Cardiovascular Risk of the Italian Society of Hypertension

Introduction: Whether the association between uric acid (UA) and cardiovascular disease is influenced by some facilitating factors is unclear.

Aim: To investigate whether the risk of cardiovascular mortality (CVM) associated with elevated UA was modulated by the level of resting heart rate.

Methods: The HURRAH database was constructed by merging data from several cohorts recruited within the Italian Centres of Hypertension. Multivariable Cox analyses were made in 19.128 participants in whom anthropometric measures, UA, heart rate, office systolic and diastolic blood pressures (BP), fasting blood lipid and glycaemic profiles, estimated glomerular filtration rate (eGFR), and information on cardiovascular risk factors were available. To assess the incremental predictive value for CVM risk afforded by risk factors, we calculated the continuous net reclassification improvement (cNRI), and the integrated discrimination improvement (IDI).

Results: Mean age \pm SD was $53.3 \pm 14.6$ years, office systolic BP was $143.0 \pm 23.6 \mathrm{~mm} \mathrm{Hg}$, and diastolic BP was $84.7 \pm 12.5 \mathrm{~mm} \mathrm{Hg}$. UA was higher in men $(5.19 \pm 1.40 \mathrm{mg} / \mathrm{dL})$ than in women $(4.85 \pm$ $1.41 \mathrm{mg} / \mathrm{dL})(\mathrm{p}<0.0001)$, whereas heart rate was lower in the former $(70.8 \pm 12.5$ versus $72.9 \pm 12.0 \mathrm{bpm}, \mathrm{p}<0.0001)$. During a median follow-up of 11.2 years there were 1381 cases of CVM. In multivariable Cox models both UA and heart rate either considered as continuous or categorical variables were independent predictors of CVM. However, the risk of CVM related to high UA $(\geq 5.5 \mathrm{mg} / \mathrm{dL}$, top tertile) was much lower in the subjects with heart rate $<$ median [
(71.3 bpm, adjusted hazard ratio 1.38 (95\%CI, 1.20-1.59)] than in those with heart rate $\geq$ median [2.09 (95\% CI, 1.75-2.51)]. In the participants stratified by heart rate tertile, the risk related to hyperuricemia was 2.38 (95\% CI, 1.82-3.10) in people with heart rate $\geq 76$ bpm and was 1.24 (95\% CI, 0.92-1.67) in those with heart rate $<66$ bpm. A statistically significant cNRI was obtained after adding either UA or heart rate to a multivariable model including age, sex, smoking, diabetes, total cholesterol, eGFR, and systolic BP. Separation between events and non-events, after the addition of UA or heart rate, resulted in a significant IDI. Significant cNRI and IDI were obtained also when UA was included in a risk predictive model including heart rate. Similar to what was demonstrated in the overall population, when stratifying the non diabetic population according to UA and heart rate categories, the $\mathrm{CV}$ mortality risk was increased in the middle and top UA tertiles only in the setting of higher heart rate. Further sensitivity analyses showed that similar results were obtained in the hypertensive patients, in the participants 65 year of age or older and in the subjects not taking beta-blockers

Conclusions: The present data suggest that the contribution of UA to determining cardiovascular mortality is modulated by the level of heart rate supporting the hypothesis that activation of the sympathetic nervous system facilitates the action of UA as a cardiovascular risk factor.

\section{THE ASSESSMENT OF THE GLOBAL CARDIOVASCULAR RISK IN A HYPERTENSION CENTER: A PROSPECTIVE MANAGEMENT MODEL OF RISK AND POSSIBLE REPERCUSSIONS ON THE REGIONAL HEALTH SYSTEM}

Alberto Mazza ${ }^{1}$, Giorgio Lorenzo Colombo ${ }^{2}$, Sergio Di Matteo ${ }^{2}$, Giacomo Matteo Bruno ${ }^{2}$, Alice Bresolin ${ }^{3}$, Laura Schiavon ${ }^{1,4}$, Gioia Torin $^{1,4}$, Ciro Rossetti ${ }^{1,4}$, Antonella Paola Sacco ${ }^{1,4}$, Marco Marzolo ${ }^{5}$, Ezio Di Giacomo ${ }^{1,5}$, Rossana Redi ${ }^{1}$, Lorella Boreggio ${ }^{1}$, Marina Bellato $^{1}$, Stefania Cappello ${ }^{1,6}$, Barbara Tamiello ${ }^{1,6}$, Alessandro Camerotto $^{7}$, Stefano Cuppini ${ }^{4}$

${ }^{1}$ Unit of Hypertension and ESH Excellence Hypertension Centre, Department of Internal medicine, Santa Maria della Misericordia General Hospital, Rovigo, Italy, ${ }^{2}$ CEFAT-Center of Pharmaceuticals Economics and Medical Technologies Evaluation, Drug Science Department, Pavia University, Pavia, Italy, ${ }^{3}$ School of Internal Medicine, University of Padua, Padua, Italy, ${ }^{4}$ Internal Medicine Unit, Department of Internal Medicine, Santa Maria della Misericordia General Hospital, Rovigo, Italy, ${ }^{5}$ Unit of Angiology, Internal Medicine Unit, Santa Maria della Misericordia General Hospital, Rovigo, Italy, ${ }^{6}$ Outpatient office and Day-Hospital serviceDepartment of Internal Medicine, Santa Maria della Misericordia General Hospital, Rovigo, Italy, ${ }^{7}$ Unit of Laboratory Medicine, Santa Maria della Misericordia General Hospital, Rovigo, Italy

Introduction: In the hypertensive subjects, national and international guidelines recommend cardiovascular $(\mathrm{CV})$ risk assessment using the Systematic-Coronary-Risk-Estimation (SCORE) and the adoption of specific measures aimed at controlling blood pressure levels and risk factors associated with hypertension (HT), which has known, they have a strong impact on the prevention of $\mathrm{CV}$ diseases and the resources of health systems.

Aim: To evaluate the effectiveness of an HT management model to modulate prospectively the overall CV risk associated with HT.

Methods: The study participants are part of a population of 2.120 subjects consecutively recruited in the Hypertension centre from 
January 2005 to April 2021. To stratify the CV risk with the SCORE algorithm, at baseline 889 subjects with a history of cardio-cerebrovascular events, diabetes or chronic kidney disease and 40 subjects at follow-up, for whom the SCORE was no longer applicable, were excluded. Therefore, 1.191 subjects (51\% males) aged 40-70 years (mean $54.2 \pm 8.3$ years) were analyzed and in them, the SCORE at baseline and follow-up (mean $4.7 \pm 3.8$ years) was re-calculated if sub-clinical organ damage (OD) was discovered. This latter was defined as left ventricular hypertrophy (i.e. left ventricular mass index $>115 \mathrm{~g} / \mathrm{m}^{2}$ in males and $>95 \mathrm{~g} / \mathrm{m}^{2}$ in females), carotid plaque $(>50 \%)$ at the eco-color doppler ultrasonography or albuminuria $(>30$ $\mathrm{mg} / 24 \mathrm{~h})$. The OD identified patients in the SCORE class between $5-10 \%$. For the entire follow-up, non-pharmacological hypertension treatment was carried out, lifestyle modification and smoking abolition were recommended also with the support of educational material and, when indicated, anti-hypertensive and lipid-lowering therapy was started. The change in SCORE risk at follow-up was analyzed with Pearson's $\mathrm{X}^{2}$ test.

Results: At baseline, $16.4 \%$ of subjects had SCORE $<1 \%$, the $63.2 \%$ had $1-5 \%$, the $17.0 \%$ had $5-10 \%$ and the $3.4 \%$ had $>10 \%$, respectively. The OD was found in $34.9 \%$ of patients with SCORE $<$ $1 \%$, in the $57.6 \%$ with $1-5 \%$, in the $74.1 \%$ with $5-10 \%$ and in the $72 \%$ with SCORE $>10 \%$, respectively. The presence of OD reclassified the SCORE as follows: $-35 \%$ for $<1 \%,-57.2 \%$ for $1-5 \%,+255 \%$ for $5-10 \%$ while for $>10 \%$ it remained unchanged. Comparing the SCORE at follow-up versus baseline (Figure) there was a significant redistribution of the global $\mathrm{CV}$ risk of patients with a reduction of the high and very-high $\mathrm{CV}$ risk $(-46 \%$ and $-77 \%$ respectively, $\mathrm{p}<$ 0.0001).

Conclusions: A CV risk management model in primary prevention of $\mathrm{CV}$ diseases is proposed, for which cost-effectiveness analyzes are ongoing.

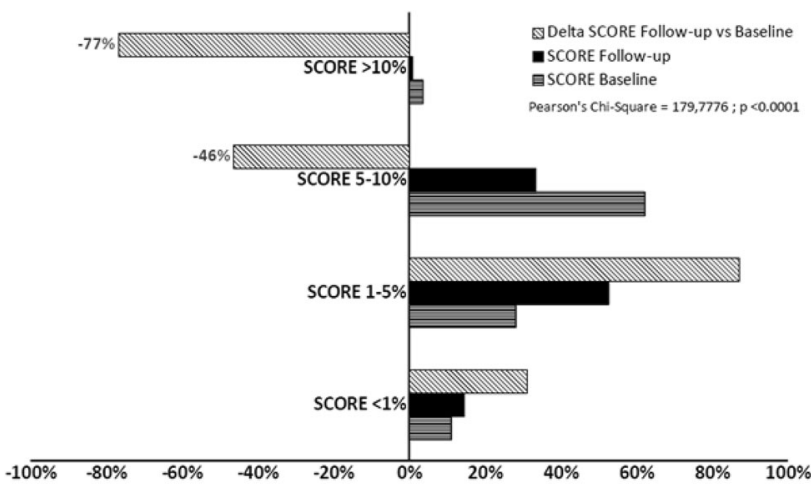

Systematic-Coronary-Risk-Estimation (SCORE) change

\section{WHITE-COAT HYPERTENSION WITHOUT ORGAN DAMAGE: IMPACT ON LONG-TERM CARDIOVASCULAR AND ALL CAUSE MORTALITY}

Gino Seravalle ${ }^{1}$, Rita Facchetti ${ }^{1}$, Fosca Quarti Trevano ${ }^{1}$, Cesare Cuspidi1, Giuseppe Mancia ${ }^{2}$, Guido Grassi ${ }^{1}$

${ }^{1}$ Clinica Medica, University of Milano Bicocca, Milan, Italy, ${ }^{2}$ Policlinico of Monza and University of Milano Bicocca, Milan, Italy

Introduction: According to some guidelines white-coat hypertension $(\mathrm{WCH})$ carries no increased cardiovascular $(\mathrm{CV})$ risk in absence of organ damage (OD) but no data are available on this issue.
Aim: We aimed at determining the $\mathrm{CV}$ risk of $\mathrm{WCH}$ without $\mathrm{OD}$ compared to no-OD normotensives and sustained hypertensives Methods: We evaluated the $\mathrm{CV}$ and total mortality of WCH with noOD enrolled in the Pressioni Arteriose Monitorate E Loro Associazioni (PAMELA) study over a median 17.7 years' follow-up. Data were compared with those from no-OD in- and out-of-office normotensives and hypertensives based on office, home and ambulatory blood pressure. Analysis was extended to normotensives, $\mathrm{WCH}$ and hypertensive individuals with OD

Results: During the follow-up there were 68 and 212 fatal CV and all cause events, respectively. Compared with individuals with OD, noOD individuals displayed a much lower incidence of events. Following adjustment for confounders, no-OD WCH exhibited a risk of fatal CV events lower than that of hypertensive patients but greater of normotensives $(\mathrm{P}=0.04)$. Compared with no-OD normotensives, noOD WCH individuals also exhibited a significantly greater 10 year adjusted risk to develop left ventricular hypertrophy or renal damage $(\mathrm{OR}=2.0 ; 95 \% \mathrm{CI}: 1.3-2.9 ; \mathrm{P}=0.001)$ as well as to progress to outof-office hypertension $(\mathrm{OR}=2.53 ; 95 \% \mathrm{CI}: 1-5-4.4 ; \mathrm{P}=0.001)$

Conclusions: In absence of OD WCH is not a clinically innocent condition, the reason being not only its independent association with a greater long-term mortality, but also its greater propensity to progress towards clinical states of documented greater $\mathrm{CV}$ risk and poorer outcome.

\section{ASSESSING FRAILTY IN HYPERTENSIVE OLDER ADULTS: A COMPARISON OF DIFFERENT FRAILTY MEASURES}

Giulia Rivasi, Giada Turrin, Virginia Tortù, Maria Flora D'Andria, Antonio Coscarelli, Ludovica Ceolin, Martina Rafanelli, Enrico Mossello, Andrea Ungar

Hypertension Clinic, Division of Geriatric and Geriatric Intensive Care Medicine, Careggi Hospital and University of Florence, Florence, Italy.

Introduction: Frailty has been poorly investigated in hypertensive older adults to date and the most appropriate tool to quantify frailty in this population has yet to be identified.

Aim: The present study assessed frailty prevalence in a sample of hypertensive older adults according to the most widely used frailty measure tools, aiming at analysing their agreement and the corresponding frailty phenotype.

Methods: Hypertensive patients aged 75 or older referred to the Hypertension Clinic and the Memory Clinic of the Geriatric Department of Careggi Hospital, Florence, were enrolled. The following frailty and physical performance measure tools were applied: Fried Frailty Phenotype (FFP), Frailty Index, Clinical Frailty Scale (CFS), Frailty Postal Score (FPS), Short Physical Performance Battery (SPPB), gait velocity. Cohen's kappa coefficient was calculated to assess the agreement between measures. Multiple logistic regression was used to identify variables that best predicted frailty according to different measures after adjustment for physical performance, disability, comorbidities, depressive symptoms and family status.

Results: Ninety-one patients were enrolled (mean age $81,59 \%$ female). Frailty prevalence varied according to frailty measure, ranging from $31 \%$ (gait velocity) to $50 \%$ (CFS). The Cohen's kappa coefficient indicated moderate agreement of the FFP with Frailty Index, gait velocity and SPPB, and of Frailty Index with CFS and SPPB $(K=0.6-0.8)$. Agreement was poor among the remaining measures $(K<0.6$, Figure 1$)$. Multivariate analysis revealed the following independent associations: FFP with reduced gait velocity, 
Frailty Index and CFS with disability in Instrumental Activities of Daily Life (IADL) and Charlson Comorbidity Index, physical performance measures with disability in IADL. Depressive symptoms were significantly associated with frailty according to all the frailty measures used.
Conclusions: Frailty is common in older adults with hypertension, although prevalence varies depending on the measure used. Available measure tools may capture different subtypes of frailty.

Table 1. Agreement among frailty measures according to Cohen's kappa coefficient.

\begin{tabular}{|c|c|c|c|c|c|c|}
\hline & $\begin{array}{l}\text { Fried Frailty } \\
\text { Phenotype }\end{array}$ & Frailty Index & $\begin{array}{l}\text { Clinical Frailty } \\
\text { Scale }\end{array}$ & $\begin{array}{l}\text { Frailty Postal } \\
\text { Score }\end{array}$ & $\begin{array}{c}\text { Gait velocity } \\
<0.8 \mathrm{~m} / \mathrm{sec}\end{array}$ & $\begin{array}{c}\text { SPPB score } \\
\leq 8\end{array}$ \\
\hline $\begin{array}{l}\text { Fried Frailty } \\
\text { Phenotype } \\
\text { (Frail } n=37)\end{array}$ & - & $\begin{array}{c}0.633 \\
(p<0.001)\end{array}$ & $\begin{array}{c}0.515 \\
(p<0.001)\end{array}$ & $\begin{array}{c}0.583 \\
(p<0.001)\end{array}$ & $\begin{array}{c}0.692 \\
(p<0.001)\end{array}$ & $\begin{array}{c}0.660 \\
(p<0.001)\end{array}$ \\
\hline $\begin{array}{c}\text { Frailty Index } \\
\text { (Frail } n=35 \text { ) }\end{array}$ & $\begin{array}{c}\mathrm{N}=28 \\
\text { (Expected 14) }\end{array}$ & - & $\begin{array}{c}0.692 \\
(p<0.001)\end{array}$ & $\begin{array}{c}0.578 \\
(p<0.001)\end{array}$ & $\begin{array}{c}0.523 \\
(p=0.001)\end{array}$ & $\begin{array}{c}0.749 \\
(p<0.001)\end{array}$ \\
\hline $\begin{array}{c}\text { Clinical Frailty } \\
\text { Scale } \\
\text { (Frail } n=28)\end{array}$ & $\begin{array}{c}\mathrm{N}=30 \\
\text { (Expected 18) }\end{array}$ & $\begin{array}{c}\mathrm{N}=33 \\
\text { (Expected 17) }\end{array}$ & - & $\begin{array}{c}0.339 \\
(p=0.002)\end{array}$ & $\begin{array}{c}0.511 \\
(p=0.001)\end{array}$ & $\begin{array}{c}0.538 \\
(p<0.001)\end{array}$ \\
\hline $\begin{array}{c}\text { Frailty Postal } \\
\text { Score } \\
\text { (Frail } n=27)\end{array}$ & $\begin{array}{c}\mathrm{N}=26 \\
\text { (Expected 13) }\end{array}$ & $\begin{array}{c}\mathrm{N}=25 \\
\text { (Expected 13) }\end{array}$ & $\begin{array}{c}\mathrm{N}=24 \\
(\text { Expected 16) }\end{array}$ & - & $\begin{array}{c}0.418 \\
(p=0.009)\end{array}$ & $\begin{array}{c}0.517 \\
(p<0.001)\end{array}$ \\
\hline $\begin{array}{r}\text { Gait velocity } \\
\text { (Frail } n=27 \text { ) }\end{array}$ & $\begin{array}{c}\mathrm{N}=27 \\
\text { (Expected 12) }\end{array}$ & $\begin{array}{c}\mathrm{N}=23 \\
\text { (Expected 12) }\end{array}$ & $\begin{array}{c}\mathrm{N}=27 \\
\text { (Expected 16) }\end{array}$ & $\begin{array}{c}\mathrm{N}=20 \\
\text { (Expected 11) }\end{array}$ & - & $\begin{array}{c}0.577 \\
(p<0.001)\end{array}$ \\
\hline $\begin{array}{c}\text { SPPB } \\
(\text { Frail } n=38)\end{array}$ & $\begin{array}{c}\mathrm{N}=30 \\
\text { (Expected 16) }\end{array}$ & $\begin{array}{c}\mathrm{N}=31 \\
\text { (Expected 15) }\end{array}$ & $\begin{array}{c}\mathrm{N}=31 \\
\text { (Expected 19) }\end{array}$ & $\begin{array}{c}\mathrm{N}=25 \\
\text { (Expected 14) }\end{array}$ & $\begin{array}{c}\mathrm{N}=25 \\
\text { (Expected 13) }\end{array}$ & - \\
\hline
\end{tabular}

SPPB, Short Physical Performance Battery

Table 2. Multivariate logistic regression to identify variables associated with frailty according to different measures (OR, 95\% confidence intervals)

\begin{tabular}{|l|c|c|c|c|c|c|}
\hline & $\begin{array}{c}\text { Disability in } \\
\text { IADL }\end{array}$ & $\begin{array}{c}\text { Gait velocity <0.8 } \\
\mathbf{m} / \mathbf{s e c}\end{array}$ & $\begin{array}{c}\text { Dementia / } \\
\text { Mild } \\
\text { Cognitive } \\
\text { Impairment }\end{array}$ & $\begin{array}{c}\text { Depressive } \\
\text { symptoms }\end{array}$ & $\begin{array}{c}\text { Living alone } \\
\text { Charlson } \\
\text { Comorbidity } \\
\text { Index }\end{array}$ \\
\hline Fried & 1.216 & $\mathbf{2 6 . 7 5 2}$ & 1.181 & $\mathbf{6 . 2 9 5}$ & 1.902 & 0.945 \\
$(0.213-6.958)$ & $(4.595-155.749)$ & $(0.206-6.789)$ & $(1.410-28.114)$ & $(0.392-9.232)$ & $(0.552-1.620)$ \\
\hline Frailty & $\mathbf{2 2 . 3 9 7}$ & 1.279 & 1.409 & $\mathbf{2 1 . 5 7 7}$ & 1.273 & $\mathbf{2 . 0 5 3}$ \\
Index & $(3.006-166.850)$ & $(0.283-5.788)$ & $(0.257-7.716)$ & $(3.177-146.554)$ & $(0.268-6.042)$ & $(1.095-3.848)$ \\
\hline CFS & $\mathbf{1 9 . 1 8 8}$ & 2.127 & 1.319 & $\mathbf{1 4 . 0 5 2}$ & 1.623 & 1.894 \\
& $(3.455-106.572)$ & $(0.408-11.071)$ & $(0.242-7.195)$ & $(2.243-88.030)$ & $(0.384-6.854)$ & $(1.043-3.440)$ \\
\hline FPS & 1.284 & 4.072 & 0.982 & $\mathbf{4 . 6 7 2}$ & 2.343 & 1.120 \\
& $(0.311-5.304)$ & $(0.980-16.910)$ & $(0.245-3.942)$ & $(1.400-15.592)$ & $(0.647-8.482)$ & $(0.748-1.676)$ \\
\hline SPPB & $\mathbf{4 . 0 7 3}$ & - & 1.485 & $\mathbf{6 . 0 1 5}$ & 0.923 & 1.327 \\
& $(1.164-14.250)$ & & $(0.403-5.466)$ & $(1.829-19.780)$ & $(0.258-3.300)$ & $(0.881-1.998)$ \\
\hline Gait & $\mathbf{9 . 6 5 6}$ & - & 2.911 & $\mathbf{1 2 . 3 5 1}$ & 0.720 & 1.129 \\
velocity & $(1.861-50.110)$ & & $(0.607-3.970)$ & $(2.637-57.853)$ & $(0.139-3.745)$ & $(0.691-1.847)$ \\
\hline
\end{tabular}

Variables included in the model: age, sex, disability in Instrumental Activities of Daily Living (IADL), gait velocity $<0.8 \mathrm{~m} / \mathrm{s}$, dementia/mild cognitive impairment, depressive symptoms according to the Geriatric Depression Scale, living alone, Charlson Comorbidity Index (not age-adjusted).

IADL, Instrumental Activities of Daily Life; CFS, Clinical Frailty scale; FPS, Frailty Postal Score; SPPB, Short Physical Performance Battery 


\section{INEQUALITIES IN ENROLLMENT OF WOMEN AND RACIAL MINORITIES IN TRIALS TESTING URIC ACID LOWERING DRUGS}

\author{
Arrigo F. G. Cicero ${ }^{1,2}$, Federica Fogacci ${ }^{1,2}$, Antonio Di Micoli ${ }^{2}$, \\ Daniela Degli Esposti ${ }^{2}$, Claudio Borghi ${ }^{1,2}$
}

${ }^{1}$ Hypertension and Cardiovascular Risk Factors Research Group, Medical and Surgical Sciences Department, University of Bologna, Italy, ${ }^{2}$ IRCCS-University Hospital of Bologna, Bologna, Italy

Introduction: Adequate enrollment of men and women in pharmacological research is crucial to discern any existing sex-related difference in drugs' effects and safety.

Aim: We investigated sex inequalities in clinical trials testing serum uric acid (SUA) lowering drugs and analyzed the temporal trends of participation among the pre-specified demographic group.

Methods: Data were collected from publications of clinical trials testing SUA-lowering drugs. Linear regression analysis was performed to assess the relation between studies' publication year and proportion of women enrolled in clinical studies.

Results: A total of 627 patients were women. The participation of women as an average of the percentage of women enrolled in each individual study significantly decreased over the time $(r=-0.43$, P-value $=0.02$ ).

There was a statistically significant difference in enrollment of women among trials testing different SUA-lowering drugs, with the highest representation in rasburicase $(71.1 \%)$ and the lowest representation of women in dotinurad $(0.8 \%)$.

Women were proportionally underrepresented compared with their share of the population with asymptomatic hyperuricemia, overall (participation-to-prevalence ratio $(\mathrm{PPR})=0.34)$, in trials testing xanthine oxiase inhibitors $(P P R=0.38)$ and uricosurics $(P P R=0.29)$, and in trials with febuxostat, allopurinol, pegloticase, halofenate/ arhalofenate, verinurad, lesinurad and dotinurad. Women were proportionally underreppresented also compared with their share of the population with gout, overall $(\mathrm{PPR}=0.69)$ and in trials testing XOIs (PPR $=0.69)$, uricosurics $(\mathrm{PPR}=0.68)$, and all SUA-lowering drugs excepted for rasburicase, pegloticase and topiroxostat.

Conclusions: Our analysis shows that women are underrepresented in controlled clinical trials testing SUA-lowering drugs, with similar pattern across drug classes.

\section{THE SIIA ONLINE ARTERIAL HYPERTENSION QUESTIONNAIRE}

Guido Iaccarino ${ }^{1}$, Massimo Salvetti ${ }^{2}$, Leonardo Sechi ${ }^{3}$, Claudio Borghi $^{4}$, Massimo Volpe ${ }^{5}$, Maria Lorenza Muiesan ${ }^{2}$, Claudio Ferri ${ }^{6}$, Guido Grassi ${ }^{7}$

${ }^{1}$ CIRIAPA, Federico II University, Naples, Italy, ${ }^{2}$ Dipartimento di Scienze Cliniche e Sperimentali University of Brescia, Brescia, Italy, ${ }^{3}$ Dipartimento di Area Medica, University of Udine, Udine, Italy, ${ }^{4}$ Dipartimento di Scienze Mediche e Chirurgiche University of Bologna, Bologna, Italy, ${ }^{5}$ Dipartimento di Medicina Clinica e Molecolare University of Roma La Sapienza, Rome, Italy, ${ }^{6}$ Dipartimento di Medicina Clinica, Sanità Pubblica, Scienze della Vita e dell'Ambiente, University of L'Aquila, L'Aquila, Italy,

${ }^{7}$ Dipartimento di Medicina e Chirurgia, University of Milan, Bicocca, Milan, Italy

Introduction: Hypertension awareness among the general population is increasing, and the fast evolution of the internet offers the possibility to monitor the people perception of the condition.
Aim: We analyzed the responses collected in the period from 1 May to 26 May 2021.

Methods: On the occasion of World Hypertension Day, a publicly accessible questionnaire was placed on the SIIA website to be filled out anonymously, available online all year round ( https://bit.ly/31kUzSZ).

Results: Respondents had an average age of $58.1 \pm 18.1$ years; $78 \%$ are male, and the BMI $26.3 \pm 6.0$. They are geographically distributed in the regions of northern and central Italy. The majority of respondents stated that they were hypertensive $(88 \%)$ while $12 \%$ cumulatively declared that they were not $(6 \%)$ or did not know they were hypertensive $(6 \%)$. Family history for hypertension was reported in $54.6 \%$ of cases, while that for diabetes in $30 \%$. $18 \%$ of respondents were not taking antihypertensive therapy The declared antihypertensive treatment highlights the frequent use of Ace Inhibitors $(36.4 \%)$, beta blockers (27.3\%), Calcium Antagonists ((21.2\%) Sartani (15.2\%) and diuretics $(6.1 \%) .55 \%$ of the respondents on treatment take 1 drug for hypertension, $25.9 \% 2$ drugs, $14.8 \% 3$ drugs and $3.7 \%$ more than 3 drugs. The subjects also all responded to control blood pressure at least once a month, having a device for self-measurement available in $81.8 \%$ of cases, contacting the doctor or pharmacist in the remaining $17.2 \%$ of cases. Among $82 \%$ of subjects who responded that they were hypertensive and under treatment, the degree of hypertension was normal high in $22 \%$ of cases, first degree in $29.6 \%$ and degree 2 in $40.7 \%$ of cases. After therapy, this distribution was high normal $33 \%$, First grade $33 \%$. Second degree $18.5 \%$, third degree $14.5 \%$. The last recorded measurement reported was $146.8 \pm 24.2 \mathrm{mmHg}$ for systolic and $89.4 \pm 18.8 \mathrm{mmHg}$ for diastolic. By applying the 130/85 $\mathrm{mmHg}$ cut-off to these blood pressure values to define optimal pressure control, it becomes evident that less than $30 \%$ of patients are in control.

Conclusions: The SIIA online tool represents a powerful aid for promoting the empowerment of the hypertensive patient. The data collection, however, must be further enhanced, so that it can be validated with respect to the personal data collection carried out on the occasion of the world days of arterial hypertension

\section{FEASIBILITY AND ACCEPTABILITY OF THE "AFA APP" EXERCISE PROGRAM TO REDUCE SEDENTARY BEHAVIOUR AND RELATED HEALTH ISSUES}

\author{
Guido Iaccarino, Danilo Contiero
}

Centro Interdipartimentale di Ricerca in Ipertensione Arteriosa e patologia correlate CIRIAPA, Federico II University, Naples, Italy

Introduction: Being physically active is a known determinant for healthy ageing, reducing the risk of almost all causes of death and morbidity. Nevertheless, adhere to physical exercise is still low and sedentary behaviour remains on of higher death risk factors among general population.

Aim: To verify whether the used ICT based tool for Physical Activity promotion increases adherence to Physical Activity and reduces sedentary behaviour. Furthermore, we assessed subjects of different age and sex, to evaluate if the AFA (Adapted Physical Activity) online exercise program proves to be effective as one of the first clinically tested online programs to involve remote adults in physical activity.

Methods: Participants were selected among those enrolled AFA clinic at the University Hospital Federico II. Patients underwent assessment of: weight, height, strength of lower and upper limbs, balance and flexibility. Based on the clinical assessment patients were 
proposed an Adapted Exercise Program. Patients could view the prescription on the dedicated Android App "AFA". The app allowed the team to have access to participants' exercise prescription's adherence. After 2 months, patients were reviewed at the clinic.

Results: This interventional study involved 72 historically (less than 2 hours per week of physical activity for the last 5 years) sedentary adults (50.0 $\pm 16,3$ years), 42 men and 30 women; 22 participants were active on the AFA platform, and 16 had an adherence to the program over the $50 \%$. Of the active participants, the average age was $52 \pm 15,5$ and the $64 \%$ was composed by men. The effects on the measured parameters after two months of AFA are recapitulated in the figure 1 .

Conclusions: Our results confirm that the ICT supported AFA protocol in feasible and it has a rate of success. Our results support the use of ICT based prescription of Physical Activity, in historical sedentary populations.

Figure 1 of Bari, Bari; ${ }^{g}$ Cardiology IV, “A.De Gasperi's” Department, Niguarda Ca' Granda Hospital, Milan; ${ }^{h}$ School of Medicine and Surgery, Milano-Bicocca University, Milan; ${ }^{i}$ Department of Clinical and Experimental Sciences, University of Brescia, Brescia; ${ }^{j}$ Istituto Auxologico Italiano, IRCCS, Department of Cardiovascular, Neural and Metabolic Sciences, San Luca Hospital, Milan; ${ }^{k}$ Department of Medicine and Surgery, University of Milan-Bicocca, Milan;

${ }^{l}$ Department of Internal Medicine, University of Genoa and IRCCS Ospedale Policlinico San Martino, Genoa; ${ }^{m}$ Department of Geriatric and Intensive Care Medicine, Careggi Hospital and University of Florence, Florence; ${ }^{n}$ Hospital S. Maria della Misericordia, Perugia, Italy; ${ }^{\circ}$ Department of Clinical and Experimental Medicine, University of Pisa, Pisa; ${ }^{p}$ Hypertension Unit, Division of Cardiology,

Department of Clinical and Molecular Medicine, Faculty of Medicine and Psychology, University of Rome Sapienza, Sant'Andrea Hospital, Rome; ${ }^{q}$ IRCCS Neuromed, Pozzilli; ${ }^{r}$ Clinica Medica, Department of
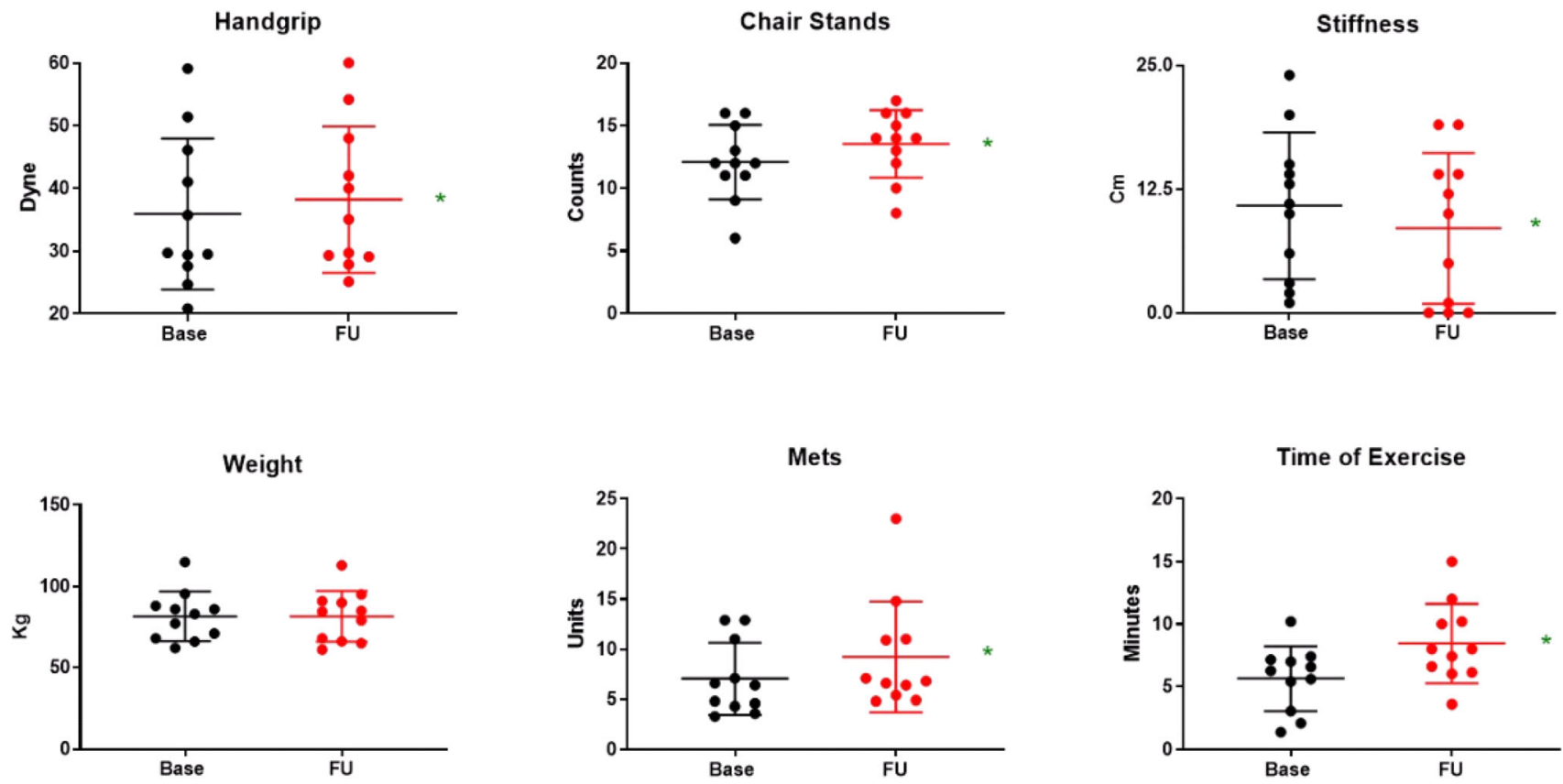

\section{STROKE AND SERUM URIC ACID: INTERACTION WITH AGE IN THE URRAH STUDY}

Valérie Tikhonoff ${ }^{\mathrm{a}}$, Edoardo Casiglia $^{\mathrm{b}}$, Massimo Cirillo $^{\mathrm{c}}$, Giovambattista Desideri ${ }^{\mathrm{d}}$, Claudio Ferri ${ }^{\mathrm{d}}$, Ferruccio Galletti ${ }^{\mathrm{e}}$, Loreto Gesualdo $^{\mathrm{f}}$, Cristina Giannattasio ${ }^{\mathrm{g}, \mathrm{H}}$, Guido Iaccarino ${ }^{\mathrm{e}}$, Maria Lorenza Muiesan $^{\mathrm{i}}$, Paolo Palatini ${ }^{\mathrm{b}}$, Gianfranco Parati ${ }^{\mathrm{j}, \mathrm{K}}{ }$, Roberto Pontremoli, Andrea Ungar ${ }^{\mathrm{m}}$, Paolo Verdecchia ${ }^{\mathrm{n}}$, Francesca Viazzi ${ }^{\mathrm{l}}$, Agostino Virdis $^{\mathrm{o}}$, Massimo Volpe ${ }^{\mathrm{p}, \mathrm{Q}}$, Guido Grassi ${ }^{\mathrm{r}}$ And Claudio Borghi ${ }^{\mathrm{s}}$. Working Group on Uric Acid and Cardiovascular Risk of the Italian Society of Hypertension (SIIA)

${ }^{a}$ Department of Medicine, University of Padua, Padua; ${ }^{b}$ Studium Patavinum, Department of Medicine, University of Padua, Padua; ${ }^{c}$ Department of Public Health, "Federico II" University of Naples, Naples; ${ }^{d}$ Department of Life, Health and Environmental Sciences, University of L'Aquila, L'Aquila; ${ }^{e}$ Department of Clinical Medicine and Surgery, "Federico II" University of Naples Medical School, Naples; ${ }^{f}$ Nephrology, Dialysis and Transplantation Unit, Department of Emergency and Organ Transplantation, "Aldo Moro" University

Medicine and Surgery, University of Milano-Bicocca, Monza; ${ }^{s}$ Department of Medical and Surgical Science, Alma Mater Studiorum University of Bologna, Bologna. Working Group on Uric Acid and Cardiovascular Risk of the Italian Society of Hypertension (SIIA)

INTRODUCTION: The Working Group on Uric Acid and Cardiovascular Risk of the Italian Society of Hypertension conceived and designed an ad hoc study aimed at searching for prognostic cut-off values of serum uric acid (SUA) in predicting combined (fatal and non-fatal) stoke events in the whole database.

AIM: The URRAH (URic acid Right for heArt Health) intends to clarify if SUA is associated with stroke, if a univariate cut-off level of SUA exists and can be confirmed being accepted in multivariate Cox regression models adjusted for confounders.

Methods: The URic acid Right for heArt Health (URRAH) study is a nationwide, multicentre, observational cohort study involving data on subjects aged 18 to 95 years recruited on a regional community basis from all the territory of Italy under the patronage of the Italian Society of Hypertension with a mean follow-up period of $120.7 \pm 61.8$ months. The receiver operating characteristic (ROC) curves method was used to search for prognostic cut-off of SUA for combined CBV events in the whole database 
Results: A total of 14, 588 subjects were included in the analysis. A prognostic cut-off value of SUA able to discriminate combined stroke events $(>4.79 \mathrm{mg} / \mathrm{dL}$ or $>284.91 \mu \mathrm{mol} / \mathrm{L})$ was identified by means of the ROC curve. Multivariate Cox regression analysis adjusted for confounders (age, sex, arterial hypertension, diabetes, chronic kidney disease, smoking habit, ethanol intake, body mass index, low-densitylipoprotein cholesterol and use of diuretics) identified an independent association between SUA and combined stroke events in the whole database (HR 1.249, 95\% confidence intervals, CI, 1.041-1.497, p = 0.016). The interaction term SUA $x$ gender was rejected from the Cox model while a positive interaction term was observed between SUA and age with a negative parameter estimates (beta $=-0.00813 \pm$ $0.00271, p=0.0027$ ). We therefore performed in all subjects a further multivariate Cox analysis having combined CBV events as dependent variable, and cut-off of SUA as independent variable, adjusting for the interaction term cuff-off SUA $x$ age and the confounders already mentioned. The interaction term was significantly accepted in the model (HR 0.974, 95\% CI 0.958-0.991, P = 0.003). We then calculated the HR with $95 \%$ CI of combined CBV events for cut-off value of SUA $>4.79 \mathrm{mg} / \mathrm{dl}$ by quintiles of age. In those subjects with SUA value over $4.79 \mathrm{mg} / \mathrm{dl}$, the $\mathrm{HR}$ of the combined CBV events tends to decrease with increasing age and disappears after 77 years of age (Figure 1).

Conclusions: The results of this study could explain the reported inconsistency on the relationship between the SUA level and strokes observed on several epidemiological studies.

Figure 1

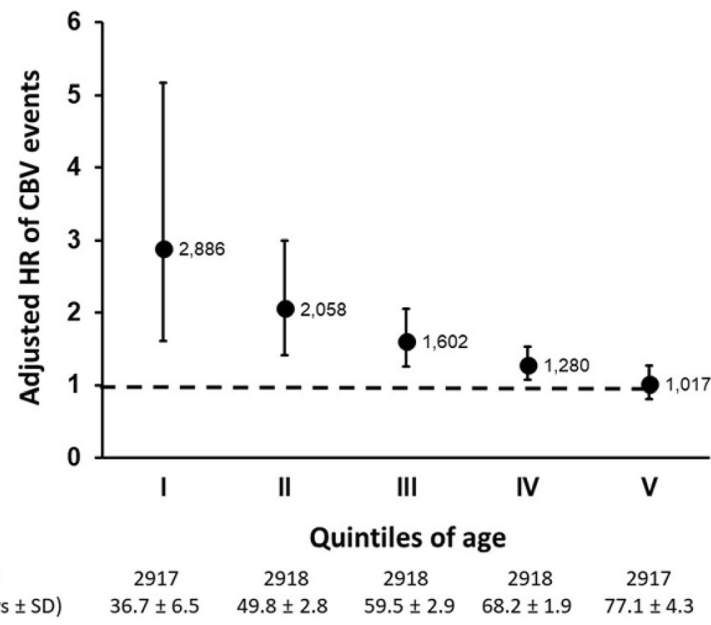

\section{Coronavirus and Epidemiology}

\section{HEART RATE AS A PROGNOSTIC MARKER OF SEVERITY OF SARS-COV-2 INFECTION: A RETROSPECTIVE OBSERVATIONAL STUDY}

\author{
Jennifer Vanoli ${ }^{1}$, Giacomo Marro ${ }^{1,2}$, Guido Grassi ${ }^{1,2}$, Fosca Quarti \\ Trevano $^{1,2}$, Dell'Oro Raffaella ${ }^{1,2}$ \\ ${ }^{1}$ University of Milano-Bicocca, Milan, Italy, ${ }^{2}$ Division of Medical \\ Clinic, San Gerardo Hospital, Monza, Italy
}

Introduction: SARS-CoV-2 infection has caused the death of 3, 73 million people worldwide. Several studies, including the SARS-RAS study conducted by SIIA, have shown that age and comorbidities are the main predictors of infection severity. The role of heart rate (HR), on the other hand, is still not entirely clear: tachycardia seems to be associated with a poor prognosis but few studies are available.

Aim: To evaluate how HR correlates with the outcome of SARSCoV-2 infection.

Methods: A cohort of 254 subjects positive for SARS-CoV-2 molecular swab admitted to the Medicine Department of the ASST San Gerardo Hospital in Monza was evaluated retrospectively. Clinical data were collected and infection outcomes recorded by identifying 4 classes of severity/intensity of care: (1) no need for oxygen support/need for low flow oxygen therapy, (2) need for high flow oxygen therapy/continuous positive airway pressure (3) transfer to the Intensive Care Unit (ICU) and (4) death. For analysis purposes, the subjects were selected for whom at least $3 \mathrm{HR}$ measurements were available before the start of the maximum intensity of care (137).

Results: The average age was 67 and $73 \%$ were men. 33 subjects (24\%) were categorized in severity class 1 while the remaining 104 in class $>1$, including 25 transfers to ICU and 45 deaths (43\%). The average age of the deceased was 77 years and had more comorbidities. For each class increase of maximum care intensity, an increase in mean HR was observed ( $79 \pm 9 \mathrm{bpm}$ vs $82 \pm 7 \mathrm{bpm}$ vs $85 \pm 9 \mathrm{bpm}$ vs $85 \pm 13 \mathrm{bpm}$ for mean HR of the first 3 days of hospitalization, p-value trend 0.0152). This correlation was maintained even after adjustment for temperature, blood pressure and $\mathrm{PaO} 2 / \mathrm{FiO} 2$ ratio, and correction for age, sex and comorbidities ( $\mathrm{p}$-value trend 0.0258 ). The same analysis was repeated for 77 subjects not receiving therapy with HR active drugs: even in this case, a correlation was observed between the increase in the average HR and the severity of the infection.

Conclusions: Our study shows that heart rate is an unfavourable prognostic marker during SARS-CoV-2 infection and this association holds true after adjustment for possible pharmacological interferences and main predictors of SARS-CoV-2 severity.

\section{Coronavirus and Hypertension}

\section{INCREASED HOME-OFFICE BLOOD PRESSURE DIFFERENCE AND RESTRAINED INDIVIDUAL WELL-BEING HIGHLIGHT A WORSE HYPERTENSIVE STATE DURING SOCIAL STRESS}

Pietro Nazzaro, Adele Nardecchia, Maria Contini, Gianfranco Amodio, Gabriella Schirosi, Lorenzo Debenedittis, Gabriella Aceto, Serena Calò, Mariangela Lisena, Gabriele Laselva, Irma Vitali, Graziano Servedio, Giulia Polieri, Maria Biallo

Dept. Biomedical Sciences and Human Oncology, Hypertension Unit "AM. Pirrelli", University of Bari "Aldo Moro”, Bari, Italy

Introduction: We previously showed that the increased home-office blood pressure difference (SBP/DBPhom-off) during a social stress phase (Covid lokdown) was associated to vascular damage and cognitive impairment.

Aim: To verify if SBP/DBPdom-off was still associated, in a larger cohort of 1015 grade- 1 hypertensives with similar age, hypertensive state, treatment and metabolic assessment, with vascular functional imparment and individual well being after 9-12 months, during the following pandemic phase of Covid-19.

Methods: During medical visit, the emotional (EMO) and physical (PHY) well being, by an adapted SF-36 questionnary, the cognitive impairment (COG-tot), by an 18-item survey, PWVcf and central (car) SBP, as arterial stiffness indices, were obtained in 196 patients 
with SBPhom-off $>10 \mathrm{mmHg}$ (H-HBP), in 542 with SBPhom-off \pm $10 \mathrm{mmHg}$ (E-HBP) and in 277 with SBPhom-off $<10 \mathrm{mmHg}$ (LHBP) by home blood pressure (HBP) taken 1-4 weeks before the visit. Results: Patients presented similar treatment compliance (Morisky), weight gain, quality/time of sleep, sleep apnea incidence (Lausanne NoSAS) and smoke habit, but they showed a different wellbeing due to hypertensive state (H-HBP: 3, $87 \pm 1,08 * * *$ vs $4,35 \pm 0,96$ e 4, $49 \pm 0,94)$ and treatment (H-HBP: 4, $28 \pm 1,04 * *$ vs $4,55 \pm 0,85 \mathrm{e}$ $4,61 \pm 0,81)$ and further characteristics $(\mathrm{m} \pm$ s.d.; $* \mathrm{p}<.05, * * \mathrm{p}<$ $.01,{ }^{* * *}: \mathrm{p}<.001$ vs H-HBP; ${ }^{\wedge}: \mathrm{p}<.05,{ }^{\wedge \wedge}: \mathrm{p}<.01,^{\wedge \wedge \wedge}: \mathrm{p}<.001$ vs E-HBP). autopsy of COVID-19 patients. However, no data are available about skin capillary alterations in these patients.

Aim: To investigate the microvascular alterations through capillaroscopy, in patients hospitalized for COVID-19, during the acute and after 3 months.

Method: We evaluated skin microvascular alterations in 22 patients admitted to Internal Medicine Department at Spedali Civili Hospital in Brescia and tested positive for a SARS-CoV-2 nasopharyngeal swab admission. Basal capillary density was performed by capillaroscopy bedside in the nailfold and the dorsum of the 4th finger.

\begin{tabular}{|c|c|c|c|c|c|}
\hline paz/var & SBPhom-off & SBP/DBPoff & SBPcar & PWVcf & COGtot \\
\hline $\mathrm{H}-\mathrm{HBP}$ & $20 \pm 9$ & $117 \pm 11 / 71 \pm 8$ & $116 \pm 11$ & $10,1 \pm 2,1$ & $29,8 \pm 10,2$ \\
\hline E-HBP & $1 \pm 5,5^{\star * *}$ & $128 \pm 11 / 76 \pm 9^{* * *}$ & $113 \pm 9^{* * *}$ & $9,1 \pm 2.3^{* \star \star}$ & $25,6 \pm 7,3^{\star \star \star}$ \\
\hline L-HBP & $-20 \pm 9^{* * * \wedge \wedge \wedge}$ & $142 \pm 14 / 82 \pm 9^{* * * \wedge \wedge \wedge}$ & $118 \pm 9^{\wedge \wedge \wedge}$ & $9,2 \pm 2,4^{* * *}$ & $25,2 \pm 7,8^{* * *}$ \\
\hline
\end{tabular}

the H-HBP patients showed a restrained EMO $\left(17,5 \pm 3,8\right.$ vs $19,3 \pm 3,5^{* * *}$ vs $\left.18,6 \pm 3,6^{* \wedge}\right)$ and PHYS $(9,1 \pm 2,3$ vs $9,8 \pm 2,1^{*}$ vs $10,2 \pm 1,9 * * * \wedge \wedge$ ) well-being. Furthermore, the change with BP measurements taken by nurses (SBPhom-lab: $-20 \pm 11$ vs $-6 \pm 10^{* * *}$ vs $8,03 \pm 13^{* * * \wedge \wedge \wedge}$ ) suggest that H-HBP e L-HBP might be assimilated to "masked htn" and "isolated clinic htn" phenomenom, respectively. Pearson test demostrated the association between SBPhom-off and SBPcar $\left(.384^{* *}\right)$ and PWVcf (.406***).

Conclusions: The findings highlight that the home BP and the perceived restrained emotional and physical well-being, during a social stress phase, might represent a useful sign of vascular damage and mild cpgnitive impairment although the office BP measurements are within the expected target.

\section{SKIN CAPILLARY ALTERATIONS IN PATIENTS WITH ACUTE SARS-COV-2 INFECTION}

Francesca Famà, Claudia Agabiti-Rosei, Paolo Malerba, Valeria Brami, Andrea Gaggero, Claudia Rossini, Matteo Nardin, Maria Lorenza Muiesan, Damiano Rizzoni Carolina De Ciuceis

Department of Clinical and Experimental Sciences, University of Brescia, Brescia, Italy

Introduction: Acute SARS-CoV-2 infection is associated with endothelial dysfunction and "endothelitis" which might explain systemic microvascular impairment. The presence of endothelial damage can promote vasoconstriction with consequent organ ischemia, inflammation, tissue edema, and a procoagulant state leading to increase in the incidence of cardio- and cerebro-vascular events. Microvascular thrombosis has been demonstrated in post-mortem
Capillaroscopy was repeated after 3 months from hospitalization for acute infection. Blood chemistry parameters and inflammatory markers were obtained at baseline and at the follow up.

RESULTS: Patients with COVID-19 showed skin microvascular complications such as thrombosis, microhemorrhages, and neoangiogenesis which were not detected after 3 months from the discharge (Figure 1A). A significant negative correlation between CRP and basal capillary density was observed in patients with acute SarsCov2 infection $(\mathrm{p}<0.05)$. A positive correlation between basal capillary density and lymphocyte number was detected. Moreover, a significant reduction of basal capillary density in the dorsum was observed after 3 months from the acute infection (Figure 1B).

CONCLUSION: This is an in vivo evidence of skin microvascular complications in patients with acute SarsCoV2 infection which supports the presence of endothelial dysfunction, inflammation, and thrombosis. Capillary alterations may reflect systemic vascular effects of viral infection. Moreover, a reduction of basal capillary density was observed after three months from acute infection probably due to acute inflammation and hypoxia which might have induced both vasodilation and angiogenesis.

Figure 1. Combined figure showing: patients complications at baseline and at 3 months (panel A); dorsal capillary number changes during the follow up period (panel B). 
A 10 Baseline

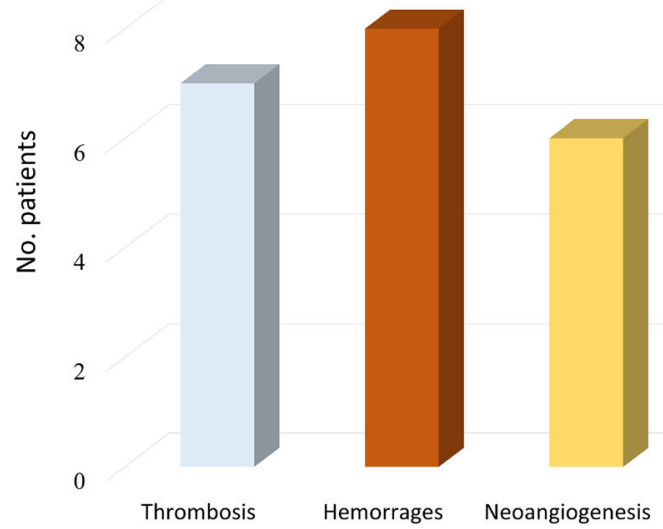

B

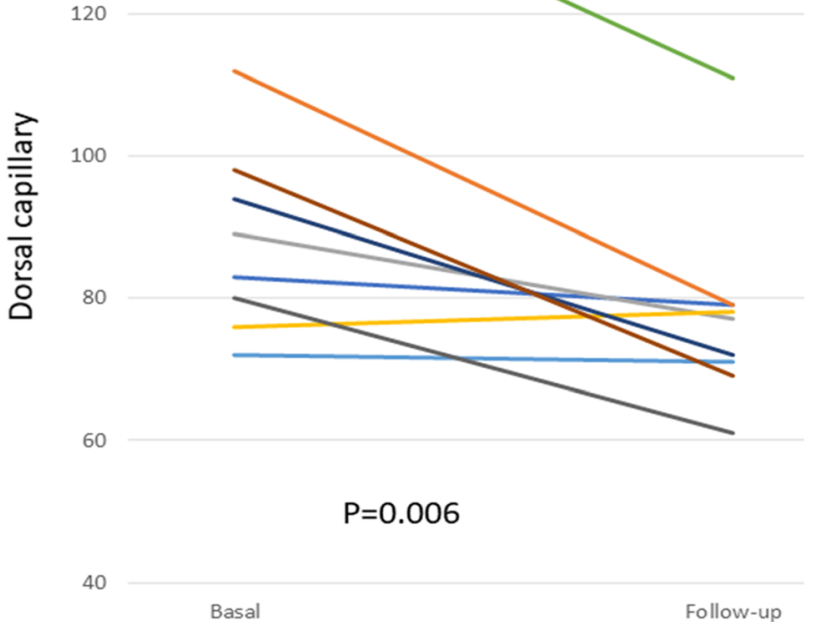

\section{ANALYSIS OF PROGNOSTIC FACTORS IN COVID- 19 HOSPITALIZED PATIENTS: AN ITALIAN SINGLE-CENTER PROSPECTIVE STUDY}

Antonio Concistrè, Cecilia Lina Pugliano, Luigi Petramala, Fabio Vinci, Ilaria Barchetta, Elio Pietro Perrone, Silvia Rella, Francesco Iannazzo, Alberta Angheloni, Lucia Coraggio, Francesco Di Rienzo, Daniela Maggi, Maurizio Muscaritoli, Claudio Letizia

Unit of Internal Medicine COVID-19, Policlinico Umberto I, Sapienza University, Rome, Italy

Introduction: COVID-19 clinical presentation ranges from asymptomatic infection to an inflammatory cytokine storm with multi-organ failure and fatal outcomes. The identification of high-risk patients for severe disease is crucial in order to plan an early treatment and intensive follow-up.

Aim: We aimed at investigating negative prognostic factors in a group of patients hospitalized for COVID-19. 3-months follow-up

Hemorrages Neoangiogenesis
Methods: 39 patients (19 men and 20 women, age $66.56 \pm 13.53$ years) were enrolled. Each patient received a work-up including medical history, clinical examination, arterial blood gas analysis, laboratory blood tests, feasible ventilatory support required during hospital stay, intensive care setting required, duration of illness and length of hospital stay ( $>$ or $<25$ days). For the assessment of the severity of COVID-19, three main indicators were considered: 1) the intensive care unit (ICU) admission 2) the hospitalization length $>25$ days; 3 ) the need of non-invasive ventilation (NIV).

Results: The independent risk factor associated with the ICU admission were lactic dehydrogenase elevation $(\mathrm{p}=0.046)$, C protein reactive elevation $(\mathrm{p}=0.014)$ at hospital admission and direct oral anticoagulant home therapy $(\mathrm{p}=0.048)$; for hospital length $>25$ days: early corticosteroid therapy $(\mathrm{p}=0.035)$; for NIV treatment: ferritin elevation at hospital admission $(\mathrm{p}=0.006)$.

Conclusions: the presence of the above factors may be useful to identify patients at high risk of developing a severe COVID-19 that need an early treatment and intensive follow-up. 


\section{Resistant Hypertension}

\section{DIAGNOSTIC EVALUATION \\ AND CARDIOVASCULAR RISK ASSESSMENT IN PATIENTS WITH HYPETENSIVE EMERGENCY-URGENCY}

Luigi Petramala, Antonio Concistrè, Priscilla Romagnoli, Francesco Circosta, Cristina Ruscitti, Cecilia Putigliano, Maurizio Soldini, Gino Iannucci, Claudio Letizia

Department of Traslational and Precision Medicine, University of Rome, Sapienza, Rome, Italy

Introduction: Hypertensive emergencies (EUI) represent an important cause of access to the emergency department (ED) (up to $10 \%$ ), involving patients with newly diagnosed arterial hypertension, or on antihypertensive therapy but poorly controlled blood pressure, and also patients with isolated blood pressure elevation.

Aim: evaluate the clinical and instrumental characteristics of patients with EUI, the prevalence of secondary forms of hypertension and asymptomatic organ damage.

Methods: we consecutively enrolled 53 patients $(28 \mathrm{M}, 25 \mathrm{D}$; average age $49 \pm 14 y y)$ sent to the Regional Center for the Study of Secondary Hypertension, University of Rome "Sapienza", after a previous visit for EUI at ED; patients underwent clinical, bio-humoral and instrumental evaluation for the study of subclinical organ damage (echocardiogram, carotid echo-color Doppler, fundus examination, 24-hour blood pressure monitoring-ABPM). Study for secondary forms of arterial hypertension has been performed in all patients.

Results: in $43 \%$ of patients arterial hypertension was diagnosed less than 3 months before, and in 30\% more than 12 months (mean duration $39 \pm 5$ months); as co-morbidities we observed: diabetes mellitus $6 \%$, OSAS $11 \%$, metabolic syndrome $23 \%$, dyslipidemia $28 \%$, hyperuricemia $34 \%$. The mean BMI was $27.7 \pm 5$ (obesity $30 \%$ and overweight $40 \%$ ). The outpatient blood pressure measurement showed values over $180 / 110 \mathrm{mmHg}$ in $10 \%$ of cases, while in $20 \%$ a well-controlled blood pressure $(<139 / 89 \mathrm{mmHg})$; in addition, the ABPM confirmed in $60 \%$ of cases a poor control of blood pressure values. In $60 \%$ of cases a subclinical organ damage previously not evidenced was found, and in particular $15 \%$ high $\mu$-albuminuria, $20 \%$ carotid atherosclerosis, $40 \%$ cardiac remodeling, $40 \%$ altered intimamedia thickness index, $40 \%$ altered fundus oculi, 50\% non-dipping pattern. The prevalence of secondary forms of arterial hypertension was $13 \%$, but up to $22 \%$ in patients with uncontrolled hypertension. Conclusions: EUIs represent a frequent cause of access to ED; such patients can be both hypertensive with isolated increase blood pressure behaviours, but also subjects already in therapy, with poorly controlled blood pressure, in which there is a high prevalence of clinically silent organ damage; it is important to evaluate the presence of secondary forms of hypertension as well as possible further comorbidities associated that can increase the overall cardiovascular risk.
Cerebrovascular Diseases

\section{CEREBROVASCULAR DAMAGE IN A MOUSE MODEL OF COGNITIVE DECLINE: AN ADVANCED NEUROIMAGING STUDY BY HIGH FIELD MAGNETIC RESONANCE IMAGING}

Lorenzo Carnevale $^{1}$, Raimondo Carnevale ${ }^{1}$, Francesco Mastroiacovo $^{1}$, Marialuisa Perrotta ${ }^{2}$, Daniela Carnevale ${ }^{1,2}$, Giuseppe Lembo $^{1,2}$

${ }^{I}$ Research Unit of Neuro and Cardiovascular Pathophysiology, IRCC Neuromed, Department of Angiocardioneurology and Translational Medicine, Pozzilli (IS), Italy, ${ }^{2}$ Department of Molecular Medicine, "Sapienza" University of Rome, Rome, Italy

Introduction: Hypertension in one of the main risk factors for the onset of vascular dementia $(\mathrm{VaD})$ and neurodegenerative pathologies as Alzheimer's Disease (AD). To identify the mechanisms underlying the development of these pathologies it is important to use welldesigned experimental models that recapitulate the pathophysiological characteristics of $\mathrm{AD}$ and $\mathrm{VaD}$ and let us investigate the molecular processes not explorable in humans. To ease the translational potential of these findings it is fundamental to characterize the cerebral brain damage with techniques and approaches which are easily applicable to the cognitive-declining patient, like the magnetic resonance imaging (MRI).

Aim: To this aim we subjected C57B1/6J mice to transverse aortic constriction (TAC), which induces a severe hypertension to the cerebral vasculature and leads in the long term to vascular cognitive impairment.

Methods: Two experimental groups were characterized by echocardiography and carotids analysis, behavioural phenotyping by Morris Water Maze (MWM) and Novel Object Recognition (NOR), cerebrovascular injury by macro and microstructural MRI in one group and cerebral blood flow in another group. T2-weighted volumetric images, 30-directions diffusion tensor imaging, arterial spin labelling sequences were performed on a murine MRI (Pharmascan 70/16, Bruker).

Results: TAC-subjected mice show a bilateral hypothalamic swelling at structural MRI and a white matter microstructural degradation in the Fimbria, evidenced by a reduction of Fractional Anisotropy. Hemodynamic alterations show a reduced cerebral blood flow and vascular rarefaction in the prefrontal cortex. TAC mice evidence a reduced learning capability, evidenced in the acquisition phase of MWM, and cognitive deficits in spatial and short-term memory, evidenced by probe trial in MWM and NOR test.

Conclusions: In conclusion we have setup and optimized an analysis pipeline for the cerebrovascular injury in experimental models of vascular cognitive impairment whose results are comparable and translatable to the clinical research. 
PREVALENCE OF CEREBROVASCULAR DYSPLASIA IN A COHORT OF PATIENTS WITH SPONTANEOUS CAROTID OR VERTEBRAL ARTERY DISSECTION

Pappaccogli Marco ${ }^{1}$, Fanelli Elvira ${ }^{1}$, Elisabetta Eula ${ }^{1}$, Fasano Chiara $^{1}$, Cerrato Paolo ${ }^{2}$, Bosco Giovanni ${ }^{2}$, Depaoli Alessandro ${ }^{3}$, Novelli Giulia $^{1}$, Veglio Franco ${ }^{1}$, Rabbia Franco ${ }^{1}$

${ }^{1}$ Division of Internal Medicine and Hypertension Unit, Department of Medical Sciences, University of Turin, Turin, Italy, ${ }^{2}$ Stroke Unit-AOU Città della Salute e della Scienza di Torino, Turin, Italy, ${ }^{3}$ Radiology Department-AOU Città della Salute e della Scienza di Torino, Turin, Italy

Introduction: Fibromuscular Dysplasia (FMD) is an idiopathic, segmental, noninflammatory and nonatherosclerotic disease of the musculature of arterial walls, leading to stenosis of small- and medium-sized arteries. FMD phenotype also includes aneurysms, arterial dissections and arterial tortuosity and should be always suspected in the differential diagnosis of spontaneous arterial dissection occurring in young patients without other risk factors.

Aim: The aim of this study was to assess the prevalence of cerebrovascular FMD in a cohort of patients admitted to the Stroke Unit with a diagnosis of spontaneous cerebrovascular arterial dissection (CeAD).

Methods: Imaging and clinical data of all patients with a diagnosis of CeAD discharged from the Stroke Unit of A.O.U. Città della Salute e della Scienza of Turin from 2016 to 2020 were retrieved and reevaluated. FMD diagnosis was assessed in the presence of at least a multifocal stenosis of the vascular beds explored by CTA, MRA or catheter-based angiography.

Results: Of 39 patients with spontaneous CeAD (79.5\% male; 51.7 ( \pm 8.5 ) y.o.), $36.1 \%$ of patient had hypertension and $51.5 \%$ were past or current smokers. In the context of CeAD the following arteries were the most frequently involved: left extracranic ICA (53.2\%), right extracranic ICA $(35.9 \%)$, left intracranic ICA (53.2\%), right intracranic ICA $(15.4 \%)$, left and right vertebral arteries $(12.9 \%$ and $5.1 \%$, respectively), and right CCA $(2.6 \%)$. Finally, two patients showed bilateral dissection $(5.1 \%)$.

When angiographic images were re-evaluated, 16 patients (41\%) were found to have FMD-related multifocal stenosis in the arteries explored (1/16 left extracranic ICA; 9/16 right ICA; 9/16 left ICA; $2 / 16$ bilateral ICA). Five patients showed arterial tortuosity in the cervical arteries and 2 patients developed a pseudoaneurysm in the context of the arterial dissection segment.

Finally, one patient showed a multifocal stenosis of the right femoral artery and another patient showed a left common iliac artery ectasia.

Conclusions: The results of this retrospective study confirm recent data from the international registries of FMD patients underlying that FMD is a common cause of spontaneous cerebrovascular arterial dissection, especially affecting young normotensive smoking males. Therefore, FMD should always be taken in consideration in the differential diagnosis of spontaneous arterial dissection or aneurysm rupture, especially if occurring in young subjects without major cardiovascular risk factors.
Hormonal Mechanisms and Endocrine Hypertension

\section{PRIMARY ALDOSTERONISM AND ANTIPHOSPHOLIPID SYNDROME: JUST COINCIDENCE OR CAUSAL RELATIONSHIP?}

Aurelio Negro, Giorgia Prampolini, Davide Cunzi, Rosetta Imbarlina, Ermina De Cristofaro

Internal Medicine and Hypertension Center Ospedale Sant'Anna di Castelnovo ne' Monti, Reggio Emilia, Italy

Introduction: Primary hyperaldosteronism is the most common form of secondary hypertension. Antiphospholipid syndrome (APS) is an acquired thrombophilia characterized by arterial and venous thrombotic events and pregnancy complications. Adrenal failure is a complication of APS. Cases of APA (aldosterone producing adenoma) are much rarer.

Clinical Case: 50 years old woman. Psychologist. No pregnancies. In September 2020 she presented to the emergency room with right upper limb paraesthesia and motor impairment. On admission the blood pressure was $170 / 100 \mathrm{mmHg}$; the patient had no apparent history of hypertension. Initial brain CT scan reported hypodense lesion in subcortical white matter of left parietal lobe (chronic ischemic lesion). Serum creatinine was $0.76 \mathrm{mg} / \mathrm{dl}$. Serum potassium was $3.6 \mathrm{mEq} / \mathrm{L}$. Antihypertensive therapy with ramipril, amlodipine and bisoprolol was initiated. The subsequent CT scan showed centimeter hypodense lesion in right semioval center-corona radiata (subacute ischemic stroke). The clinical course was favorable. The patient was discharged with antihypertensive drugs + ASA + atorvastatin. Laboratory tests came back positive for anti-cardiolipids antibodies IgM: 28, 2 MPL-U/ml (0,0-10,0), anti- beta 2-GP-I antibodies IgG: 5 $\mathrm{U} / \mathrm{ml}(0,0-4,9$ borderline), anti-beta 2 -GP-I antibodies IgM: 35, 3 $\mathrm{U} / \mathrm{ml}(0,0-4,9)$. Diagnosis of APS was confirmed (triple positive APL, stroke at young age). Angio TC abdomen: nothing to the vessels; left adrenal gland nodule. Our evaluation: we confirmed the previous anamnestic data. The patient reported home blood pressure values from one years of about $145 / 90 \mathrm{mmHg}$, which she considered normal; she reports for some times asthenia and muscle fatigue. After normalization of serum potassium $(4.1 \mathrm{mEq} / \mathrm{L})$ and pharmacological washout (only amlodipine was administered), renin and aldosterone

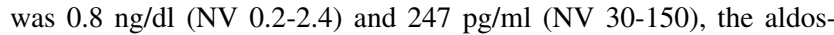
terone/renin ratio was suggestive for hyperaldosteronism and the probability of having an aldosteronoma was $98 \%$ according to ARR app. The post-saline load plasma aldosterone level was $93 \mathrm{pg} / \mathrm{ml}$. Adrenal vein sampling (without cosyntropin stimulation) showed left lateralization with IL 3.7. GRA analysis is in progress. The patient is waiting for left adrenalectomy. She is under treatment with eplerenone + amlodipine.

Conclusions: we presented a singular case of secondary arterial hypertension caused by primary hyperaldosteronism-likely do to APA-in a patient with triple positive antiphospholipid antibodies (APL). The potential role of APL in angiogenesis it's known, as these can induce-among the other things-VEGF expression. VEGF is a potent aldosterone secretagogue. In this case we can therefore hypothesize a stimulus to adrenal tumorigenesis induced by APL. 


\section{DEVELOPMENT AND VALIDATION OF A PREDICTION SCORE FOR THE DIAGNOSIS OF PRIMARY ALDOSTERONISM IN PATIENTS WITH A POSITIVE SCREENING TEST}

Jacopo Burrello $^{1}$, Martina Amongero ${ }^{2}$, Fabrizio Buffolo ${ }^{1}$, Elisa Sconfienza $^{1}$, Vittorio Forestiero ${ }^{1}$, Alessio Burrello ${ }^{3}$, Christian Adolf ${ }^{4}$, Laura Handgriff ${ }^{4}$, Martin Reincke ${ }^{4}$, Franco Veglio ${ }^{1}$, Tracy Ann Williams $^{1,}{ }^{4}$, Silvia Monticone ${ }^{1}$, Paolo Mulatero ${ }^{1}$

${ }^{I}$ Division of Internal Medicine and Hypertension, Department of Medical Sciences, University of Turin, Turin, Italy, ${ }^{2}$ Department of Mathematical Sciences G. L. Lagrange, Polytechnic University of Turin, Turin, Italy, ${ }^{3}$ Department of Electrical, Electronic and Information Engineering "Guglielmo Marconi" (DEI), University of Bologna, Bologna, Italy, ${ }^{4}$ Medizinische Klinik und Poliklinik IV, Klinikum der Universität, Ludwig-Maximilians-Universität München, Munich, Germany

Introduction: According to international guidelines, the management of patients with primary aldosteronism (PA) comprises screening and confirmation steps. Confirmatory testing is expensive, time-consuming, and there is no consensus on thresholds and tests to be used. Prediction models to avoid case confirmation may be useful for the diagnostic work-up of patients with PA.

Aim: The aim of the study was to develop and validate diagnostic algorithms to confirm or exclude the diagnosis of PA in patients with a positive screening test.

Methods: We enrolled 1, 024 subjects who underwent confirmatory tests for PA and were randomized to a training cohort $(\mathrm{N}=522)$ and to an internal validation cohort $(\mathrm{N}=174)$, to respectively build and test the diagnostic models. An independent external prospective cohort $(\mathrm{N}=328)$ was used to further validate the models. Four machine-learning based models and a 16-point simple scoring system were developed to discriminate patients with a confirmed diagnosis of PA.

Results: Six parameters were associated to a confirmed diagnosis of PA: male sex, antihypertensive medication, plasma renin activity, aldosterone, potassium levels and presence of organ damage. Learning algorithms displayed an accuracy ranging between 72.9 and $83.9 \%$, while the numerical score correctly classified $84.1 \%$ at training and $83.9 \%$ or $81.1 \%$ at internal and external validation, respectively. Integrating the score in a flow chart to select patients for confirmatory testing, we correctly managed all the patients, while reducing of $22.8 \%$ the number of confirmatory tests.

Conclusions: The application of integrated computational algorithms in clinical practice could improve the management of patients with PA by circumventing potentially unnecessary confirmatory tests.

\section{PREDICTION OF HYPERALDOSTERONISM SUBTYPES IN PATIENTS WITH UNILATERALLY SUCCESSFUL ADRENAL VEIN SAMPLING AND CONTRALATERAL SUPPRESSION}

Jacopo Burrello $^{1}$, Alessio Burrello ${ }^{2}$, Jacopo Pieroni ${ }^{1}$, Elisa Sconfienza $^{1}$, Vittorio Forestiero ${ }^{1}$, Martina Amongero ${ }^{3}$, Denis Rossato $^{4}$, Franco Veglio ${ }^{1}$, Tracy Ann Williams ${ }^{1,5}$, Silvia Monticone ${ }^{1}$, Paolo Mulatero ${ }^{1}$

${ }^{1}$ Division of Internal Medicine and Hypertension, Department of Medical Sciences, University of Turin, Turin, Italy, ${ }^{2}$ Department of Electrical, Electronic and Information Engineering "Guglielmo
Marconi" (DEI), University of Bologna, Italy, ${ }^{3}$ Department of Mathematical Sciences G. L. Lagrange, Polytechnic University of Turin, Turin, Italy, ${ }^{4}$ AOU Città della Salute e della Scienza-Service of Radiology, Turin, Italy, ${ }^{5}$ Medizinische Klinik und Poliklinik IV, Klinikum der Universität, Ludwig-Maximilians-Universität München, Munich, Germany

Introduction: The gold standard to discriminate patients with unilateral or bilateral forms of primary aldosteronism (UPA or BPA) is adrenal venous sampling (AVS). This procedure is technically challenging, and the results are not always interpretable in cases of unsuccessful cannulation of adrenal veins.

AIM: We aimed to develop diagnostic models to discriminate UPA from BPA, in patients with unilateral successful AVS in case of contralateral suppression of aldosterone production.

Methods: We retrospectively evaluated 158 patients referred to our tertiary hypertension unit to perform AVS: 110 patients were randomly assigned to a training cohort and 48 to a validation cohort, in order to respectively build and test the diagnostic models. Supervised learning algorithms and regression analysis were used to develop two prediction models and a 19-point score to discriminate patients according to subtype diagnosis.

Results: The following parameters were associated to UPA and were included in the diagnostic models: lowest potassium, aldosterone measurement at screening and after confirmatory testing, ipsilateral and contralateral imaging by $\mathrm{CT}$ scanning, and contralateral ratio (CLR) at AVS. Learning algorithms correctly discriminated the majority of patients both at training and validation of the models (accuracy ranging between 82.9 and $95.7 \%$ ). The numerical score displayed an AUC of 0.971 , with a sensitivity of $95.2 \%$ and a specificity of $96.9 \%$. The score was integrated in a flow-chart which correctly managed all patients except 3 (accuracy of 98.1\%), potentially avoiding the repetition of $77.2 \%$ of AVS procedures.

Conclusions: Learning algorithms could be used to integrate clinical and biochemical parameters, CT scanning, and CLR, in order to interpret AVS and define the subtype diagnosis in case of unsuccessful cannulation of one adrenal vein.

\section{DEVELOPMENT AND VALIDATION OF PREDICTION MODELS TO DISTINGUISH BETWEEN UNILATERAL AND BILATERAL FORMS OF PRIMARY ALDOSTERONISM}

Jacopo Burrello ${ }^{1}$, Alessio Burrello ${ }^{2}$, Jacopo Pieroni ${ }^{1}$, Elisa Sconfienza $^{1}$, Vittorio Forestiero ${ }^{1}$, Paola Rabbia ${ }^{3}$, Christian Adolf ${ }^{4}$, Martin Reincke ${ }^{4}$, Franco Veglio ${ }^{1}$, Tracy Ann Williams ${ }^{1,4}$, Silvia Monticone $^{1}$, Paolo Mulatero ${ }^{1}$

${ }^{I}$ Division of Internal Medicine and Hypertension, Department of Medical Sciences, University of Turin, Turin, Italy, ${ }^{2}$ Department of Electrical, Electronic and Information Engineering "Guglielmo Marconi" (DEI), University of Bologna, Italy, ${ }^{3}$ Division of Radiology, University of Turin, Turin, Italy, ${ }^{4}$ Medizinische Klinik und Poliklinik IV, Klinikum der Universität, Ludwig-Maximilians-Universität München, Munich, Germany

Introduction: Primary aldosteronism (PA) comprises unilateral (UPA) and bilateral forms (BPA). The diagnosis of UPA is important to recommend curative surgical intervention. The gold standard for PA subtyping is adrenal venous sampling (AVS), but this procedure is available only in few referral centers.

Aim: The aim of the study was to build prediction models for PA subtyping on the base of clinical and biochemical characteristics of patients. 
Methods: We recruited 333 patients referred to two tertiary hypertension units. Diagnostic models were respectively built and tested in a training $(\mathrm{N}=150)$ and in an internal validation cohort $(\mathrm{N}=65)$ from Torino. The models were then validated in an external independent cohort $(\mathrm{N}=118)$ from Munich. Logistic regression and supervised learning algorithms were used to develop a 20-point scoring system and two diagnostic models to classify patients with PA according to their subtype diagnosis.

Results: Aldosterone at screening and after confirmatory testing, lowest potassium value, presence/absence of nodules, nodule diameter, and computed tomography results were associated to UPA and included in the diagnostic models. Machine learning based models displayed a high accuracy both at training and internal validation (79.1\% to 93\%), while the 20-point score displayed an AUC of 0.896 (sensitivity $91.7 \%$; specificity $79.3 \%$ ). The external independent validation confirmed a reliable performance (accuracy ranging between 78.8 and $80.5 \%$ ). A flow-chart integrating the numerical score correctly managed $96.3 \%$ of patients and potentially avoided AVS in $43.7 \%$ of cases.

Conclusions: Computational modelling can be integrated in the management of patients with PA to define the subtype diagnosis and guide surgical decision in centers where AVS is not available.

\section{CLINICAL DATA AND 11BHSD2 EXOSOMAL EXPRESSION IN THE FOLLOW-UP OF TWO BROTHERS WITH APPARENT MINERALOCORTICOID EXCESS SYNDROME}

Mariangela Veneri, Domenica De Sanctis, Annalisa Castagna, Simonetta Friso, Silvia Udali, Francesca Morandini, Oliviero Olivieri, Francesca Pizzolo

Department of Medicine, Unit of Internal medicine, University of Verona, Italy

Introduction: Apparent Mineralocorticoid Excess (AME) is a hereditary autosomal recessive disorder caused by the 11-hydroxysteroid dehydrogenase type 2 (11-HSD2) enzyme deficiency with consequent activation of the mineralocorticoid receptor not mediated by aldosterone.

The 11-HSD2 is a microsomal enzyme that convert the active steroids cortisol and corticosterone into their inactive metabolites, cortisone and 11-dehydrocorticosterone, respectively. A deficient enzymatic function leads to an impaired conversion of cortisol into cortisone. Both aldosterone and cortisol are ligands for the mineralocorticoid receptor (MR), but blood concentrations of cortisol are several fold higher than those of aldosterone. If 11HSD2 is defective, more cortisol is therefore available to MR binding, resulting in a higher MR activation. The elevated intracellular cortisol brings to the mineralocorticoid receptor activation resulting in the typical clinical features of hypertension and hypokalemia in reason

of the renal sodium reabsorption and potassium excretion. The activity of the $11 \beta-H S D 2$ enzyme can be estimated by measuring either the urinary cortisol metabolites ratio (THF+5 $\alpha \mathrm{THF} / \mathrm{THE})$. AME is a rare disease, with less than 100 cases described in the literature, associated to less than overall 50 mutations described at the HSD11B2 gene site. Urinary extracellular vesicles and especially urinary exosomes, are secreted by renal tubular epithelial cells and carry nucleic acids, proteins and lipids; they can be easily detected in urine and provide diagnostic and pathophysiological information without an invasive tissue biopsy. We have recently documented, on 8 patients with A221G mutation $(662 \mathrm{C}>\mathrm{G})$, that the exosomal expression of HSD11B2 mRNA varied according to the $662 \mathrm{C}>\mathrm{G}$ genotype (the homozygous probands showed the highest, the heterozygous subjects intermediate and the wild-type subjects the lowest levels) and among heterozygous carriers, hypertensive subjects had higher HSD11B2 exosomal mRNA than normotensives. The exosomal expression was also inversely related with the renin and aldosterone concentrations and positively related with the ratio (THF +5 alfaTHF/THE). The aim of this study is to describe the clinical and biochemical follow up in two brothers with AME after the introduction of targeted medical therapy.

Aim: The aim of this study was to value the clinical performance of two brothers affected by AME and the possible variation of the HSD11B2 gene exosomal expression after the introduction of targeted therapy.

Materials: Two brothers (14 and 7 years old) with AME, homozygous for the GG allele, were enrolled in the study. The older patient was treated with eplerenone, ACE inhibitor and amiloride/hydrochlorothiazide; the younger brother was treated with eplerenone, ACE inhibitor and calcium channel blocker. Both blood pressure values, organ damage, plasma potassium and creatinine values were monitored over time. Urinary steroids ratios and the exosomal expression of HSD11B2 mRNA were measured before the introduction of the therapy (T0) and after 5 years. Urinary exosomes were extracted from morning urine and exosomal HSD11B2 mRNA was quantified by Droplet Digital PCR (ddPCR). $\beta-2$ microglobulin gene was selected as reference housekeeping gene.

Results: Before therapy introduction the two boys displayed the tipical fenotype of the disease with low aldosterone, renin and $\mathrm{K}$ and high blood pressure with signs of hypertensive heart disease (only in the older brother), and the exosomal expression of 11bHSD2 was quantified. During antihypertensive therapy (including eplerenone, a MR receptor antagonist) biochemical parameters improved from baseline, with a better blood pressure control and normalization of renin, aldosterone and kalaemia values and regression of hypertensive heart disease. Moreover, a net reduction in exosomal HSD11B2 mRNA was observed over time. If HSD11B2 gene expression at baseline was increased in order to compensate the excessive MR activation, a therapy mediated MR inhibition, effectively reduced blood pressure but also HSD11B2 mRNA expression. In line with this hypothesis, the higher THF+aTHF/THE ratio as compared to baseline observed after eplerenone treatment in the two boys is consistent with the finding of a lower exosomal HSD11B2 gene expression.

Conclusions: The identification of disease and the introduction of targeted therapy, in patients with AME, allows to improve blood pressure control and regression of organ damage, making the longterm prognosis more favorable. This study confirms the correlation between the improvement of the clinical status and biochemical parameters, and shows, for the first time, a lower exosomal HSD11B2 gene expression after the introduction of specific therapy. This is a further indirect demonstration of the role of a gene expression modulation of HSD11B2 related to the entity of MR activation.

\section{URINARY EXTRACELLULAR VESCICLES PROTEOMICS IN PRIMARY ALDOSTERONISM}

Lorenzo Bertolone ${ }^{1}$, Annalisa Castagna ${ }^{1}$, Marcello Manfredi ${ }^{2}$, Domenica De Santis ${ }^{1}$, Francesca Ambrosani ${ }^{1}$, Paolo Mulatero ${ }^{3}$, Mariangela Veneri ${ }^{1}$, Oliviero Olivieri ${ }^{1}$, Francesca Pizzolo ${ }^{1}$

${ }^{1}$ Department of Medicine, Unit of Internal Medicine, University of Verona, Verona, Italy, ${ }^{2}$ Department of Translational Medicine, University of Piemonte Orientale, Novara, Italy, ${ }^{3}$ Internal Medicine and Hypertension Division, Department of Medical Sciences, University of Turin, Turin, Italy 
Introduction: Urinary extracellular vesicles and especially urinary exosomes (UE), are secreted by renal tubular epithelial cells and carry nucleic acids, proteins and lipids; they can be easily detected in urine and provide diagnostic and pathophysiological information without the need of an invasive tissue biopsy. Exosomes are involved in extracellular trafficking, differentiation and survival, and can be produced by all cell types. Recently they have been considered as a valuable tool also in the field of hypertension research and primary aldosteronism (PA).

Methods: Patients with essential hypertension $(\mathrm{EH})$ and PA were enrolled in the study $(\mathrm{EH}=12, \mathrm{PA}=23: 11 \mathrm{BAH}$ and $13 \mathrm{APA})$. Clinical and biochemical parameters were available for all the subjects involved. UEV were isolated from urine by ultracentrifugation and analysed by Trasmission electron microscopy (TEM). The protein content of UEV was assessed through an untargeted MS-based approach. Statistical analysis was performed to get prospects biomarker (T-test, fold-change, etc.). Network analysis was carried out on the significantly different identified proteins to gain insight into the pathways involved in the definition of the pathology.

Results: MS analysis provided more than 300 proteins identification. Exosomal markers such as CD9 and CD63 were detected in all the samples. Several biomarker candidates separating EH and PA patients as well as BAH and APA subtypes resulted after statistical elaboration and filtering of the results. Proteins involved in biological pathways such as positive regulation of immune response, receptormediated endocytosis, renal water transport and cell activation involved in immune response were found. In particular, some key proteins involved in water reabsorption mechanisms, such as AQP1 and AQP2, were among the best biomarkers candidates for discriminating $\mathrm{EH} v s$ PA.

Conclusions: The differences observed in UEVs protein composition supported the role of a specific regulation in water absorption mechanisms between PA and EH. Through this proteomic approach in UEV we have identified several molecular markers that can improve the PA diagnostic work-up and gain insight on the pathophysiological features of this disease. Further studies in this research area would be highly beneficial to improve the molecular characterization of complex diseases such as hypertension.

\section{QUALITY OF LIFE IN PRIMARY ALDOSTERONISM BEFORE AND AFTER TREATMENT: A PROSPECTIVE OBSERVATIONAL STUDY}

Fabrizio Buffolo ${ }^{1}$, Giovanni Cavaglià ${ }^{1}$, Jacopo Burrello ${ }^{1}$, Martina Amongero $^{2}$, Martina Tetti ${ }^{1}$, Alessio Pecori ${ }^{1}$, Elisa Sconfienza ${ }^{1}$, Franco Veglio $^{1}$, Paolo Mulatero ${ }^{1}$, Silvia Monticone ${ }^{1}$

${ }^{1}$ Division of Internal Medicine and Hypertension Unit, Department of Medical Sciences, University of Turin, Turin, Italy, ${ }^{2}$ Department of Mathematical Sciences G. L. Lagrange, Polytechnic University of Turin, Turin, Italy

Introduction: Some studies suggested that the quality of life (QOL) of patients with primary aldosteronism (PA) is impaired compared to the general population. However, no study performed a direct comparison between patients with essential hypertension (EH) and patients with PA, nor a comparison of the effect on QOL of specific treatment for PA and general antihypertensive treatment.

Aim: We designed a prospective observational study to compare the QOL of patients with PA to the QOL of patients with $\mathrm{EH}$, before and after treatment.
Methods: We prospectively recruited 70 patients affected by PA and 70 patients with $\mathrm{EH}$, matched for age, sex, blood pressure levels and intensity of anti-hypertensive treatment. QOL was assessed by 36-Item Short Form Health Survey (RAND SF-36) at baseline and after 2 and 6 months of specific treatment for PA (surgical adrenalectomy or mineralocorticoid receptor antagonists, MRA) or 6 months after optimization of medical therapy for EH, without MRA. Results: Compared with the general healthy population, the QOL of patients with PA was significantly impaired, but not significantly different from the QOL of matched patients with EH. Both unilateral adrenalectomy and treatment with MRA allowed an improvement of QOL in patients with PA, that was more pronounced after surgical approach. In patients with $\mathrm{EH}$, optimization of antihypertensive treatment (without MRA) resulted in a minimal improvement in only one of eight domains, in patients with $\mathrm{EH}$.

Conclusions: The QOL of patients with PA is impaired in both unilateral and bilateral forms. The reduction in QOL is likely caused by the effects of intensive anti-hypertensive treatment and uncontrolled blood pressure, more than direct effect of aldosterone excess. Surgical and medical treatment for PA significantly improves QOL, by reduction of anti-hypertensive treatment, after adrenalectomy, and improvement of blood pressure control in both medical and surgical approach.

\section{A SYSTEMATIC REVIEW AND META-ANALYSIS OF DIAGNOSTIC GAIN OF EXCLUSION TESTS OVER BASELINE ARR FOR THE DETECTION OF UNILATERAL PRIMARY ALDOSTERONISM}

\author{
Rui Zhu, Tungalatamir Shagjaa, Brasilina Caroccia, Giacomo \\ Rossitto, Teresa Maria Seccia, Gian Paolo Rossi
}

Clinica dell'Ipertensione Arteriosa, Department of MedicineDIMED, University of Padua, Padua, Italy

Introduction: To avoid costly and invasive lateralization procedures in patients with a false-positive aldosterone-to-renin ratio (ARR) result current guidelines recommend one or more exclusion tests. However, being examined only in few studies to date, the diagnostic gain of these tests over the baseline ARR, remains uncertain.

Aim: To meta-analyze available studies in order to determine the diagnostic accuracy and gain of exclusion tests in the work-up of PA. Methods: We searched the PubMed, EMBASE, Web of Science, and the Cochrane databases for relevant studies published over 50 years. Cohort studies assessing the diagnostic performance for identifying unilateral PA of the ARR and the exclusion tests were selected using if they used the gold standard of biochemical cure after surgery, or at least a golden standard (adrenal venous sampling or pathology or blood pressure fall after surgery) as reference. A modified QUADAS2 criteria was used to assess the quality of included studies.

Study data examined included the summary receiver operating characteristic (SROC) curve and corresponding area under the curve (SAUC), pooled sensitivity and specificity, diagnostic odds ratios (DOR).

Results: 20 articles entailing 29 separate studies fulfilled the predefined inclusion criteria and could be included. Of them, 11 studies evaluated the diagnostic accuracy of baseline ARR, 8 of captopril challenge test (CCT), 9 of saline infusion test (SIT) and 1 of furosemide upright test. The pooled sensitivity, specificity, diagnostic odds ratio (DOR) and SAUC of baseline ARR, CCT and SIT (Table), showed no differences of diagnostic accuracy between baseline ARR, CCT and SIT (all p NS); no solid conclusion could be drawn for FUT due to the scant data. 
Conclusions: the systematic use of CCT and SIT is not justified as they furnished no significant diagnostic gain over a carefully performed ARR for diagnosing unilateral PA.

\begin{tabular}{|l|l|l|l|l|}
\hline & $\begin{array}{l}\text { Sensitivity } \\
(\%)\end{array}$ & Specificity (\%) & DOR & SAUC \\
\hline Baseline ARR & $85(80-90)$ & $91(90-92)$ & $60(27-132)$ & $\begin{array}{l}0.94(0.85- \\
0.96)\end{array}$ \\
\hline CCT & $78(73-82)$ & $84(82-85)$ & $40(13-128)$ & $\begin{array}{l}0.90(0.83- \\
0.95)\end{array}$ \\
\hline SIT & $93(90-96)$ & $86(83-89)$ & $128(42-368)$ & $0.95(0.90-$ \\
& & & & $0.97)$ \\
\hline
\end{tabular}

\section{EFFECTS OF MINERALOCORTICOID RECEPTOR ANTAGONISTS ON ALDOSTERONE TO RENIN RATIO IN PATIENTS WITH PRIMARY ALDOSTERONISM}

Alessio Pecori ${ }^{1}$, Fabrizio Buffolo ${ }^{1}$, Jacopo Burrello ${ }^{1}$, Giulio Mengozzi $^{2}$, Francesca Rumbolo ${ }^{2}$, Valeria Avataneo ${ }^{3}$, Antonio $D^{\prime} A_{v}$ lio $^{3}$, Franco Rabbia ${ }^{1}$, Chiara Bertello ${ }^{1}$, Franco Veglio ${ }^{1}$, Paolo Mulatero $^{1}$, and Silvia Monticone ${ }^{1}$

${ }^{1}$ Division of Internal Medicine and Hypertension Unit, Department of Medical Sciences, University of Turin, Turin, Italy, ${ }^{2}$ Department of Laboratory Medicine, AOU Città della Salute e della Scienza, Turin, Italy, ${ }^{3}$ Laboratory of Clinical Pharmacology and Pharmacogenetics, Department of Medical Sciences, University of Turin, Amedeo di Savoia Hospital, Turin, Italy

Introduction: Current guidelines recommend to withdraw interfering antihypertensive drugs before to perform a screening test for primary aldosteronism (PA). Recent studies suggested that diagnostic work-up of PA might be performed without withdrawing mineralocorticoid receptor antagonist (MRA) treatment in selected patients, especially when severe hypertension and hypokalemia are difficult to control.

Aim: We designed a prospective study to evaluate the effects of MRA treatment on aldosterone to renin ratio (ARR) in patients with PA

Methods: We prospectively recruited 121 patients with confirmed PA and started on canrenone treatment. Eighteen patients were enrolled in a short-term treatment cohort to assess the effects of MRA on aldosterone to renin ratio (ARR) and potassium after 2 and 8 weeks of canrenone therapy. One hundred and two patients were recruited in the long-term treatment cohort to evaluate the effects of canrenone on ARR after 2 to 12 months of MRA therapy.

Results: Plasma renin activity and potassium displayed a significant increase, and ARR showed a significant reduction compared with baseline in patients with PA after MRA initiation. These effects were observed both after a short- and a long-term treatment with canrenone, and were progressively more significant after longer periods of therapy and with greater doses of MRA. We demonstrated that canrenone assumption severely impacts on the rate of false-negative screening tests for PA, which raised from $16.7 \%$ to $72.5 \%$ after 2 weeks and 7-12 months of MRA treatment, respectively.

Conclusions: ARR testing for PA screening should not be performed under MRA therapy.

\section{CORTISOL EXCESS IN PRIMARY ALDOSTERONISM: EVIDENCE AGAINST A PREVALENT “CONNSHING" SYNDROME}

Giacomo Rossitto, Giorgia Antonelli, Giulio Ceolotto, Viola Sanga, Brasilina Caroccia, Rhian M. Touyz, Mario Plebani, Christian Delles, Gian Paolo Rossi

${ }^{1}$ Clinica dell'Ipertensione, DIMED, University of Padua, Padua, Italy, ${ }^{2}$ Institute of Cardiovascular and Medical Sciences, University of Glasgow, Glasgow, United Kingdom, ${ }^{3}$ Laboratory Medicine, University of Padua, Padua, Italy

Introduction: Autonomous cortisol co-secretion was suggested to be prevalent in primary aldosteronism (PA). PA features excess glomerular filtration rate (GFR), or hyperfiltration.

Aim: Our study investigated cortisol excess in PA in relation to GFR, a determinant of cortisol excretion, and to the cortisol and aldosterone secretion by each adrenal gland.

Methods: Morning plasma and 24h-urinary free cortisol (PCC and UFC), and creatinine clearance were measured from patients with $\mathrm{EH}$ $(n=147)$ or PA $(n=25)$ at the time of screening for secondary hypertension under non-interfering medications, and from PA patients treated with a mineralocorticoid receptor antagonist or submitted to adrenalectomy ( $\mathrm{n}=13$ and 25$)$ at follow-up. In a large cohort of consecutive PA patients undergoing adrenal vein sampling (AVS) we assessed glucocorticoid circadian rhythmicity and calculated lateralisation indexes (LI) for both aldosterone and cortisol.

Results: 24h-UFC was higher in PA vs PH (78[42-106] nmol/d vs $56[33-83] \mathrm{nmol} / \mathrm{d}, \mathrm{p}<0.05)$ and was reduced by treatment $(36[28$ $70], \mathrm{p}<0.01)$, while PCC did not differ across groups $(\mathrm{p}=0.75)$. Creatinine clearance, surrogate for GFR, paralleled 24h-UFC trends (PH: 11.2[7.4-14.9], PA: 13.6[10.8-17.8], treated-PA: 9.5[7.5-13.8] $\mathrm{ml} / \mathrm{min}$ ); adjustment of UFC for creatinine clearance resulted in no difference across groups $(\mathrm{p}=0.65)$.

In patients undergoing AVS, the slope of the regression line between the LIs for aldosterone and for cortisol did not differ from $0(\mathrm{p}=0.60)$ and revealed no co-secretion in 82/84 cases; circadian rhythmicity of ACTH and cortisol was preserved $(n=45)$.

Conclusions: Our results do not support the view of a prevalent "Connshing" syndrome. Excess cortisol excretion is likely explained by glomerular hyperfiltration. 


\section{Blood Pressure Measurement}

\section{ACCURACY OF THE OSCILLOMETRIC METHOD FOR THE MEASUREMENT OF HEART RATE AT REST AND DURING MILD EXERCISE}

\author{
Francesca Saladini ${ }^{1,2}$, Claudio Fania ${ }^{1}$, Paolo Palatini ${ }^{1}$ \\ ${ }^{1}$ Department of Medicine, University of Padua, Padua, Italy, \\ ${ }^{2}$ Cardiology Unit, Cittadella Town Hospital, ALSS 6 Euganea, Italy
}

Aim: The aim of the study was to determine the accuracy of an oscillometric device for the measurement of heart rate (HR) during rest and exercise.

Methods: The Microlife WatchBP O3, a monitor previously validated for blood pressure measurement, was tested in 36 subjects from the general population (mean age, 72.9 years; 18 men). The algorithm calculates the average interval of all pulse intervals during a measurement cycle. The number of pulse intervals taken into account may vary in relation to the individual HR and pulse pressure levels. Typically, with a deflation rate of $4 \mathrm{mmHg} / \mathrm{sec}, 10$ to 20 pulse intervals are averaged during a measurement cycle. HR was measured at rest and during stress testing on a cycle ergometer in order to increase HR by $15 \%$ or more. HR was computed from the oscillometric waves recorded during the deflation phase of the blood pressure measurement and was compared with HR measured by pulse palpation by two observers.

Results: At rest, the mean device-observer HR difference was $0.9 \pm$ $2.1 \mathrm{bpm}$. During exercise, the average HR increase was $20.3 \%$ and the mean device-observer difference was $0.6 \pm 2.6 \mathrm{bpm}$. The deviceobserver HR differences were all included within $\pm 6 \mathrm{bpm}$ both at rest and during exercise. A close correlation was found between HR measured by the observers and the device at rest $(r=0.978)$ and during exercise $(r=0.973)(p<0.0001)$. At rest, the device-observer HR difference was unrelated to HR measured by the observers and to pulse pressure. A lack of correlation of the device-observer HR difference with HR level and pulse pressure was also found during exercise.

Conclusions: The present study shows that the oscillometric device Microlife WatchBP O3 was able to provide reliable HR measurements during rest and mild exercise across all levels of HR and pulse pressure. Whether evaluation of HR performance should be included during validation testing of automatic monitors should be established by regulatory bodies.

\section{SHORT- AND LONG-TERM REPRODUCIBILITY OF NIGHTTIME BLOOD PRESSURE PHENOTYPES AND NOCTURNAL BLOOD PRESSURE REDUCTION}

Fosca Quarti Trevano ${ }^{1}$, Raffaella Dell'Oro ${ }^{1}$, Jennifer Vanoli ${ }^{1}$, Rita Facchetti $^{1}$, Cesare Cuspidi ${ }^{2}$, Giuseppe Mancia ${ }^{3}$, Guido Grassi ${ }^{1}$

${ }^{1}$ Clinica Medica, Department of Medicine and Surgery, University of Milano-Bicocca, Milan Italy, ${ }^{2}$ Department of Medicine and Surgery, University of Milano-Bicocca, Milan, Italy, ${ }^{3}$ Policlinico di Monza, Monza and University Milano-Bicocca, Milan, Italy

Introduction: In 1722 hypertensives recruited for the European Lacidipine Study on Atherosclerosis and treated with atenolol or lacidipine ( \pm additional drugs) during 4 years, we evaluated the longterm reproducibility of dipping, nondipping, extreme dipping, and reverse dipping.
Aim: This is an information of key relevance for defining the prognostic impact of these blood pressure (BP) phenotypes.

METHODS: Ambulatory BP was measured at baseline and every year during treatment, allowing repeated classifications of these conditions.

Results: Based on systolic BP, at baseline $50.1 \%, 37.5 \%, 4.5 \%$, and $7.9 \%$ were dippers, nondippers, extreme dippers, and reverse dippers, respectively. Antihypertensive drug treatment reduced the number of dippers and extreme dippers in favor of nondippers and reverse dippers. During treatment, the dipping and nondipping phenotypes were markedly unstable and shifts from one to other phenotypes were even more common in reverse and extreme dippers In only a very small fraction of patients, a given nighttime BP phenotype was persistently found throughout the 4 years. Data were similar for diastolic BP and in subgroups differing for the type of treatment, the treatment complexity, and the achieved on-treatment BP value Poor reproducibility characterized also the absolute nighttime $\mathrm{BP}$ reduction as measured by the nighttime BP fall and the night/day BP ratio.

Conclusions: Nighttime BP phenotypes are all largely inconsistent, their detection over the entire treatment period involving just a minute fraction of the entire patients' cohort.

\section{SYMPATHETIC NEURAL MECHANISMS UNDERLYING UNATTENDED AND ATTENDED BLOOD PRESSURE MEASUREMENT}

Fosca Quarti Trevano ${ }^{1}$, Gino Seravalle ${ }^{2}$, Rita Facchetti ${ }^{1}$, Raffaella Dell'Oro ${ }^{1}$, Jennifer Vanoli ${ }^{1}$, Gianluca Perseghin ${ }^{3}$, Giuseppe Mancia ${ }^{4}$, Guido Grassi ${ }^{1}$

${ }^{1}$ Clinica Medica, Department of Medicine and Surgery, University of Milano-Bicocca, Milan, Italy, ${ }^{2}$ Istituto Auxologico Italiano, San Luca, Milan, Italy, ${ }^{3}$ Department of Medicine and Rehabilitation, Policlinico di Monza, Monza, Italy, ${ }^{4}$ Policlinico di Monza, Monza and University Milano-Bicocca, Milan, Italy

Introduction: Blood pressure (BP) changes associated with unattended and attended BP measurements are thought to be mediated by neurogenic mechanisms.

Aim: In this study we tested this hypothesis by evaluating different dynamic markers of sympathetic activity such as muscle sympathetic nerve traffic (MSNA), skin sympathetic nerve traffic (SSNA) and heart rate (HR) before and during unattended and attended $\mathrm{BP}$ measurement.

Methods: This was done in 18 mild-to-moderate untreated essential hypertensive patients (age: $40.2 \pm 2.8$ years, mean \pm SEM), undergoing MSNA and SSNA recording, together with BP and HR, before and during automated BP evaluation in absence or in presence of a doctor.

Results: The BP reduction detected during unattended measurement was associated with a reduction in HR and SSNA and almost unchanged MSNA. Conversely during attended BP measurement there was an increase in BP, a slight HR and SSNA elevation and a slight MSNA decrease. The differences in BP, HR, MSNA and SSNA between unattended and attended BP measurement $(-9.3 \pm 1.1$ $\mathrm{mmHg},-7.3 \pm 1.0$ beats $/ \mathrm{min},+4.7 \pm 1.3$ bursts $/ \mathrm{min}$ and $-6.3 \pm 0.9$ bursts/min) were all statistically significant $(\mathrm{P}<0.01)$. The mean $\mathrm{BP}$ decrease observed during unattended BP measurement was significantly directly related to the concomitant SSNA and HR reductions ( $\mathrm{r}$ $=0.49 \mathrm{P}<0.05$ and $\mathrm{r}=0.69, \mathrm{P}<0.001)$ but not to MSNA changes $(\mathrm{r}$ $=-0.21, \mathrm{P}=\mathrm{NS}$ ). No relationship was found between the HR changes and the concomitant MSNA and SSNA modifications.

Conclusions: These data provide the first evidence that sympathetic mechanisms underpin the BP changes associated with unattended or 
attended BP measurement. They also show, however, that these sympathetic alterations are heterogeneous, combining excitation in one district with inhibition in the other.

\section{ADDING HOME AND/OR AMBULATORY BLOOD PRESSURE TO OFFICE BLOOD PRESSURE FOR CARDIOVASCULAR RISK PREDICTION}

Raffaella Dell'Oro ${ }^{1,2}$, Giuseppe Mancia ${ }^{2}$, Rita Facchetti ${ }^{2}$, Gino Seravalle $^{3}$, Cesare Cuspidi ${ }^{2}$, Giovanni Corrao ${ }^{2}$, Jennifer Vanoli ${ }^{2}$, Guido Grassi ${ }^{1,2}$

${ }^{1}$ Divisione di Clinica Medica, Ospedale San Gerardo, Monza, Italy, ${ }^{2}$ University of Milano-Bicocca, Milan, Italy, ${ }^{3}$ Istituto Auxologico Italiano- Istituto Scientifico San Luca, Milan, Italy

Introduction: Home and 24-hour blood pressure $\left(\mathrm{BP}_{\mathrm{Home}}\right.$ and $\left.\mathrm{BP}_{24 \mathrm{~h}}\right)$ are believed to improve the prognostic value of office $\mathrm{BP}\left(\mathrm{BP}_{\text {Office }}\right)$ alone, but the evidence has limitations such as that (1) these 3 BPs are characterized by multicollinearity and (2) the procedures adopted do not allow quantification of the prognostic advantage.

Aim: to establish if the addiction of out-of-office to $\mathrm{BP}_{\text {Office }}$ improves prediction of cardiovascular risk.

Methods: One thousand eight hundred thirtythree individuals belonging to the PAMELA (Pressioni Arteriose Monitorate e Loro Associazioni) were followed for 16 years. Prediction of cardiovascular and all-cause mortality was determined via the goodness of fit of individual data (Cox model), the area underlying the receiving operator curves and the net reclassification improvement of cardiovascular and all-cause mortality risk. Calculations were made for $\mathrm{BP}_{\text {Office }}$ alone and after addition of $\mathrm{BP}_{\text {Home }}, \mathrm{BP}_{24 \mathrm{~h}}$, or both, limited to their residual portion which was found to be unexplained by, and thus independent on, $\mathrm{BP}_{\text {Office }}$

Results: With all methods addition of residual out-of-office systolic or diastolic $\mathrm{BP}$ to $\mathrm{BP}_{\text {Office }}$ significantly improved cardiovascular and all-cause mortality prediction. The improvement was more consistent when $\mathrm{BP}_{\text {Home }}$ rather than $\mathrm{BP}_{24 \mathrm{~h}}$ was added to $\mathrm{BP}_{\text {Office }}$ and, compared with $\mathrm{BP}_{\text {Office }}$ plus $\mathrm{BP}_{\text {Home }}$, no better prediction was found when addition was extended to $\mathrm{BP}_{24 \mathrm{~h}}$. With all additions, however, the improvement was quantitatively modest, which was the case also when data were separately analyzed in younger and older individuals or in dippers and nondippers.

Conclusions: Thus, addition of out-of-office to $\mathrm{BP}_{\text {Office }}$ improves prediction of cardiovascular risk, even when data analysis avoids previous limitations. The improvement appears to be limited, however, which raises the question of the advantage to recommend their extended use in clinical practice.

\section{RELATIONSIP BETWEEN UNATTENDED AND ATTENDED BP VALUES AND ARTERIAL STIFFNESS DAMAGE}

Anna Paini, Claudia Agabiti Rosei, Carolina De Ciuceis, Massimo Salvetti, Fabio Bertacchini, Carlo Aggiusti, Deborah Stassaldi, Sara Capellini, Chiara Arnoldi, Marco Cacciatore, Paolo Malerba, Alessandro Maver, Damiano Rizzoni, Maria Lorenza Muiesan.

Internal Medicine, 2 Medicina, University of Brescia \& ASST Spedali Civili di Brescia, Brescia, Italy

Introduction: It has been suggested that measurement of "unattended" or "automated oscillatory (AOBP)" blood pressure values may provide advantages over conventional BP measurement and some hypertension guidelines now suggest this approach as the preferred one for measuring office BP. Data on the relationship between retinal arteriolar alterations and "attended" and "unattended" BP.

AIM: was to evaluate the relationship between "attended" or "unattended" BP values and retinal arteriolar changes in 142 subjects undergoing a visit and assessment of retinal fundus at an ESH Excellence Centre.

MethodS: Both "unattended" BP (patient alone in the room, an oscillometric device programmed to perform $3 \mathrm{BP}$ measurements, at 1 minute intervals, after 5 minutes) and "attended" BP were measured with the same device, on the same day of arterial stiffness assessment, in random order. The wall thickness was measured automatically by a localization algorithm as the difference between External (ED) and internal diameter (ID) was measured by adaptive optics (RTX-1, Imagine Eyes, Orsay, Francia). Media to lumen ratio (WLR) of the retinal arterioles and cross-sectional area (WCSA) of the vascular wall were measured on the basis of vessel diameter and lumen diameter.

Results: Patient's mean age was $57 \pm 12$ yrs, $48 \%$ female, mean BMI $26 \pm 4,60 \%$ had hypertension ( $84 \%$ treated) and $11 \%$ had diabetes mellitus type 2. Systolic unattended BP was lower as compared to attended SBP $(129 \pm 14.8$. vs $122.1 \pm 13.6 \mathrm{mmHg})$. WLR was similarly correlated with systolic unattended BP and with attended SBP $(r=0.281$ e $r=0.382, p<0.0001)$ and with diastolic unattended and attended BP $(\mathrm{r}=0.34$ e $\mathrm{r}=0.29, \mathrm{P}<0.001, \mathrm{p}<$ $0.0001)$. The differences between correlations were not statistically significant (Steiger's Z test).

Conclusion: Measurement of BP "unattended" or "unattended" provides different values, being unattended BP lower as compared to attended BP. Our results suggest that attended and unattended BP values are similarly correlated with retinal arteriolar changes.

\section{OFFICE BLOOD PRESSURE MEASUREMENT: MEAN OF TWO OR OF THREE VALUES?}

Anna Paini, Carlo Aggiusti, Fabio Bertacchini, Deborah Stassaldi, Sara Capellini, Chiara Arnoldi, Roberto Gatta, Giovanni Saccà, Marco Cacciatore, Giulia Arenare, Alessandro Maver, Marzia Santagiuliana, Maria Lorenza Muiesan, Massimo Salvetti

Internal Medicine, University of Brescia and Spedali Civili di Brescia, Brescia, Italy

Introduction: ESC/ESH 2018 hypertension guidelines recommend the calculation of the average of the last 2 blood pressure (BP) measurements (out of 3 ). This recommendation does not strictly correspond to the recommendations of the ESC/ESH guidelines and to those of other international Societies.Furthermore, in several clinical trials mean BP values were calculated in different ways, more frequently by using the mean of three values (out of 3 measurements), or the mean of the last two (out of 2 or of 3 ).

Aim: of the study was to evaluate the effect of different methods for the calculation of the mean value on observed BP values and their relationship with hypertension mediated organ damage.

Methods: In 206 patients BP was measured by a physician with an automated validated oscillometric device ( 3 measurements at $1 \mathrm{~min}$ intervals after 5 minutes of rest).Mean values were computed by using the first two measurements $\left(\mathrm{SAP}_{1 / 2}\right.$ and $\left.\mathrm{DAP}_{1 / 2}\right)$ or the last two $\left(\mathrm{SAP}_{2 /}\right.$ 3 and $\left.\mathrm{DAP}_{2 / 3}\right)$ or three measurements $\left(\mathrm{SAP}_{1 / 2 / 3}\right.$ and $\left.\mathrm{DAP}_{1 / 2 / 3}\right)$.All patients also underwent an echocardiogram.

Results: Mean age was $58 \pm 15$ years, $57 \%$ F, BMI $27 \pm 5,85 \%$ were hypertensives (78\% treated). $\mathrm{BP}$ values were as follows: $\mathrm{SAP}_{1 / 2}$ : $135.5 \pm 17.4 ; \mathrm{SAP}_{1 / 2 / 3}: 134.0 \pm 17.1 ; \mathrm{SAP}_{2 / 3}: 132.4 \pm 16.9 \mathrm{mmHg}$ 
(ANOVA $\mathrm{p}<0.01$ for all comparisons); $\mathrm{DAP}_{1 / 2}: 77.4 \pm 11.0$; $\mathrm{DAP}_{1 / 2 /}$ 3: $77.0 \pm 11.0 ; \mathrm{DAP}_{2 / 3}: 76.7 \pm 11.3$ (ANOVA $\mathrm{p}<0.01$ only for $\mathrm{DAP}_{1 / 2}$ vs $\left.\mathrm{DAP}_{2 / 3}\right)$.Left ventricular mass index $\left(\mathrm{LVMI} / \mathrm{h}^{2.7}\right)$ was correlated with all three means of SAP: $\left(\mathrm{SAP}_{1 / 2}: \mathrm{r}=0.164, \mathrm{p}<\right.$ $0.05 ; \operatorname{SAP}_{1 / 2 / 3}: r=0.168 ; \operatorname{SAP}_{2 / 3}: r=0.185$ ). Despite a trend for a better correlation between $\mathrm{LVMI} / \mathrm{h}^{2.7}$ and $\mathrm{SAP}_{2 / 3}$, no difference between the correlation coefficients was observed at the Steiger's Zeta test.Also the correlations between mean BP values and left atrial volume were similar $\left(\mathrm{SAP}_{1 / 2}: \mathrm{r}=0.174 ; \mathrm{SAP}_{1 / 2 / 3}: \mathrm{r}=0.169 ; \mathrm{SAP}_{2 / 3}: \mathrm{r}=0.170, \mathrm{p}<\right.$ 0.05).Left atrial linear dimensions were correlated only with $\operatorname{SAP}_{2 / 3}(r$ $=0.145, \mathrm{p}<0.05)$.

Conclusions: BP values are lower when the mean of the last two values out of three is calculated as recommended by the $2018 \mathrm{ESC} /$ ESH guidelines. Despite this, the correlation with organ damage seems to be similar.

\section{ASSOCIATION OF CLINIC AND AMBULATORY BLOOD PRESSURE WITH NEW-ONSET ATRIAL FIBRILLATION: A META-ANALYSIS OF OBSERVATIONAL STUDIES}

Francesca Coccina ${ }^{1}$, Anna M. Pierdomenico ${ }^{2}$, Matteo De Rosa ${ }^{1}$, Chiara Cuccurullo $^{2}$, Sante D. Pierdomenico ${ }^{1}$

${ }^{1}$ Department of Innovative Technologies in Medicine \& Dentistry, University "Gabriele d'Annunzio", Chieti-Pescara, Chieti, Italy, ${ }^{2}$ Department of Medicine and Aging Sciences, University "Gabriele d'Annunzio", Chieti-Pescara, Chieti, Italy

Aim: The aim of this study was to perform a meta-analysis of studies evaluating the association of clinic and daytime, nighttime and 24-hour blood pressure (BP) with the occurrence of new-onset atrial fibrillation (AF).

Methods: We conducted a literature search through PubMed, Web of science and Cochrane Library for articles evaluating the occurrence of new-onset $\mathrm{AF}$ in relation to the above-mentioned BP parameters and reporting adjusted hazard ratio and $95 \%$ confidence interval.

Results: We identified five studies. The pooled population consisted of 7224 patients who experienced 444 cases of AF. The overall adjusted hazard ratio (95\% confidence interval) was 1.05 (0.98-1.13), 1.19 (1.11-1.27), 1.18 (1.11-1.26), and 1.23 (1.14-1.32), per 10 -mmHg increment in clinic, daytime, nighttime and 24-hour systolic BP, respectively. The degree of heterogeneity of the hazard ratio estimates across the studies ( $\mathrm{Q}$ and I-squared statistics) were minimal. Conclusions: The results of this meta-analysis suggest that ambulatory systolic BP predicts incident AF better than does clinic systolic $\mathrm{BP}$ and that daytime, nighttime and 24-hour systolic BP are similarly associated with future AF.

\section{CIRCADIAN WATER AND SALT BALANCE AND BLOOD PRESSURE VARIABILITY IN PATIENTS WITH PARKINSON'S DISEASE: EFFECTS OF ANTIHYPERTENSIVE DRUG THERAPY}

Gabriele Busi ${ }^{1}$, Giorgio Mossini ${ }^{1}$, Barbara Palladini ${ }^{1}$, Luigi Gerra ${ }^{1}$, Stefano Tedeschi ${ }^{1}$, Ignazio Verzicco ${ }^{1}$, Anna Calvi ${ }^{1}$, Tomas Macedoni ${ }^{1}$, Riccardo Simoni ${ }^{1}$, Ilaria Fischetti ${ }^{1}$, Stefania Cavazzini ${ }^{2}$, Pietro Coghi ${ }^{1}$, Vanni Vicini ${ }^{1}$, Luisa Musiari ${ }^{1}$, Alberto Montanari ${ }^{1}$, Riccardo Volpi ${ }^{1}$, Aderville Cabassi ${ }^{1}$

${ }^{1}$ Clinica e Terapia Medica, Department of Medicine and Surgery (DIMEC), University of Parma, Parma, Italy ${ }^{2}$ Laboratorio di Tossicologia Industriale, Department of Medicine and Surgery (DIMEC), University of Parma, Parma, Italy

Introduction: Dysautonomia represents an early determinant of the non-motor clinical picture of the patient with Parkinson's disease.

Aim: The aim of the study was to compare the alterations of the water and salt balance in Parkinson disease patients.

Methods: A group of Parkinson disease patients addressing Hypertension Center of our hospital for both hypertensive and hypotensive problems, included in the SHY OR SHAKY study (Supine HYpertension and ORthoStatic Hypotension: A KeY challenge in Parkinson's Disease, a 20-years monocentric observational study on blood pressure variability in these patients with disautonomia) were divided on the basis of the circadian pressure profile (ambulatory BP monitoring for 24 hours) and the clinical picture into: Group 1 (patients with supine hypertension associated with orthostatic hypotension, $\mathrm{n}=11$ ) and Group 2 (normotension nocturnal and orthostatic hypotension, $\mathrm{n}=6$ ).

Results: These patients had day and night diuresis volume monitored as well as urine sodium, potassium creatinine and aquaporin 2 in order to compare alterations in water balance and electroytes control. Group 1 patients were not taking antihypertensive drug therapy when they performed the tests. The subjects of Groups 1 and 2 were compared with healthy control normotensive subjects. Group 1 patients were treated with eprosartan $(200-600 \mathrm{mg} /$ day in the evening, $\mathrm{n}=7)$ or with nicardipine $(10-20 \mathrm{mg}$ in the evening, $\mathrm{n}=4)$ : they repeated ambulatory blood pressure monitoring and urine analysis after 3 weeks. Parkinson's patients had an inverse day/night diuresis volume versus normotensive controls; inverted was also urinary sodium excretion. Nocturnal urinary excretion of aquaporin 2 is also reduced in patients with Parkinson's disease compared to controls. Between Group 1 and 2 (Parkinson's disease patients with different blood pressure profiles) the excretion of aquaporin $2 /$ creatininuria is lower in those with supine hypertension and orthostatic hypotension. Night greater natriuresis and diuresis associated with greater diurnal blood pressure variability in patients with Parkinson's disease and supine hypertension.

Conclusions: The antihypertensive pharmacological treatment resulted in an improvement in the nocturnal pressure profile, albeit with differences in terms of the hydrosaline balance and the finding of diurnal hypotension episodes. The use of nicardipine induced a greater nocturnal natriuresis which could affect a greater frequency of diurnal orthostatic hypotension. 


\section{Kidney}

\section{ACUTE KIDNEY INFARCTION IN A 50 YEARS OLD MAN: FROM AN ACUTE PYELONEPHRITIS TO A SYSTEMIC ARTERIAL UNCOMMON DISEASE-FIBROMUSCULAR DYSPLASIA}

Pappaccogli Marco ${ }^{1}$, Licata Carolina ${ }^{2}$, Fanelli Elvira ${ }^{1}$, Eula Elisabetta $^{1}$, Novelli Giulia ${ }^{1}$, Savio Daniele ${ }^{3}$, Veglio Franco ${ }^{1}$, Rabbia Franco $^{1}$

${ }^{1}$ Division of Internal Medicine and Hypertension Unit, Department of Medical Sciences, University of Turin, Turin, Italy, ${ }^{2}$ Department of Nephrology and Dialysis, Ciriè, Italy, ${ }^{3}$ Interventional RadiologyNeuroradiology Department, SG Bosco Hospital, Turin, Italy

Introduction: Fibromuscular Dysplasia (FMD) is an idiopathic, segmental, non-inflammatory and non-atherosclerotic disease of the musculature of arterial walls, leading to stenosis of small- and medium-sized arteries. Although gender related FMD distinctive features remain to be further investigated, men seem to be rarely affected from the disease and more likely to present with arterial dissection and focal stenosis.

Aim: We describe the case of a young male with multivessel and multifocal FMD presenting with acute renal infarction.

Methods: A 50 years old men was admitted to the emergency department suffering from fever and right flank pain without dysuria or stranguria. All investigations performed (urine test and culture, serum C-reactive protein, ultrasound and contrast-enhanced CT of the abdomen) were negative and the patient was discharged with the diagnosis of right renal colic.

Two days later, the patient was re-admitted to the emergency department for symptoms persistence. A new contrast-enhanced CT, showing a wide area of poor parenchymal enhancement in the medium and lower third of the right kidney, was performed and the patient was then recovered in the Nephrology Unit with the suspicion of acute pyelonephritis.

During the hospitalization, the diagnostic tests (urine test and culture, serum C-reactive protein and creatinine levels) were persistently negative and no response to antibiotic therapy with ceftriaxone and levofloxacin was observed. After a careful revision of the CT scan by an interventional radiologist and the results of a magnetic resonance and of a duplex ultrasound (US), the diagnosis of right mesorenal infarction due to an arterial thrombosis with occlusion of the corresponding lobar branch of the right renal artery in the context of a small arterial dissection was established. The overall CT imaging revision lead also to the diagnosis of a coeliac trunk ectasia and a 3 centimeter-length string of beads lesion of the splenic artery with multiple severe stenosis, highly suspicious for multifocal FMD.

Patient's evaluation was completed with a cerebrovascular CT angiography showing an intracranial sacciform aneurysm of the middle cerebral artery (MCA), compatible with intracranial involvement of FMD. Screening for inherited or acquired thrombophilia were negative and 24-hour blood pressure (BP) values were normal (BP $118 / 66 \mathrm{mmHg}$ )

Results: A diagnosis of multivessel FMD with visceral (multifocal stenosis of splenic artery), renal (arterial dissection complicated with kidney infarction) and intracranial (sacciform aneurysm) involvement in a middle-aged smoking man was made.

After one year from the diagnosis, renal scintigraphy resulted normal, vascular FMD-related lesions remained unchanged at a subsequent CT-scan.

Conclusions: FMD is an under-recognized cause of renovascular hypertension, often affecting young or middle-aged women, and described as an idiopathic, segmental, non-inflammatory and nonatherosclerotic disease of the musculature of arterial walls, leading to stenosis of small- and medium-sized arteries FMD phenotype also includes aneurysms, arterial dissections and arterial tortuosity.

\section{THE GLOMERULAR HYPERFILTRATION IS ASSOCIATED TO ARTERIAL STIFFNESS AND CENTRAL HEMODYNAMIC STRESS IN HYPERTENSIVE PATIENTS INDEPENDENTLY BLOOD PRESSURE VALUES}

Pietro Nazzaro, Adele Nardecchia, Maria Contini, Gianfranco Amodio, Gabriella Schirosi, Lorenzo Debenedittis, Gabriella Aceto, Serena Calò, Mariangela Lisena

Dept. Biomedical Sciences and Human Oncology, Hypertension Unit "AM. Pirrelli", University of Bari "Aldo Moro", Bari, Italy

Introduction: Several studies have shown, in different diseases such as hypertension, the glomerular overfiltration as preclinical marker, but very few have investigated the association of renal hyperfiltration with functional vascular damage.

Aim: To identify the role of higher glomerular filtration rate (eGFR) as marker of impaired arterial function in treated hypertensives

Methods: We studied 510 grade- 1 hypertensives, treated by ACEi o ARB as monotherapy, with similar hypertensive state, confirmed by ABPM, divided by standardized eGFR range, in order of sex and age (NKF's-KDOQI), in 71 with definitively impaired values (RED), 134 with values within the lower half (NLO) and 187 within the higher half (NUP) of the appropriate normal range and, finally, 118 with a glomerular hyperfiltration rate (HYP). The patients, after a medical visit (off), underwent tonometry to measure arterial stiffness (PWVcf), augmentation index (AIx) and central pulse pressure (PPcar).

Results: Patients presented similar smoke habit, BMI (29, $4 \pm 3,9$ vs $29,4 \pm 5,2$ vs $30,4 \pm 5,3$ vs $29,5 \pm 5,7$ ) and microalbuminuria (ACR: $28,9 \pm 23,3$ vs $26,5 \pm 23,7$ vs $26,6 \pm 33,8$ vs $26,8 \pm 34$, 2) but also other significant characteristics. $(\mathrm{m} \pm$ s.d.: *: $\mathrm{p}<.05$, **: $\mathrm{p}$ $<.01, * * *: \mathrm{p}<.001$ vs RED; ${ }^{\wedge}: \mathrm{p}<.05, \wedge \wedge: \mathrm{p}<.01,{ }^{\wedge \wedge \wedge}: \mathrm{p}<.001$ vs NLO; ${ }^{\circ}: \mathrm{p}<.05,{ }^{\circ}: \mathrm{p}<.01,{ }^{\circ}: \mathrm{p}<.001$ vs NUP).

\begin{tabular}{|c|c|c|c|c|c|}
\hline pts/var & eGFR & SBP/DBPoff & PWVcf & Alx & PPcar \\
\hline RED & $65 \pm 14$ & $133 \pm 14 / 80 \pm 7$ & $12.1 \pm 3.9$ & $14.9 \pm 11.7$ & $45.8 \pm 15.1$ \\
\hline NLO & $88 \pm 16 * * *$ & $133 \pm 13 / 81 \pm 9$ & $9.6 \pm 2.7 * * *$ & $12.1 \pm 13.9$ & $45.3 \pm 13.2$ \\
\hline NUP & $94 \pm 10 * * * \wedge \wedge \wedge$ & $135 \pm 14 / 81 \pm 9$ & $9.5 \pm 2.4 * * *$ & $13.8 \pm 12.6$ & $42.5 \pm 11.4$ \\
\hline HYP & $98 \pm 12^{* * * \wedge \wedge \wedge \wedge^{\circ}}$ & $136 \pm 16 / 79 \pm 8$ & $11.9 \pm 3.6^{\wedge \wedge \wedge} \wedge^{\circ 00}$ & $18.1 \pm 12.6^{\wedge \wedge \wedge^{\circ}}$ & $50.2 \pm 13.2 * \wedge \wedge^{\circ 00}$ \\
\hline
\end{tabular}

HYP patients, althought showed a similar SBP/DBPoff, demonstrated higher arterial stiffness, similarly to the RED but additionally, a higher hemodynamic central arterial strain. 
Conclusions: The findings showed that the glomerular hyperfiltration may represent an event associated, even in treated hypertensives, to impaired peripheral arterial compliance and enhancement of the pulse wave volume with pathophysiological consequences that should be deeply investigated.

\section{INTRADIALYTIC HYPERTENSION: A CASE REPORT}

Turrin Giada, Rivasi Giulia, D’Andria Maria Flora, Tortu' Virginia, Capacci Marco, Rapi Michele, Ungar Andrea

Referral Centre for Hypertension Management in Older Adults, Division of Geriatric and Intensive Care Medicine, Careggi Hospital and University of Florence, Italy

Introduction: in some patients blood pressure (BP) increases during dialysis (systolic BP $>180 \mathrm{mmHg}$ ); this phenomenon is known as "intradialytic hypertension".

Aim: We describe a case of intradilytic hypertension.

Methods: case report

Results: B.F., 73 years, affected by chronic kidney disease requiring dialysis. He referred to the Hypertension Clinic of Careggi Hospital, Florence, Italy, for intradialytic hypertension. The patient was receiving antihypertensive treatment with ramipril, oral clonidine and furosemide. During dialysis he was treated with iv clonidine, with no benefit on intradialytic BP. Home BP monitoring showed optimal BP control (mean BP 130-135/80 mmHg). Ambulatory BP monitoring confirmed appropriate daytime BP control associated with a reverse dipping profile, thus raising the suspicion of cardiovascular dysautonomia. Office BP measurements revealed orthostatic hypotension. Based on available literature, use of low-sodium and low-calcium dialysate and increased fluid removal during dialysis were suggested to reduce fluid overload and cardiac inotropism. Moreover, treatment with low-dose captopril before dialysis was suggested. Given the outof-office BP values and hypotensive risk, titration of antihypertensive therapy was not recommended. Improved of intradialytic BP control was observed during the following months.

Conclusions: "intradialytic hypertension" is reported in $5-15 \%$ of dialysis patients and is likely related to volume overload, increased vascular resistance, adrenergic and renin-angiotensin system (RAS) activation, electrolyte imbalances and removal of antihypertensive drugs. Management strategies include increased fluid removal during dialysis (enhanced-frequency and extended-time treatment), use of low-sodium dialysate and administration of RAS inhibitors or other non-dialyzed antihypertensive drugs. In our patient, we cannot exclude that intradialytic hypertension also derived from white coat effect and supine hypertension in the context of cardiovascular dysautonomia. As the prognostic relevance of supine hypertension is unclear to date, we estimated that hypotensive risk deriving from orthostatic hypotension and dysautonomia prevailed on potential benefits of a more intensive BP control and we decided not to potentiate interdialytic antihypertensive therapy
Anti-Hypertensive Therapy and Corona Virus Disease 19

\section{PROTECTIVE ROLE OF CARDIOVASCULAR THERAPY IN THE OLDEST ADULTS HOSPITALIZED FOR COVID-19: ANGIOTENSIN RECEPTOR BLOCKERS ARE ASSOCIATED WITH LESS IN-HOSPITAL MORTALITY}

Francesco Spannella $^{1,2}$, Federico ${ }^{1,2}$, Caterina Garbuglia ${ }^{1,2}$, Chiara Di Pentima $^{1,2}$, Valentina Bordoni ${ }^{1,2}$, Andrea Filipponi ${ }^{1,2}$, Sara Falzetti ${ }^{1,2}$, Piero Giordano $^{1}$, Riccardo Sarzani ${ }^{1,2}$

${ }^{1}$ Internal Medicine and Geriatrics, IRCCS INRCA, Ancona, Italy, ${ }^{2}$ Department of Clinical and Molecular Sciences, University "Politecnica delle Marche", Ancona, Italy

Introduction: Older population has a greater risk of morbidity and mortality in Covid-19. Previous cardiovascular diseases represent a risk factor for severe Covid-19 and death. Inhibitors of the reninangiotensin-aldosterone system (RAASi) and statins may have a protective role on mortality in the older adults.

Aim: The aim of the study was to evaluate the association between RAASi/statin therapy and in-hospital mortality in the oldest adults affected by Covid-19.

Methods: We conducted an observational study on oldest patients (age $\geq 80$ years) affected by Covid- 19 and consecutively hospitalized at IRCCS INRCA (Ancona) from March 2020 to February 2021. Patients with chronic bed-rest syndrome in the terminal phase of disease were excluded. The SARS-CoV-2 infection was confirmed with standard molecular test on nasal/oro-pharyngeal swab (rtPCR). Medical history, home and in-hospital cardiovascular therapy and laboratory parameters at admission were analyzed as possible predictors of in-hospital mortality.

Results: We enrolled 337 patients with a mean age of $87.5 \pm 6.1$ years and a female prevalence of $56.1 \%$. The $76.1 \%$ of patients had a documented history of hypertension and the $46.6 \%$ had a concomitant heart failure at admission (diagnostic criterion: NT-proBNP $\geq 1800$ $\mathrm{pg} / \mathrm{ml}$ ). In-hospital mortality was $43.9 \%$. There was no association between mortality and home therapy with RAASi $(\mathrm{p}=0.219)$ or statin $(\mathrm{p}=0.137)$. Inversely, patients treated with angiotensin receptor blockers (ARBs) during hospitalization had a lower mortality than untreated patients $(32.8 \%$ vs $53.5 \%, \mathrm{p}<0.001)$. This association was independent of $\mathrm{O} 2$ or C-PAP requirement and remained significant even after adjustment for age, sex, dementia, albumin, eGFR, NTproBNP levels, leukocytes [OR: 0.3 (95\% CI 0.2-0,6), $\mathrm{p}<0.001$ ].

Conclusions: Therapy with ARBs during hospitalization is associated with lower in-hospital mortality in oldest patients hospitalized for Covid-19, probably due to the protective role on the cardiovascular and pulmonary systems exerted by the "acute " modulation of the RAAS. Our data confirm the benefit of ARBs even in the multimorbid oldest population. 


\section{CORONA VIRUS DISEASE 19 (COVID-19) EFFECT ON BLOOD PRESSURE CONTROL IN HYPERTENSIVES}

\author{
Sebastiano Cicco ${ }^{1,3}$, Chiara Calvanese ${ }^{2}$, Maria Rosaria Bellotta ${ }^{3}$, \\ Pierangelo Tedesco ${ }^{2}$, Angelo Vacca ${ }^{1,3}$, Immacolata Panettieri ${ }^{2}$, \\ Giuseppe Ranieri ${ }^{3}$
}

${ }^{1}$ COVID section, Unit of Internal Medicine "Guido Baccelli", Department of Biomedical Sciences and Human Oncology, University of Bari Aldo Moro, Bari, Italy; ${ }^{2}$ Centro Ipertensione Arteriosa, UOC Medicina Interna Ospedaliera, AUO Ospedali Riuniti, Foggia, Italy; ${ }^{3}$ Centro Ipertensione, Unit of Internal Medicine "Guido Baccelli", Department of Biomedical Sciences and Human Oncology, University of Bari Aldo Moro Medical School, Bari, Italy

Introduction: SARS-CoV-2 virus Angiotensin Converting Enzyme 2 (ACE2) to enter target cells. ACE2 is one of the main molecular pathways in arterial hypertension in general population. Cardiovascular diseases and especially arterial hypertension are some of the main comorbidities in COVID patients.

Aim: Aim of this study is to verify if SARS-CoV-2 infection may induce a change in blood pressure control in patients already treated for hypertension.

Methods: We evaluated patients came to office follow-up for blood pressure between January 1st and March 31st 2021 who had history of COVID-19 (Group 1, $7 \mathrm{~F}$ and $11 \mathrm{M}$, aged $65.22 \pm 8.22$ ) in previous six months confirmed by molecular PCR analysis on nasal swab. As control group, we selected a paired number of patients matched for comorbidities, age and sex (Group 2, $7 \mathrm{~F}$ and $11 \mathrm{M}$, aged $64.73 \pm$ 9.73). We collected in-office systolic (SBP) and Diastolic (DBP) blood pressure and the medication taken at the time of visit and we compared them to same values collected in the visit performed in 2020 and before SARS-CoV-2 infection. We also compared BMI to evaluate a possible influence of body mass change on blood pressure. Results: No differences were found in DBP nor BMI between the two groups and comparing 2021 visit to 2020 in each group. Group 1 shows significant increase of SBP compared to Group 2 (128.4 \pm 14.1 vs $120.2 \pm 16.4 \mathrm{mmHg}, \mathrm{p}<0.05)$ at the enrolment. Group 1 shows also a statistical tendency to a significant increase of 2021 SBP values compared to $2020(128.4 \pm 14.1$ vs $123.1 \pm 12.5 \mathrm{mmHg}, \mathrm{p}=0.08)$. On the contrary, Group 2 had a significant decrease in 2021 SBP values compared to 2020 (120.2 \pm 16.4 vs $126.7 \pm 10.1 \mathrm{mmHg}, \mathrm{p}<$ $0.05)$. We also found a significant increase of the number of medication used in 2021 by Group 1 compared to $2020(1.9 \pm 0.9$ vs $2.2 \pm$ $1.1 ; \mathrm{p}<0.05)$, as well as an increase the number of medication taken in 2021 by Group 1 patients compared to Group $2(1,6 \pm 0,9$ vs 2,2 $\pm 1,1 ; \mathrm{p}<0.05)$. No differences were found in Group 2 between the two visit point and between the two Groups in 2020

Conclusions: In-office patients who experienced COVID-19 presents a worsening in blood pressure control after infective disease remission.

\section{ANGIOTENSIN II UPREGULATES ACE-2 EXPRESSION VIA AT1 RECEPTOR IN HUMAN BRONCHIAL EPITHELIAL CELLS}

Ilaria Caputo, Brasilina Caroccia, Teresa M. Seccia, Gian Paolo Rossi

Specialized Center for Blood Pressure Disorders-Regione Veneto and Internal Emergency Medicine Unit, Department of Medicine-DIMED, University of Padua, Padua, Italy
Introduction: The Angiotensin (Ang) converting enzyme (ACE)-2 is the cellular receptor of SARS-CoV-2 and ACE inhibitors (ACEIs) and Ang type 1 receptor (AT1R) antagonists (ARBs) were contended to raise its expression, thus facilitating the SARS-CoV-2 infection but no evidence supports this hypothesis.

Aim: We investigated the effect of Angiotensin (Ang) II, ACE inhibitors (ACEIs) and Ang type 1 receptor (AT1R) antagonists (ARBs) on ACE-2 mRNA and protein levels in human epithelial bronchial cells.

Methods: Calu-3 cells, bronchial epithelial cells derived from lung adenocarcinoma, were treated with $10 \mu \mathrm{M}$ irbesartan (ARB), $10 \mu \mathrm{M}$ ramipril (ACEi) or $10 \mu \mathrm{M}$ MLN-4760 alone or on top of $100 \mathrm{nM}$ Ang II. ACE-2 mRNA expression was evaluated by Digital droplet (dd)PCR after 12 hours treatment, instead ACE-2 protein levels were evaluated by Western Blot after 48 hours treatment.

Results: We showed that Ang II increased the mRNA ( $p=0,0007)$ and protein $(\mathrm{p}=0,03)$ levels of ACE-2, while the ACEI ramipril and the ARB irbesartan did not. The effect of Ang II on ACE-2 was AT1R-mediated, because it was abolished by irbesartan $(p=0,007)$ and unaffected by ramipril. Blockade of ACE-2-mediated conversion of Ang II into Ang- (1-7) with MLN-4760 did not affect the Ang IIinduced ACE-2 increase.

Conclusions: Therefore, as Ang II upregulates ACE-2 in human bronchial cells by acting through the AT1R, blockade of Ang II formation and/or action can lower the SARS-CoV-2 receptor and thus the spread of the COVID-19 in patients with activated renin-angiotensin-aldosterone system.

\section{BLOOD PRESSURE AND LIPID PROFILE IN HYPERTENSIVE PATIENTS POST THE FIRST COVID-19 LOCKDOWN}

\section{ILARIA FUCILE, MARIA VIRGINIA MANZI, RAFFAELE IZZO, NICOLA DE LUCA, VALENTINA RUSSO, COSTANTINO MANCUSI}

Hypertension Research Center and Department of Advanced Biomedical Science, Federico II University Hospital, Naples, Italy

Introduction: In Italy the lockdown was imposed in March 2020 to contrast and contain the spread of the new COVID-19. During lockdown people were forced to stay in their homes which has resulted in change in physical activity, dietary pattern and psychological status of the individuals.

Aim: The main objective of this study is to evaluate the impact of this constraint on blood pressure control, body weight, lipid profile and fasting glucose in hypertensive patients.

Methods: This observational study included 72 hypertensive patients visited at Hypertension Research Center of Federico II University Hospital in Naples between May and July 2020. BMI, lipid profile, blood glucose and office blood pressure were collected for subsequent comparison with last available visit before lockdown date.

Results: After $1^{\text {st }}$ lockdown period no significant changes in Body weight, lipid profile and blood glucose were found compared to previous visits while a significant reduction in systolic and diastolic values was observed $(139 \pm 17.55 \mathrm{VS} 132 \pm 20.45)(78 \pm 9.59 \mathrm{VS}$ $75 \pm 11.57)(\mathrm{p}<0.05)$.

Conclusions: The pandemic induced some challenging lifestyle habits, many of these that were changed favorably during quarantine are well-known modifiable risk factors for hypertension and might be associated with the observed reduction of BP. 
Therapy, Pharmacoeconomy and Pharmacosurveillance

\section{PERMANENT PACEMAKER IMPLANTATION FOLLOWING SEVERE ELECTROLYTES DISTURBANCES DURING LENALIDOMIDE TREATMENT IN HYPERTENSIVE PATIENT WITH MULTIPLE MYELOMA}

Alice Bresolin ${ }^{1}$, Antonella Paola Sacco ${ }^{2}$, Silvia Rizzati ${ }^{2}$, Pietro Guerra $^{1}$, Gioia Torin ${ }^{2}$, Stefano Cuppini ${ }^{2}$, Francesco Zanon ${ }^{3}$, Alberto Mazza $^{4}$

${ }^{1}$ Internal Medicine Unit, Santa Maria della Misericordia General Hospital, Rovigo (Italy) and School of Internal Medicine University of Padua. Padua, Italy, ${ }^{2}$ Department of Internal Medicine, Santa Maria della Misericordia General Hospital, Rovigo, Italy, ${ }^{3}$ Arrhythmia and Electrophysiology Unit, Division of Cardiology, Santa Maria Della Misericordia General Hospital, Rovigo, Italy, ${ }^{4}$ Unit of Hypertension and ESH Excellence Hypertension Centre, Department of Internal medicine, Santa Maria della Misericordia General Hospital, Rovigo, Italy

Introduction: In patients with multiple myeloma (MM) the development of cardiovascular diseases may derive from factors related to MM treatment as the use of antineoplastic drugs.

Aim: The cardiovascular (CV) adverse events due to treatment with lenalidomide (LDM), an immunomodulatory drug used in the treatment of MM refractory to conventional therapy, are described below. Methods: A 75-years-old man was hospitalized in the Internal Medicine Unit (January 2021) for sudden onset of confusion, agitation, paresthesias of the lips and extremities and sphinteric release with non-contrast cerebral TC negative for ischemic/hemorrhagic events. He was affected by MM IgG lambda from 2 years, in oral treatment from February 2020 with $25 \mathrm{mg}$ LDM once-daily three weeks monthly and $20 \mathrm{mg}$ dexamethasone weekly. He reported arterial hypertension, hyper-cholesterolemia, mild carotid atherosclerotic disease, stage- 3 chronic kidney disease and pulmonary embolism due to deep vein thrombosis occurred 8 months earlier, for which he was taking oral therapy with perindopril/amlodipine $5 / 5 \mathrm{mg} /$ daily, atorvastatin $40 \mathrm{mg} /$ daily, rivaroxaban $20 \mathrm{mg} /$ daily, oxycodone/naloxone 20/10mg/daily, aciclovir 400mg and lansoprazole 30mg/daily. The blood pressure (BP) levels were uncontrolled (i.e. $160 / 96 \mathrm{mmHg}$ ) and then normalized with increasing the dose of perindopril/amlodipine up to $10 / 10 \mathrm{mg} /$ daily.

Results: Laboratory tests showed severe total/ionized hypocalcaemia $(6,59 / 3,35 \mathrm{mg} / \mathrm{dl}$ respectively), moderate hypokalaemia $(2.68 \mathrm{mmol} /$ L), normal serum albumin and magnesium, mild vitamin-D deficiency $(11,2 \mathrm{ng} / \mathrm{mL})$, high serum creatinine levels $(1,34 \mathrm{mg} / \mathrm{dl})$ with normal phosphatemia. The electrocardiogram (ECG) revealed a sinus rhythm with right bundle branch block, left anterior hemiblock (already present in a previous ECG), intermittent second degree Mobitz type II atrio-ventricular block and QTc prolongation $(508 \mathrm{msec})$. After discontinuation of therapy with LDM/dexamethasone and the intravenous administration of calcium $(9 \mathrm{~g}) /$ potassium $(80 \mathrm{mEq})$ neurological symptoms disappeared, but despite the resolution of electrolytes disturbances, an ECG-Holter confirmed trifascicular block (Figure) and the patient underwent to permanent dual-chamber pace-maker implantation.

Conclusions: In patients with MM hypertension is relatively common $(38 \%)$ and often difficult to control. Hypocalcaemia and hypokalaemia are very rare side effect of LDM/dexamethasone therapy, that increase the risk of thromboembolic events and cardiac arrhythmias. In hypertensive patients with $\mathrm{MM}$, in the presence of electrocardiographic abnormalities, an interdisciplinary collaboration (cardiologist/internist/oncologist) is mandatory to prevent early and late CV side effects.

\section{Bundle branch block (A) and second degree Mobitz type II atrio-ventricular block (B), non-conducted $\mathrm{P}$ waves (arrows), not followed by QRS complex and not preceded by interval PR prolongation.}

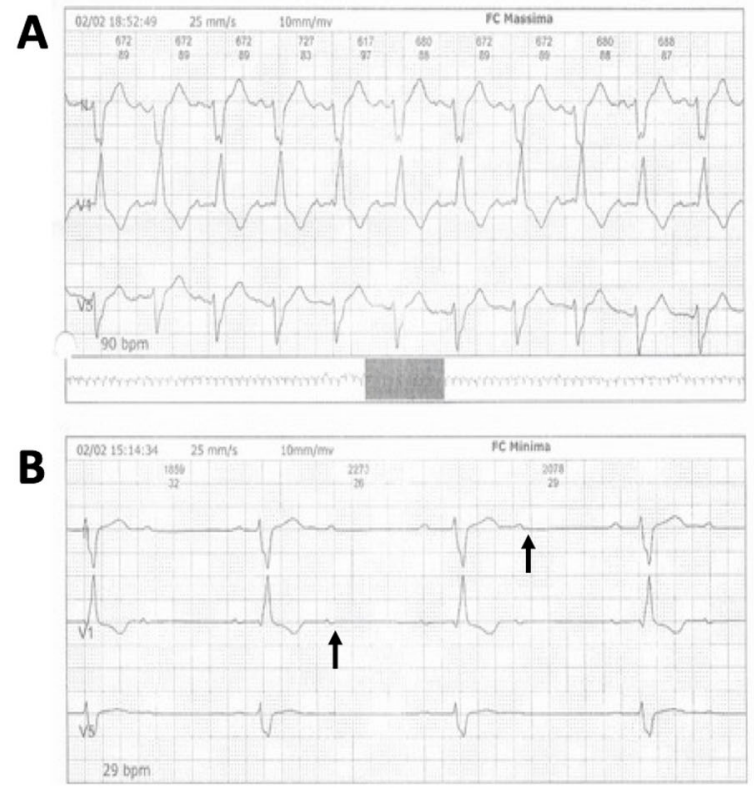




\section{SERIAL MEASUREMENT OF ALDOSTERONE-TO- RENIN RATIO FOR THE ASSESSMENT OF ANTI- HYPERTENSIVE DRUG ADHERENCE}

Fabrizio Buffolo ${ }^{1}$, Elisa Sconfienza ${ }^{1}$, Jacopo Burrello ${ }^{1}$, Isabel Losano $^{1}$, Giulio Mengozzi ${ }^{2}$, Gabriella Priolo ${ }^{2}$, Valeria Avataneo ${ }^{3}$, Antonio D'Avolio ${ }^{3}$, Franco Veglio ${ }^{1}$, Franco Rabbia ${ }^{1}$, Paolo Mulatero $^{1}$, Silvia Monticone ${ }^{1}$

${ }^{1}$ Division of Internal Medicine and Hypertension Unit, Department of Medical Sciences, University of Turin Turin, Italy, ${ }^{2}$ Department of Laboratory Medicine, AOU Città della Salute e della Scienza, Turin, Italy, ${ }^{3}$ Department of Medical Sciences, University of Turin, Laboratory of Clinical Pharmacology and Pharmacogenetics, Amedeo di Savoia Hospital, Turin, Italy

Introduction: Achievement of blood pressure control is hampered by several factors, including partial or absent compliance to prescribed medications. The available methods for the assessment of therapeutic adherence are characterized by low accuracy, high costs and/or limited applicability in clinical practice.

Aim: We designed a prospective study to assess the therapeutic compliance to renin-angiotensin-aldosterone system (RAAS) inhibitors by serial measurement of aldosterone-to-direct renin ratio (ARR). Methods: We prospectively recruited 80 patients: 40 subjects with arterial hypertension and 40 normotensive controls. In patients with arterial hypertension, blood samples were collected at baseline and 2 and 8 weeks after initiation of a RAAS inhibitor for ARR determination. In the control group, ARR was evaluated at baseline and at 2 weeks. Compliance to anti-hypertensive treatment was confirmed by therapeutic drug monitoring.

Results: Direct renin significantly increased and aldosterone significantly decreased after initiation of RAAS inhibitor, with reduction of ARR. We defined delta ARR ( $\triangle \mathrm{ARR})$ as the relative change in ARR before and after treatment initiation. $\triangle \mathrm{ARR}$ provided high accuracy for determination of therapeutic adherence, with an area under the curve (AUC) of 0.900 at 2 weeks and 0.886 at 8 weeks. We selected a cut-off with maximized sensitivity, guaranteeing a minimum specificity of $75 \%$. A cut-off of $-48 \%$ of $\triangle$ ARR provided $90 \%$ sensitivity and $75 \%$ specificity, both at 2 and 8 weeks.

Conclusions: Measurement of $\triangle \mathrm{ARR}$ is an accurate test, cheap and widely available that allows determination of non-adherence to RAAS inhibitors prescription. We therefore propose the implementation of $\triangle \mathrm{ARR}$ in clinical practice through a multi-step flow-chart for the management of patients with uncontrolled hypertension, that allows the identification of subjects suspected to be non-adherent, reserving therapeutic drug monitoring for non-adherence confirmation.

\section{MULTIPLAT_AGE: A MULTICOMPONENT INTERVENTION PLATFORM FOR MANAGING COMMUNITY DWELLING OLDER SUBJECTS WITH MULTIMORBIDITY AND POLYPHARMACY}

\author{
Alberto Pilotto $^{1,2,3}$, Guido Iaccarino ${ }^{4}$, Armando Genazzani ${ }^{5}$, Carlo \\ Trompetto $^{6}$, Gennarina Arabia ${ }^{7}$, Paolo Tonin ${ }^{8}$, Sabrina Zora ${ }^{1,2}$, Erica \\ Volta $^{1,2}$, Maddalena Illario ${ }^{4}$, Vincenzo De Luca ${ }^{4}$, Laura Mori ${ }^{6}$, Loris \\ Pignolo $^{8}$, Marianna Contrada ${ }^{8}$, Alessandra Argusti ${ }^{1}$, Alberto Ferri ${ }^{1,2}$, \\ Alberto Cella ${ }^{1,2}$ \\ ${ }^{1}$ Multiplat_Age Research Unit, E.O. Galliera Hospital, Genova, Italy, \\ ${ }^{2}$ Department Geriatric Care, Orthogeriatrics and Rehabilitation, \\ E.O. Galliera Hospital, Genova, Italy, ${ }^{3}$ Department of
}

Interdisciplinary Medicine, University of Bari "Aldo Moro", Bari, Italy, ${ }^{4}$ Federico II University, Centro Interdipartimentale di Ricerca in Ipertensione Arteriosa e Patologie Correlate, Napoli, Italy, ${ }^{5}$ A.O.U. Maggiore della Carità, Novara, e Università del Piemonte Orientale, Novara, ${ }^{6}$ UOC Neurorehabilitation Unit, Department of Neuroscience, University of Genova, Genova, Italy, ${ }^{7}$ Department of Neurology, A.O.U. Mater Domini, Università "Magna Grecia", Catanzaro, Italy, ${ }^{8}$ Istituto Sant'Anna, Crotone, Italy

Introduction: Multimorbid elderly subjects represent a heterogeneous population at high risk for negative health oucomes. Multicomponent tailored interventions may prevent functional and cognitive impairments. However, implementing these strategies of care is often limited by the fragmented delivery of care, causing unappropriate use of care services and increase in health-related costs. Aim: MULTIPLAT_AGE is a co-funded project by the Italian Ministry of Health aiming at implementing an ICT-based platform of common strategies to improve the management of elderly with multimorbidity and polypharmacy.

Methods: The Project is based on five work-packages developing innovative research in five different hospitals in four Italian regions: i) Galliera Hospital (Genova-Liguria): a pilot study aims to test a homecare protected area able to host subjects and their caregivers after discharge from the hospital; ii) Federico II University (NapoliCampania): an ICT-based home-care program was implemented to: a) identify the determinants of negative events and b) to evaluate how ICT tools may support caregivers to assist patients (e.g. therapy adherence); iii) Maggiore della Carità Hospital (Novara-Piemonte): a study on appropriate pharmacological treatments (particularly anticoagulant therapy) in primary-care and in the Emergency Department was designed; iv) San Martino Hospital (Genova-Liguria): a study on the efficacy of technology rehabilitation to improve motor ability in older healthy people and neurological patients; v) Sant'Anna Hospital and Magna Grecia University (Crotone/Catanzaro-Calabria): a pilot study is ongoing on using cognitive stimulation in a telerehabilitation setting for Parkinson' disease and post-stroke patients with mild cognitive impairment.

Results: The final expected results of the project will include: a) development and validation of tailored solutions of care management and b) improvement in functional and cognitive parameters of the study populations.

Conclusions: The MULTIPLAT_AGE project network will develop and validate multicomponent intervention strategies to improve the health-care management of older people with multimorbidity and polypharmacy.

\section{ANALYSIS OF DRUGS AND METABOLITES IN HAIR AS AN OBJECTIVE TOOL FOR MONITORING ADHERENCE TO ANTIHYPERTENSIVE AND LIPID LOWERING THERAPY. AN ANALYTICAL CHALLENGE}

Rossella Gottardo, Francesco Taus, Marco Ballotari, Pietro Minuz, Franco Tagliaro

\section{University of Verona, Verona, Italy}

Introduction: A poor adherence to therapy in chronic treatments has been reported in several pathological conditions including hypertension and dyslipidemias. This impacts severely public health and pharmaco-economy. Unfortunately, the monitoring of adherence to therapy using traditional approaches based on questionnaires and/or blood and urine testing proves highly inaccurate (see "Adherence to Long-Term Therapies, Evidence for Action", WHO, 2003, request of 
copies: adherence@who.int). On the other hand, hair analysis for drugs and metabolites, although widely used in forensic toxicology to monitor drug abuse, has only occasionally been proposed in this context. Indeed, the keratinized tissues incorporate at the root level the xenobiotics present in capillary blood and, lacking any metabolism in the hair stalk, protect these molecules for long time. In short, these features make hair an almost ideal specimen for monitoring long term exposure to drugs. On the other hand, hair analysis, because of the limited amounts of sample which can be collected and the low drug incorporation rate, poses important problems of sensitivity and, collaterally, selectivity, which only modern chromatography/mass spectrometry can overcome.

Aim: Aim of the present work was to verify the suitability of liquid chromatography hyphenated with mass spectrometry to quantitatively determine drugs and metabolites in the hair of subjects undergoing chronic treatments with statins and antihypertensive drugs (betablockers, calcium antagonists, ACE inhibitors).

Methods: Blank hair for preparation of quality controls and calibration standards were obtained from well-known subjects abstinent from any medicinal drugs for the previous 6 months. Hair samples (n $=50$ ) were collected from authentic users under therapy with statins and antihypertensive drugs. The hair was cut into small fragments, incubated overnight under acidic conditions and then extracted with SPE. In alternative, the fragmented hair was sonicated with methanol at room temperature. UHPLC-QqQ-MS/MS was applied for the quantitative determination of the analytes.

Results: The developed methods were fully validated according to international guidelines, showing excellent sensitivity and selectivity with negligible matrix effects and very good linearity, accuracy, precision and extraction recovery. Overall, in the "positive" hair the concentrations of atenolol ranged from 0.42 to $3.99 \mathrm{ng} / \mathrm{mg}$, for amlodipine from "trace" to $35.4 \mathrm{ng} / \mathrm{mg}$, for bisoprolol from "traces" to $26.7 \mathrm{ng} / \mathrm{mg}$. The most prescribed statins were rosuvastatin and atorvastatin which were detected in hair at concentration ranging from 0.22 to $79.2 \mathrm{ng} / \mathrm{mg}$. In some cases, also drug metabolites could be detected.

Conclusions: The preliminary results show, on one hand, a real possibility of providing accurate determinations of drugs in hair from subjects following standard therapies for hypertension and dyslipidemic conditions and, on the other hand, pose sound grounds for the adoption of this approach to objectively monitor adherence to long term pharmacological treatments

\section{SEVERE POLYCYTHEMIA DUE TO AXITINIB THERAPY IN METASTATIC RENAL CELL CANCINOMA: A RARE CASE OF SECONDARY ARTERIAL HYPERTENSION}

Ettore Mancia, Maria Giovanna Vario, Dario Altieri, Santina Cottone

ESH Hypertension Excellence Centre and Nephrology Unit, Department of Health Promotion, Mother and Child Care, Internal, University of Palermo, Palermo, Italy

Introduction: Hypertension and higher haemoglobin levels are the most common adverse effects of Vascular Endothelial Growth Factorinhibitors (VEGF-inhibitors), that are associated with longer survival in patients with metastatic renal cell carcinoma (mRCC).

Aim: We report a rare case of severe polycythaemia due to Axitinib therapy in patient with $\mathrm{mRCC}$, that required phlebotomies and discontinuation therapy.

METHODS: A 60-year-old man with a history of mRCC underwent right radical nephrectomy in 2016. After initial therapy with
Sunitinib, the patient received Axitinib $10 \mathrm{mg} /$ daily for four years and repeated chest CT scan showed no progression of metastatic lung disease.

The patient came to our Centre for severe hypertension that was confirmed by $24 \mathrm{~h}$-ABPM. Abdominal ultrasound showed enlarged left kidney $(14 \mathrm{~cm})$. On Doppler duplex examination, there was no renal artery stenosis and renal resistive index (IRR) values were normal $($ IRR $=0.61)$. Ophthalmoscopic examination showed arteriovenous crossings.

Screening for primary aldosteronism was negative (aldosterone 90 $\mathrm{ng} / \mathrm{L}$, renin $11 \mathrm{ng} / \mathrm{L}$ and aldosterone to renin ratio 8.88). Blood laboratory tests showed severe polycythaemia ( $\mathrm{Hb} 20.6 \mathrm{~g} / \mathrm{dl}$, RBCs $7200000 / \mathrm{mm} 3$, Htc $61.6 \%$ ). The erythropoietin levels were $12.8 \mathrm{mIU} /$ $\mathrm{mL}$ (normal range 3-20).

Results: The patient required three phlebotomies followed by reduction of haemoglobin $(\mathrm{Hb} 13 \mathrm{~g} / \mathrm{dl})$ and of blood pressure values. Axitinib therapy was promptly suspended and treatment with Nivolumab was started.

Conclusions: Inhibition of VEGF signalling could induce hepatic synthesis of erythropoietin through HIF- $1 \alpha$ independent mechanism. Moreover, it increases peripheral arteriolar resistance by reducing nitric oxide and prostacyclin synthesis, enhancing ET-1 production and inducing micro-vessel rarefaction. We suggest the importance of strict control of blood pressure and haemoglobin values in patient treated by VEGFr-inhibitors.

\section{Vessels and Endothelium}

\section{URIC ACID IN CHRONIC CORONARY SYNDROMES: RELATIONSHIP WITH CORONARY ARTERY DISEASE SEVERITY AND LEFT VENTRICULAR DIASTOLIC PARAMETER}

Massimiliano Monticelli ${ }^{1,2}$, Alessandro Maloberti ${ }^{1,2}$, Filippo Leidi $^{1,2}$, Irene Bossi ${ }^{3}$, Elena Tassistro ${ }^{4}$, Paola Rebora ${ }^{4}$, Angelo Racioppi ${ }^{2}$, Stefano $\mathrm{Nava}^{3}$, Francesco Soriano ${ }^{3}$, Emanuele Piccaluga ${ }^{3}$, Giacomo Piccalo $^{3}$, Jacopo Oreglia ${ }^{3}$, Roberto Pirola ${ }^{1}$, Benedetta De Chiara ${ }^{1}$, Fabrizio Oliva ${ }^{3}$, Antonella Moreo ${ }^{1}$, Maria Grazia Valsecchi ${ }^{4}$ and Cristina Giannattasio ${ }^{1,2}$

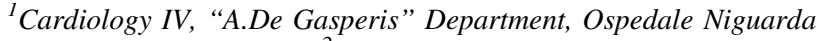
Ca' Granda, Milan, Italy, ${ }^{2}$ School of Medicine and Surgery, MilanoBicocca University, Milan, Italy, ${ }^{3}$ Cardiology I, "A.De Gasperis" Department, Ospedale Niguarda Ca' Granda, Milan, Italy, ${ }^{4}$ Bicocca Center of Bioinformatics, Biostatistics and Bioimaging (B4 center), School of Medicine and Surgery, University of Milano-Bicocca, Monza, Italy

Introduction: Uric Acid (UA) has been related to the development of Cardio-Vascular (CV) events in patients affected by Chronic Coronary Syndromes (CCS). Among various hypothesis, two arise: UA may negatively act on coronary artery determining a higher degree of atherosclerotic disease, and/or on heart determining a higher prevalence of diastolic dysfunction.

Aim: aim of our investigation was to evaluated the relatioship between UA and coronary artery atherosclerotic disease and diastolic dysfunction in CCS patients.

Methods: 231 patients who were admitted to the cardiological department of the Niguarda Hospital (Milan, Italy) for CCS from January 2017 to June 2018 were enrolled. Coronary atherosclerotic burden was evaluated from coronary angiography as the number and type of involved vessels, as well as with both Gensini and Syntax scores. All subjects underwent a complete echocardiogram. 
Results: At unadjusted and adjusted/multivariable analysis, UA levels were not significantly associated with variables analyzed from the coronary angiography (number and type of vessels involved, neither the Gensini and Syntax scores) as well as with echocardiographic parameters regarding systolic and diastolic function.

Conclusions: the main finding of our work is the absence of a role for UA in determining coronary arteries disease as well as LV diastolic dysfunction in CCS subjects. Taking together the results of previous studies with ours, we hypothesize that UA could act on heart (both on coronary arteries and on LV function) in an early phase of the disease, whereas while in the advanced stages other factors (previous myocardial infarction, previous myocardial revascularization and so on) may overshadow its effects.

\section{MITRAL VALVE REPLACEMENT FOR NON- INFECTIVE ENDOCARDITIS IN A YOUNG HYPERTENSIVE SUBJECT WITH MOYAMOYA SYNDROME: SEARCHING FOR A LINK}

Pietro Guerra ${ }^{1}$, Alice Bresolin ${ }^{1}$, Antonella Paola Sacco ${ }^{2}$, Daniela Lanza $^{3}$, Luca Conte ${ }^{3}$, Paolo Scaramuzzo ${ }^{4}$, Giuseppe Martire ${ }^{5}$, Fabio Dell'Avvocata $^{6}$, Giuseppe Petrilli ${ }^{7}$, Claudio Picariello ${ }^{8}$, Enrico Orvieto $^{9}$, Ciro Rossetti ${ }^{2}$, Laura Schiavon ${ }^{2}$, Gioia Torin ${ }^{2}$, Marta Milan $^{2}$, Stefano Cuppini ${ }^{2}$, Alberto Mazza ${ }^{10}$

${ }^{1}$ Internal Medicine Unit, Santa Maria della Misericordia General Hospital, Rovigo (Italy) and School of Internal Medicine University of Padua, Padua, Italy, ${ }^{2}$ Department of Internal Medicine, Santa Maria della Misericordia General Hospital, Rovigo, Italy, ${ }_{3}$ Unit of Cardiology, Santa Maria Della Misericordia General Hospital, Rovigo, Italy, ${ }^{4}$ Unit of Nephrology, Santa Maria Della Misericordia General Hospital, Rovigo, Italy, ${ }^{5}$ Unit of Radiology, Santa Maria Della Misericordia General Hospital, Rovigo, Italy, ${ }^{6}$ Unit of Cardiovascular and Endoluminal Interventions, Santa Maria Della Misericordia Hospital, Rovigo, Italy, ${ }^{7}$ Division of Cardiac Surgery, University of Verona, Verona, Italy, ${ }^{8}$ Unit of Intensive Care Cardiology, Unit of Cardiology, Santa Maria Della Misericordia Hospital, Rovigo, Italy, ${ }^{9}$ Pathology Unit, Santa Maria Della Misericordia Hospital, Rovigo, Italy, ${ }^{10}$ Unit of Hypertension and ESH Excellence Hypertension Centre, Department of Internal medicine, Santa Maria della Misericordia General Hospital, Rovigo, Italy

Introduction: Moyamoya disease (MMD) is a rare cerebrovascular disorder characterized by a progressive steno-occlusion of the internal carotid artery and an abnormal vascular network of the cerebral arteries. An extensive basal telangiectasia collateral circulation develops, which has the cerebral angiographic appearance of a "puff of smoke", in Japanese moya-moya. MMD has a bi-modal incidence with an early peak before age 15 and a second peak in the $3 \mathrm{rd}$ and 4 th decade. The clinical picture is characterized by recurrent, transient neurologic ischemic events and minor strokes, but intracerebral haemorrhage can also occur, and additional atherosclerotic lesions can be found in the adult. Patients with the characteristic MMD who also have well-recognized associated conditions are categorized as having the Moyamoya syndrome (MMS).

Aim: We report a case of non-infective endocarditis in a young hypertensive patient with occlusion of the internal carotid artery and with brain imaging suggestive for MMS.

METHODS: A 48-year-old woman presented to the emergency department for worsening dyspnea and increased blood pressure levels (i.e. $160 / 90 \mathrm{mmHg}$ ) without chest pain. The patient had a history of hypertension for 15 years, diagnosed after a minor stroke and for which she was taking oral antihypertensive therapy with amlodipine $10 \mathrm{mg}$ and acetylsalicylic acid $100 \mathrm{mg}$ once daily. She reported two skin ulcers on the legs that had arisen 2 years earlier, on which she applied an aqueous solution of boric acid; she also referred alcohol consumption and active cigarette smoking. At the examination patient not had a fever but she had agitated and at time hyperactive with not very fluent speech. She had clinical features of heart failure and pulmonary oedema and the cardiac auscultation revealed normal first and second heart sounds, a grade 4/6 pansystolic murmur at the apex along with a long diastolic murmur.

Results: The chest X-ray showed pulmonary congestion and bilateral pleural effusion and the electrocardiogram revealed sinus tachycardia (115 bpm), bi-atrial enlargement and signs of left ventricular hypertrophy without repolarization abnormalities. The laboratory tests showed mild normocytic anemia (hemoglobin $11.5 \mathrm{~g} / \mathrm{l}$ ), neutrophilic leucocytosis $(10.430 / 13.22 \mathrm{~mm} 3)$, increase in C-reactive protein (4.51 $\mathrm{mg} / \mathrm{dl})$, D-Dimer $(2.94 \mathrm{mcg} / \mathrm{ml})$, N-terminal pro-brain natriuretic peptide $(5856 \mathrm{pg} / \mathrm{ml})$, serum creatinine $(2.23 \mathrm{mg} / \mathrm{dl})$, proteinuria (1628 mg/24h), parathyroid hormone $(285 \mathrm{pg} / \mathrm{ml})$, serum glycaemia $(160 \mathrm{mg} / \mathrm{dl})$ and glycated-hemoglobin $(7 \%)$; partial thromboplastin time, serum troponin-I and pro-calcitonin were within normal limits ( $36 \mathrm{sec}, 31 \mathrm{ng} / \mathrm{L}$ and $0.15 \mathrm{ng} / \mathrm{ml}$, respectively). Lung perfusion singlephoton emission tomography (SPECT) using [99mTc]-labelledmacroaggregated albumin excluded pulmonary embolism but revealed a pulmonary thickening at the base of the right lung. Transthoracic echocardiography revealed normal left ventricular function (ejection fraction of $67 \%$ without kinetic alterations) but the mitral valve showed thickened flaps and with a cloth-like formation that enveloped both flaps with frayed edges leading to moderate stenosis and moderate-severe insufficiency; there was also slight enlargement of the left atrium (volume $60 \mathrm{ml} / \mathrm{mq}$ ) and increased in pulmonary artery systolic pressure $(55 \mathrm{mmHg})$. A sub-sequent transesophageal echocardiogram confirmed the presence of mitral valve with thickened and hyper-echogenic flaps, slightly hypomobile due to the presence of two coarse vegetations adhering to both valve flaps with irregular, normo-echogenic, very mobile edges, measuring $1.3 \times 0.7$ and $1.4 \mathrm{x} 1 \mathrm{~cm}$ respectively. According to the European Society of Cardiology three sets of blood cultures collected from different venipuncture sites, with at least $1 \mathrm{~h}$ between the first and last draw were performed. Broad-spectrum antibiotic therapy was then started with piperacillin-tazobactam and the inflammation indices quickly normalized. Serologies for M. Pneumoniae, C. Pneumoniae, Bartonella and Coxiella, markers for HIV, HBV and HCV infection, T. Pallidum, urinary antigens for L. Pneumophila and S. Pneumoniae, galactomannan and beta-D-glucan were normal. The autoimmune tests (ANCA, ENA, ANA, LAC and antiphospholipid antibodies) were negative. The PET-CT there was neither increase in the $18 \mathrm{~F}$ FDG uptake in the cardiac valve, nor in other body districts, which made difficult a marantic aetiology of valvulopathy. Doppler of renal arteries was normal, while ultrasound showed signs of hypertensivebased medical nephropathy (probably due to several cardiovascular risk factors of our patient), confirmed by renal biopsy that excluded vasculitis. We also investigated the anamnestic finding of the juvenile stroke performing a carotid duplex ultrasound that revealed the occlusion of the internal right carotid artery. The contrast-enhanced cerebral computed tomography allowed the identification of a polydistrict vasculopathy with total occlusion of the right internal carotid, a $50 \%$ stenosis of the left subclavian artery, and a Moyamoya pattern of the intracranial circulation with the absence of the posterior communicating artery and the presence of different collateral circles perfusing the cerebral media artery (Figure 1, A and B). The patient underwent mitral valve replacement surgery with a Hancock $29 \mathrm{~mm}$ biological prosthesis and left auricle closure with a $45 \mathrm{~mm}$ Atriclip (Figure 2, A and B). The histological section of the mitral valve resulted devoid of inflammation and the culture of the native valve not identified microbial agents. 
Conclusions: Although an association between MMD and renovascular hypertension has been known, the literature on hypertension without renal artery stenosis is scarce. The pathogenesis of nonbacterial endocarditis is not entirely known, but involve endothelial cell dysfunction in a setting of hypercoagulable state, like in hypertension and MMD. Finally, the association between MMD and cardiovascular diseases derives from clinical cases and is proposed in the cardio-cephalic syndrome of the neural crest $(*)$. If such evidence is anecdotal or there is a potential physio-pathological association of this triad, targeted studies are needed.

*) Komiyama M. Interv Neuroradiol 2017; 23: 572-576.

(Figure 1, And B)

\section{TARGETING SIRT1 RESCUES AGING AND OBESITY-INDUCED MICROVASCULAR DYSFUNCTION IN HUMANS}

Alessandro Mengozzi ${ }^{1,2}$, Sarah Costantino ${ }^{3}$, Francesco Paneni ${ }^{3,4,5}$, Emiliano Duranti ${ }^{1}$, Nicola Riccardo Pugliese ${ }^{1}$, Stefano Taddei ${ }^{1}$, Agostino Virdis ${ }^{1}$, Stefano Masi ${ }^{1,6}$

\footnotetext{
${ }^{1}$ Department of Clinical and Experimental Medicine, University of Pisa, Pisa, Italy, ${ }^{2}$ Institute of Life Sciences, Scuola Superiore Sant'Anna, Pisa, Italy, ${ }^{3}$ Center for Molecular Cardiology, University of Zürich, Zürich, Switzerland, ${ }^{4}$ Department of Cardiology, University Heart Center, University Hospital Zurich, Zürich, Switzerland, ${ }^{5}$ Department of Research and Education, University Hospital Zurich, Zürich, Switzerland, ${ }^{6}$ Institute of Cardiovascular Science, University College London, London, United Kingdom
}

Figura 1. A) Angio-TC cerebrale assiale can occlusione del sifone carotideo destro (1) e del tratto M1 prossimale destro [2]. Eslli e tortuosi circoll collaterall provenienti dall'arteria comunicante anteriore con aspetto a anuwola di fumo* (3) riforniscono larteria cerebrale media destra (MCA) che è di calibro ridotto (4) rispetto alla controlaterale. B) Ricostruzione volume Rendering di Anglo-TC cerebrale, con piccoll circoli collaterall tortuosi allorigine e nel tratto M1 prossimale della MCA destra (5) con aspetto a "nuwola di fumon, esile il tratto M1 distale, M2 ed M3 di destra (6), assente opacizzazione del sifone carotideo destro e del tratto A1 destro (7), aspetto pluri-stenotico di ramo fronto-insulare di $M 2$ destro (Q) con ipertrofia dell'arteria ricorrente di Heuber ( 9 ).
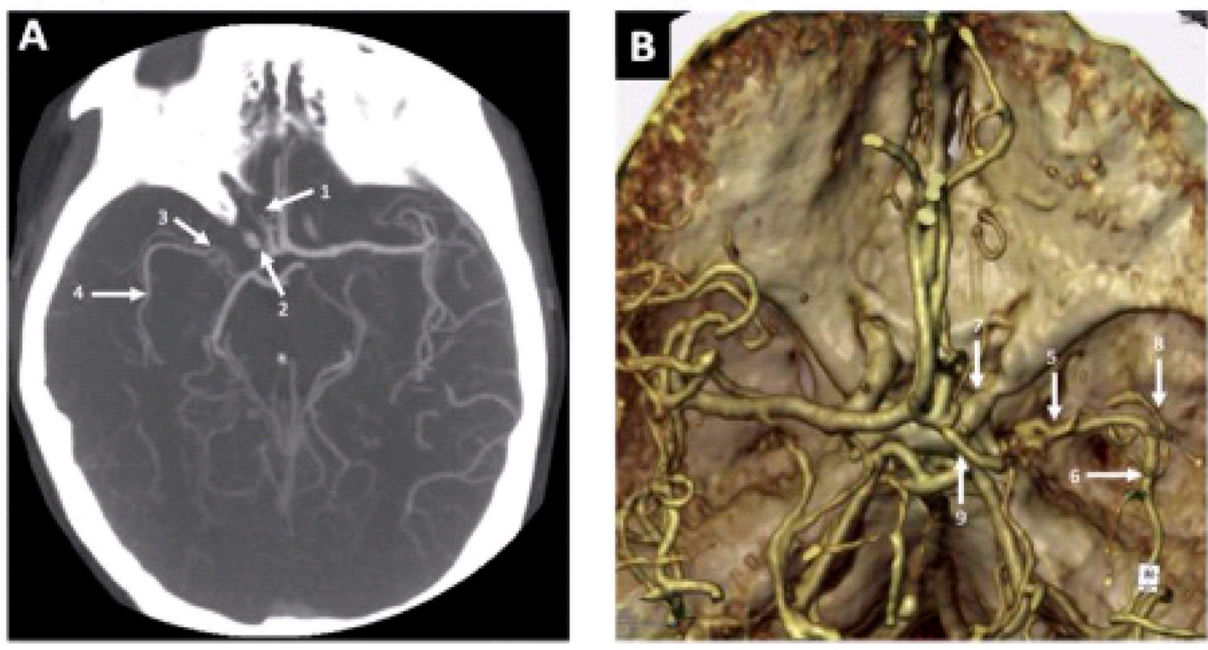

Figura 2. A) Visione intra-operatoria della valvola mitrale prima dell'escissione.

B) Lembo posteriore della valvola mitrale sezionato in 2 parti, con presenza di abbondanti fenomeni di deposizione fibrinosa su valvola con apparente degenerazione sclero-calcifica.
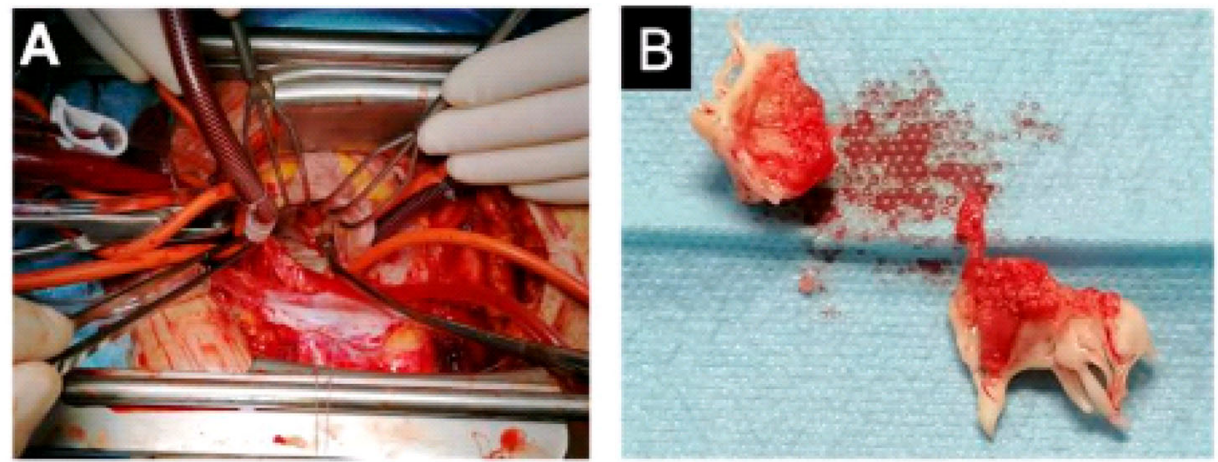
Introduction: Experimental evidence suggests a key role of SIRT1 in the age and metabolic-related vascular dysfunction. Whether these effects hold true in the human vasculature is unknown.

Aim: To investigate the role of SIRT1 in the age- and obesity-related microvascular dysfunction in humans.

Methods: Forty-seven obese and forty-eight control subjects undergoing elective laparoscopic surgery were recruited and stratified based on their age (above or below 40 years) in 4 groups: young obese, young control, old obese and old control. Endothelial function was assessed in small resistance arteries by pressurised micromyography, before and after incubation with a SIRT1 agonist (SRT1720) and a mitochondria reactive oxygen species scavenger (MitoTEMPO). Vascular production of mitochondria reactive oxygen species and nitric oxide availability were assessed by confocal microscopy, while vascular gene expression of SIRT1 and mitochondrial proteins was assessed by qPCR. ChIP assay was employed to investigate SIRT1-dependent epigenetic regulation of mitochondrial proteins.

Results: Obese patients showed lower expression of SIRT1 and mitochondrial antioxidant proteins (FOXO3 and SOD2), and a higher expression of pro-oxidant and aging mitochondria proteins $\mathrm{p} 66^{\mathrm{Shc}}$ and Arginase II. Old obese, young obese and old control groups displayed an impairment of endothelial function which was rescued by SRT1720 and MitoTEMPO (Figure). SRT1720 incubation reduced mitochondria reactive oxygen species and improved nitric oxide availability in old obese arteries. These effects were explained by SIRT1-dependent chromatin changes leading to reduced $\mathrm{p}^{\text {Shc }}$ expression and upregulation of proteins involved in mitochondria respiratory chain.

Conclusions: Therapeutic modulation of SIRT1 restores obesity and age-related endothelial dysfunction through an epigenetic control on several mitochondrial pathways.

\section{FIGURE}

\section{D ORGAN CULTURE LONG-TERM STUDY TO REPRODUCE THE IMMUNE-VASCULAR INTERFACE BETWEEN RESISTANCE ARTERIES AND IMMUNE CELLS, ESTABLISHED BY HYPERTENSION}

Lorenzo Carnevale $^{1}$, Fabio Pallante ${ }^{1}$, Sara Perrotta $^{2}$, Marialuisa Perrotta $^{2}$, Daniela Carnevale ${ }^{1,2}$, Giuseppe Lembo ${ }^{1,2}$

${ }^{1}$ Research Unit of Neuro and Cardiovascular Pathophysiology, IRCC Neuromed, Department of Angiocardioneurology and Translational Medicine, Pozzilli (IS), Italy, ${ }^{2}$ Department of Molecular Medicine, "Sapienza" University of Rome, Rome, Italy

Introduction: The chronic exposure of resistance arteries to high blood pressure is a determinant factor in the typical remodeling, which overall contributes to sustain blood pressure increase by enhancing the peripheral resistances. Immune cells recruitment to the vascular district is a critical step in this process. However, the mechanisms of this immune-vascular interaction are largely unknown, making it difficult designing therapeutical interventions targeting this interface. To overcome these limitations, it is necessary to develop a reliable and physiologically relevant experimental model of the immune-vascular interface.

Aim: With this work we show the possibility to implement a threedimensional vascular culture system in pressure myography that recapitulates the in-vivo resistance arteries properties.

Methods: The first setup step for the system was the viability study to ensure the endothelial and muscular function of the cultured vessel up to 72 hours from excision. After a daily check of vessel contractility and viability, we tested at $72 \mathrm{~h}$ endothelial function and myogenic tone, which resulted equal to function of a acutely studied
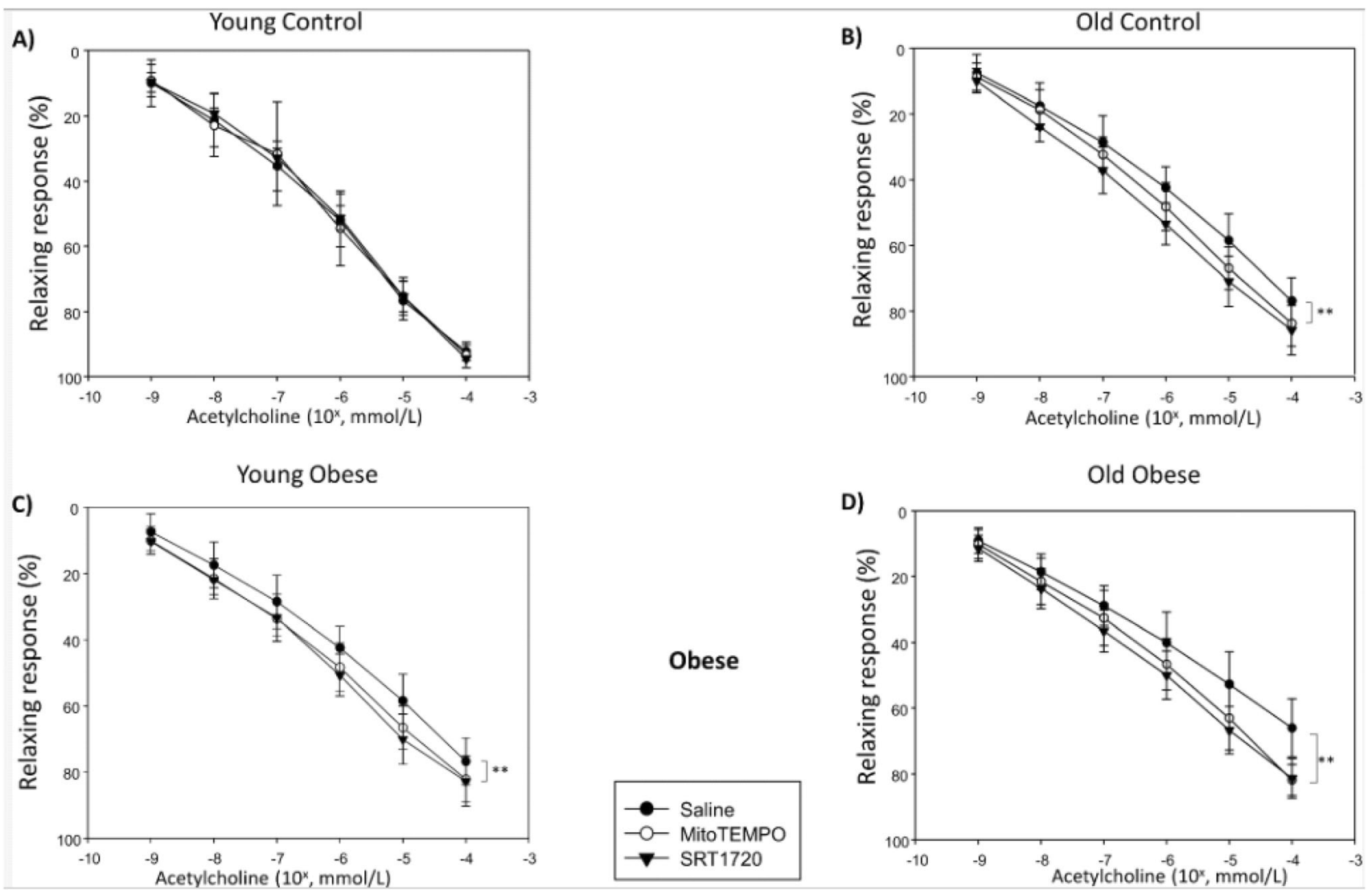
vessel. Using this technological platform, we co-cultured immune cells of interest to reproduce ex-vivo the hypertensive immune-vascular interface.

Results: In the 3D model we demonstrate that CD8 T cells extracted from hypertensive mice after Angiotensin II infusion increment the contractile capabilities of resistance arteries excised from naïve normotensive mice, while the same treatment with CD4 from hypertensive mice did not result in altered myogenic tone.

Conclusions: These finding suggest that CD8 positive cells directly contribute to the increment of peripheral resistance in hypertension.

\section{A NOVEL VASOACTIVE PENTAPEPTIDE "PG1" FROM BUFFALO ICE-CREAM PROTECTS FROM ANGIOTENSIN-EVOKED HIGH BLOOD PRESSURE}

Albino Carrizzo $^{1,2}$, Paola di Pietro ${ }^{1}$, Manuela Giovanna Basilicata ${ }^{3}$, Giacomo Pepe ${ }^{3}$, Antonio Damato ${ }^{2}$, Pietro Campiglia ${ }^{3}$, Carmine Vecchione ${ }^{1,3}$

${ }^{I}$ Department of Medicine and Surgery, University of Salerno, Baronissi, SA, Italy, ${ }^{2}$ IRCCS Neuromed, Vascular pathophysiology Unit, 86077, Pozzilli (IS), Italy, ${ }^{3}$ Department of Pharmacy, University of Salerno, Fisciano, SA, Italy

Introduction: Arterial hypertension is the most important risk factor for cardiovascular diseases, myocardial infarction, heart failure, renal failure and peripheral vascular disease. In the last decade, milkderived bioactive peptides have attracted attention for their beneficial cardiovascular properties.

Aim: Identify new vasoactive natural peptides able to fight arterial hypertension that can be used as a preventive strategy.

Methods: Here we combined in-vitro chemical assay such as LC-MS/ MS analysis of buffalo ice cream, ex-vivo vascular studies evaluating endothelial and smooth muscle responses using pressure myograph, and translational assay testing in-vivo the vascular actions of PG1 administration in murine models.

RESULTS: We demonstrate that a novel buffalo ice-cream-derived pentapeptide "QKEPM", namely PG1, is a stable peptide that can be obtained at higher concentration after gastro-intestinal digestions (GID) of buffalo ice-cream (BIC). It owns potent vascular effect in counteract the effects of angiotensin II-evoked vasoconstriction and high blood pressure levels. Its effects are mediated by the inhibitory effect on AT1 receptor leading to a downregulation of $\mathrm{p}-\mathrm{ERK}^{1} \mathrm{1} / 2$ Rac1-GTP and consequent reduction of oxidative stress.

Conclusions: These results strongly candidate PG1, as a novel bioactive peptide for the prevention and management of hypertension, thus expanding the armamentarium of preventive strategies aimed at reducing the incidence and progression of hypertension and its related cardiovascular complicications.
ACCURACY OF A NEW INSTRUMENT FOR NONINVASIVE EVALUATION OF PULSE WAVE VELOCITY: THE ATHOS (ARTERIAL STIFFNESS FAITHFUL TOOL ASSESSMENT) PROJECT

Dario Leone $^{1}$, Irene Buraioli ${ }^{2}$, Giulia Mingrone ${ }^{1}$, Davide Lena ${ }^{3}$, Alessandro Sanginario ${ }^{2}$, Fabrizio Vallelonga ${ }^{1}$, Francesco Tosello ${ }^{1}$, Eleonora Avenatti ${ }^{1}$, Marco Cesareo ${ }^{1}$, Anna Astarita ${ }^{1}$, Lorenzo Airale $^{1}$, Luca Sabia ${ }^{1}$, Franco Veglio ${ }^{1}$, Danilo Demarchi ${ }^{2}$, Alberto Milan $^{1}$

${ }^{1}$ Department of Medical Sciences, Division of Internal Medicine, Hypertension Unit, AO Città della Salute e della Scienza di Torino, University of Turin, Turin, Italy, ${ }^{2}$ Department of Electronics and Telecomunications, Politecnico di Torino, Turin, Italy, ${ }^{3} S T$ Microelectronics

Introduction: Large artery stiffness, assessed by carotid-femoral Pulse Wave Velocity (cfPWV), is a major risk factor for cardiovascular events, commonly used for risk stratification. Currently, the reference device for non-invasive cfPWV is SphygmoCor, but its cost and technically challenging use limit its diffusion in clinical practice. Aim: To validate a new device for non-invasive assessment of cfPWV, ATHOS (Arterial sTiffness faitHful tOol aSsessment), designed in collaboration with the Politecnico di Torino, against the reference non invasive method represented by SphygmoCor.

Methods: 90 healthy subjects were recruited. In each subject, we assessed cfPWV, using SphygmoCor $\left(\mathrm{PWV}_{\text {SphygmoCor }}\right)$ and ATHOS $\left(\mathrm{PWV}_{\mathrm{ATHOS}}\right)$ devices in an alternate fashion, following the ARTERY Society guidelines. The accuracy was assessed by Bland-Altman plot, and reproducibility was assessed by inter-operator correlation coefficient (ICC).

Results: Mean $\mathrm{PWV}_{\mathrm{ATHOS}}$ and mean $\mathrm{PWV}_{\text {SphygmoCor }}$ were $7.88 \pm$ $1.96 \mathrm{~m} / \mathrm{s}$ and $7.72 \pm 1.95 \mathrm{~m} / \mathrm{s}$, respectively. Mean difference between devices was $0.15 \pm 0.56 \mathrm{~m} / \mathrm{s}$, with a high correlation between measurements $(\mathrm{r}=0.959, \mathrm{p}<0.001$, figure 1$)$. Considering only PWV values $\geq 8 \mathrm{~m} / \mathrm{s}(\mathrm{n}=30)$, mean difference was $0.1 \pm 0.63 \mathrm{~m} / \mathrm{s}$. The ICC was $97.7 \%$ with ATHOS.

Conclusions: ATHOS showed an excellent level of agreement with SphygmoCor, even at high PWV values, with a good reproducibility. Its simplicity of use could help increase clinical application of PWV assessment, improving patients' cardiovascular risk stratification.
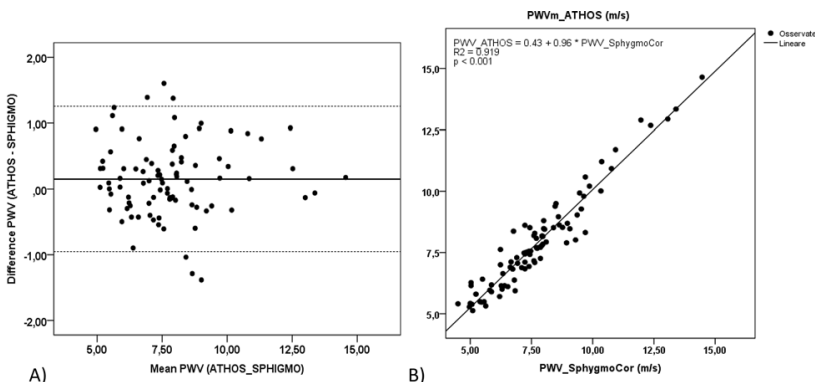

Figure 1. Comparison between PWV values measured with SphygmoCor and the new device ATHOS. A) Blant Altman diagram. B) Scatter plot with linear regression (continuous line). 